\title{
Conformal properties in classical minimal surface theory
}

\author{
William H. Meeks III and Joaquín Pérez
}

\begin{abstract}
This is a survey of recent developments in the classical theory of minimal surfaces in $\mathbb{R}^{3}$ with an emphasis on the conformal properties of these surfaces such as recurrence and parabolicity. We cover the maximum principle at infinity for properly immersed minimal surfaces in $\mathbb{R}^{3}$ and some new results on harmonic functions as they relate to the classical theory. We define and demonstrate the usefulness of universal superharmonic functions. We present the compactness and regularity theory of Colding and Minicozzi for limits of sequences of simply connected minimal surfaces and its application by Meeks and Rosenberg in their proof of the uniqueness of the helicoid. Finally, we discuss some recent deep results on the topology and on the index of the stability operator of properly embedded minimal surfaces and give an application of the classical Shiffman Jacobi function to the classification of minimal surfaces of genus zero.
\end{abstract}

\section{Contents}

1. Introduction.

2. Basic results in classical minimal surface theory.

3. Conformal questions on minimal surfaces.

4. Stable minimal surfaces.

5. The Ordering Theorem for the ends of properly embedded minimal surfaces.

6. Quadratic area growth and recurrence.

7. Maximum principle at infinity for properly immersed minimal surfaces. 304

8. Flux conjectures and some properties of harmonic functions in parabolic Riemannian manifolds.

2000 Mathematics Subject Classification. Primary 53A10, Secondary 49Q05, 53C42.

Key words and phrases. Minimal surface, conformal structure, harmonic function, recurrence, parabolicity, harmonic measure, universal superharmonic function, Jacobi function, stability, index of stability, curvature estimates, maximum principle at infinity, limit tangent plane at infinity, minimal lamination, locally simply connected, blow-up on the scale of topology, parking garage, minimal planar domain.

Research of the first author was partially supported by NSF grant DMS - 0104044 and NSF DMS 9803206.

Research of the second author was partially supported by a MCYT/FEDER grant no. BFM2001-3318. 
9. The Colding-Minicozzi curvature estimates, compactness and regularity

of limit laminations and applications.

10. Topological aspects of the theory of minimal surfaces.

11. The Shiffman Jacobi function on properly embedded planar domains. 327 References

\section{Introduction.}

In this report on recent developments in the classical theory of minimal surfaces, we will focus on some of the spectacular progress that the subject has experienced in the past decade. The theme here is to explore relationships between the function theory and the conformal structure of complete embedded minimal surfaces. Embeddedness influences both local and global properties of these surfaces. This is because embeddedness allows one to use the minimal surface as a barrier against itself to construct stable minimal surfaces in its two complements in $\mathbb{R}^{3}$, see Subsection 4.2. These stable minimal surfaces act as guide posts for deciphering the structure and geometry of the surfaces and their complements.

The recent ground breaking work by Colding and Minicozzi in $[\mathbf{2 4}, \mathbf{1 8}, \mathbf{1 7}, \mathbf{1 9}$, $\mathbf{2 3}, \mathbf{1 4}]$ has been especially influential. Their theorems have been essential for recent progress made in understanding the local and global structure of embedded minimal surfaces which are simply connected or which have finite genus. Their results on the compactness and (partial) regularity of limits of sequences of uniformly locally simply connected minimal surfaces in Riemannian three-manifolds is a major story which we will only briefly touch on here; we refer the reader to our forthcoming survey $[\mathbf{7 3}]$ for a more complete discussion on the Colding-Minicozzi theory and its applications.

We now briefly outline the material of the survey. In Section 2 we cover most of the basic results in minimal surface theory. This Section offers a quick introduction to the main definitions, examples and classical results, and should provide the needed background to beginners in the subject. In Section 3 we go into some of the recent advances on the conformal structure of minimal surfaces with boundary. Section 4 is devoted to the important classical results on stable minimal surfaces. We include here a proof of the beautiful estimate of Colding-Minicozzi on the area of a stable minimal disk, a result on which we base the proofs of the other main theorems of the Section. In Section 5 we study properly embedded minimal surfaces with more than one end and discuss the basic ordering theorem of the ends of such surfaces. In Section 6 we explain how universal superharmonic functions (a concept developed in Section 3) can be used to obtain quadratic area growth estimates for the middle ends of properly embedded minimal surfaces, a result which implies that such surfaces can have at most two limit ends; this is a theorem of Collin, Kusner, Meeks and Rosenberg [26]. Another important application of universal superharmonic functions in this Section shows that when the minimal surface has exactly two limit ends, then it is recurrent for Brownian motion. In Section 7 we outline the recent proof of the general maximum principle at infinity for properly immersed minimal surfaces by Meeks and Rosenberg. Previous maximum principles at infinity have had a unifying effect on the theory and this final version will likely play a similar important role. A deep application of this principle appears in the proof 
that a properly embedded minimal surface in $\mathbb{R}^{3}$ with absolute Gaussian curvature at most 1 has an open regular neighborhood of radius 1 and so the surface has cubical area growth, which we explain as well in this Section. In Section 8 we discuss several Theorems and Conjectures related to flux, including for a properly embedded minimal surface the Flux Conjecture of Meeks and Rosenberg and a related flux conjecture for harmonic functions on parabolic Riemannian manifolds. In Section 9 we cover the aforementioned results of Colding-Minicozzi and the application of their theory to give a sketch of their alternative proof of Collin's Theorem that states that a properly embedded minimal surface of finite topology and at least two ends has finite total curvature. We also explain the important technique of blowingup a sequence of embedded minimal surfaces on the scale of topology, and how this procedure yields a local-global parking garage picture for any embedded genus zero minimal surface in a neighborhood of a point of concentrated topology and more concentrated curvature (this technique was first developed as a tool in [74]). The existence and theory of minimal surfaces with a periodic parking garage structure with a finite number of columns appears in [124]. An important uniqueness for certain parking garage structures was recently given by Weber and Wolf [126], who proved that the columns of these parking garages project to the zeros of Hermite polynomials on the real line; this uniqueness result plays a crucial role in their proposed existence proof of an embedded genus $g$ helicoid for every positive integer $g$. In Section 10 we cover some topological aspects of properly embedded minimal surfaces. These results include a discussion of the recent Topological Classification Theorem for Minimal Surfaces by Frohman and Meeks [38] and the recent topological obstructions of Meeks, Pérez and Ros $[\mathbf{7 5}, \mathbf{7 6}]$ for properly embedded minimal surfaces of finite genus. A particular consequence of these new topological obstructions is that every properly embedded minimal surface of finite genus in $\mathbb{R}^{3}$ is recurrent for Brownian motion. Another important theoretical consequence of these topological obstructions is that a properly embedded minimal surface in $\mathbb{R}^{3}$ of finite topology, at least two ends and with a given bound on its genus, has a related bound on its index of stability. We also sketch the proof of the recent Theorem by Meeks and Rosenberg [80] of the uniqueness of the plane and helicoid as the only properly embedded simply connected minimal surfaces in $\mathbb{R}^{3}$, including their general classification Theorem for minimal laminations of $\mathbb{R}^{3}$ that plays an important theoretical role in many recent advances in the theory. In the final Section 11, we give some partial results on the Genus Zero Conjecture. This Conjecture asserts that a properly embedded minimal surface of genus zero is a plane, a helicoid, a catenoid or an example in the 1-parameter family of minimal surfaces called the Riemann minimal examples (see Subsection 2.5), which are foliated by circles and straight lines in horizontal planes. The solution of this Conjecture reduces to a study of bounded Jacobi functions on properly embedded minimal surfaces $M$ of genus zero with two limit ends. In this Section we define and study the classical Shiffman Jacobi function which is defined on such an $M$ in order to obtain some partial results on the Genus Zero Conjecture.

The authors would like to thank the referee for helpful comments. 


\section{Basic results in classical minimal surface theory.}

We will devote this Section to give a fast tour through the foundations of the theory, enough to understand and apply the results to be explained in future Sections.

2.1. Definitions and Theorems on equivalent properties. One can define a minimal surface from different points of view. The equivalences between these starting points give insight into the richness of the classical theory of minimal surfaces and its connections with other branches of Mathematics.

Definition 1. Let $X: M \rightarrow \mathbb{R}^{3}$ be an isometric immersion of a Riemannian surface into space. $X$ is said to be minimal if the coordinate functions $x_{1}, x_{2}, x_{3}$ are harmonic functions on $M$.

Very often, it is useful to identify a Riemannian surface $M$ with its image under an isometric embedding. Since harmonicity is a local concept, the notion of minimality can be applied to a surface $M \subset \mathbb{R}^{3}$ (with the underlying induced Riemannian structure by the inclusion). Let $H$ be the mean curvature function of $X$ and $N: M \rightarrow \mathbb{S}^{2} \subset \mathbb{R}^{3}$ its Gauss map ${ }^{1}$. The well-known formula $\Delta X=2 H N$, valid for an isometric immersion $X: M \rightarrow \mathbb{R}^{3}$, leads us to the following equivalent definition of minimality.

Definition 2. A surface $M \subset \mathbb{R}^{3}$ is minimal if and only if its mean curvature vanishes identically.

Recall that any (regular) surface can be locally expressed as the graph of a function $u=u(x, y)$. The condition on the mean curvature to vanish identically can be expressed as a quasilinear elliptic second order partial differential equation,

$$
\left(1+u_{x}^{2}\right) u_{y y}-2 u_{x} u_{y} u_{x y}+\left(1+u_{y}^{2}\right) u_{x x}=0 .
$$

Definition 3. A surface $M \subset \mathbb{R}^{3}$ is minimal if and only if it can be locally expressed as the graph of a solution of the equation (1).

Let $\Omega$ be a relatively compact subdomain in a surface $M \subset \mathbb{R}^{3}$. If we perturb normally the inclusion map on $\Omega$ by a compactly supported smooth function $u \in$ $C_{0}^{\infty}(\Omega)$, then $X+t u N$ is again an immersion for any $|t|<\varepsilon$ with $\varepsilon$ sufficiently small. The mean curvature function of $M$ is closely related to the infinitesimal variation of the area for compactly supported normal variations by means of the first variation of area (see for instance $[\mathbf{9 7}]$ ):

$$
A^{\prime}(0)=\left.\frac{d}{d t}\right|_{t=0} \operatorname{Area}((X+t u N)(\Omega))=-2 \int_{\Omega} u H d A,
$$

where $d A$ stands for the area element of $M$. This variational formula lets us state a third equivalent definition of minimality.

Definition 4. A surface $M \subset \mathbb{R}^{3}$ is minimal if and only if it is a critical point of the area functional for all compactly supported variations.

In fact, a consequence of the second variation of area (Subsection 2.8) is that any point in a minimal surface has a neighborhood with least area relative to its boundary. This property justifies the word "minimal" for this kind of surfaces. It

\footnotetext{
${ }^{1}$ Throughout the paper, all surfaces will be assumed to be orientable.
} 
should be noted that the global minimization of area on any compact subdomain is a strong condition for a complete orientable minimal surface to satisfy; in fact, it forces the surface to be a plane (Theorem 8).

Definition 5. A surface $M \subset \mathbb{R}^{3}$ is minimal if and only if every point $p \in M$ has a neighborhood with least area relative to its boundary.

Definitions 4 and 5 establish minimal surfaces as the 2-dimensional analogy to geodesics in Riemannian Geometry, and connect the theory of minimal surfaces with one of the classical important branches of Mathematics: the Calculus of Variations. Besides the area functional $A$, another well-known functional in the Calculus of Variations is the Dirichlet energy,

$$
E=\int_{\Omega}|\nabla X|^{2} d A
$$

where again $X: M \rightarrow \mathbb{R}^{3}$ is an isometric immersion and $\Omega \subset M$ is a subdomain with compact closure. These functionals are related by the inequality $E \geq 2 \mathrm{~A}$, with equality if and only if the immersion $X: M \rightarrow \mathbb{R}^{3}$ is conformal. The classical formula $K-e^{2 u} \bar{K}=\Delta u$ that relates the Gaussian curvature functions $K, \bar{K}$ for two conformally related metrics $g, \bar{g}$ on a 2-dimensional manifold ( $\Delta$ stands for the Laplacian with respect to $g$ ) together with the existence of solutions of the Laplace equation $\Delta u=K$ for a relatively compact subdomain in a Riemannian manifold, guarantee the existence of local isothermal or conformal coordinates for any 2-dimensional Riemannian manifold, modeled on domains of $\mathbb{C}$. The relation between area and energy together with the existence of isothermal coordinates, allow us to give two further characterizations of minimality.

Definition 6. A conformal immersion $X: M \rightarrow \mathbb{R}^{3}$ is minimal if and only if it is a critical point of the Dirichlet energy for compactly supported variations, or equivalently if any point $p \in M$ has a neighborhood with least energy relative to its boundary.

From a physical point of view, the mean curvature function of a homogeneous membrane separating two media is equal, up to a nonzero multiplicative constant, to the difference between the pressures on the two sides of the surface. When this pressure difference is zero, then the membrane has zero mean curvature. Therefore, soap films in space are physical realizations of the ideal concept of a minimal surface.

Definition 7. A surface $M \subset \mathbb{R}^{3}$ is minimal if and only if every point $p \in$ $M$ has a neighborhood $D_{p}$ which is equal to the unique idealized soap film with boundary $\partial D_{p}$.

If $N: M \rightarrow \mathbb{S}^{2}$ is the Gauss map of $M$, then the tangent space $T_{p} M$ of $M$ at $p \in M$ identifies as subspace of $\mathbb{R}^{3}$ under parallel translation with the tangent space $T_{N(p)} \mathbb{S}^{2}$ to the sphere at $N(p)$, from where one can view the differential $A_{p}=-d N_{p}$ as an endomorphism of $T_{p} M$, called the shape operator. $A_{p}$ is a symmetric linear transformation whose orthogonal eigenvectors are the principal directions of $M$ at $p$, and the corresponding eigenvalues are the principal curvatures of $M$ at $p$. Since the mean curvature function $H$ of $M$ equals the arithmetic mean of such principal curvatures, we deduce that minimality reduces to the expression

$$
A_{p}=-d N_{p}=\left(\begin{array}{cc}
a & b \\
b & -a
\end{array}\right)
$$


in an orthonormal tangent basis. After identification of $N$ with its stereographic projection, the Cauchy-Riemann equations give the next last characterization of minimality.

Definition 8. A surface $M \subset \mathbb{R}^{3}$ is minimal if and only if its stereographically projected Gauss map $g: M \rightarrow \mathbb{C} \cup\{\infty\}$ is a meromorphic function.

Definition 1 and the maximum principle for harmonic functions imply that no compact minimal surfaces in $\mathbb{R}^{3}$ exist. Although the study of compact minimal surfaces with boundary has been intensively developed and dates back to famous problems as the well-known Plateau Problem, in this survey we will focus on the study of complete minimal surfaces (possibly with boundary), in the sense that all geodesics can be indefinitely extended up to the boundary of the surface. A stronger global hypothesis, whose relationship with completeness is an active field of research in minimal surface theory, is presented in the following definition.

Definition 9. A map $f: X \rightarrow Y$ between topological spaces is proper if $f^{-1}(C)$ is compact in $X$ for any compact set $C \subset Y$. A minimal surface $M \subset \mathbb{R}^{3}$ is proper when the inclusion map is proper.

The Gaussian curvature function $K$ of a surface $M \subset \mathbb{R}^{3}$ is the product of its principal curvatures. If $M$ is minimal, then its principal curvatures are oppositely signed and thus, $K$ is nonpositive. Another interpretation of $K$ is the determinant of the shape operator $A_{p}$, or equivalently $|K|$ is the absolute value of the Jacobian for the Gauss map $N$. Adding up the curvature at all points of $M$ (note that this integral may be $-\infty$ or a nonpositive number) we will obtain the same quantity as when computing the negative of the spherical area of $M$ through its Gauss map, counting multiplicities. This quantity is called the total curvature of the minimal surface:

$$
C(M)=\int_{M} K d A=-\operatorname{Area}\left(N: M \rightarrow \mathbb{S}^{2}\right) .
$$

2.2. Weierstrass Representation. Recall that the Gauss map of a minimal surface $M$ can be viewed as a meromorphic function on the underlying Riemann surface. Furthermore, the harmonicity of the third coordinate function $x_{3}$ lets us define (at least locally) its harmonic conjugate function $x_{3}^{*}$; hence, the so called height differential ${ }^{2} d h=d x_{3}+i d x_{3}^{*}$ is a holomorphic differential on $M$. The pair $(g, d h)$ is usually referred to as the Weierstrass data of the minimal surface, and the minimal immersion $X: M \rightarrow \mathbb{R}^{3}$ can be expressed up to translations solely in terms of these data as

$$
X(p)=\Re \int_{p_{0}}^{p}\left(\frac{1}{2}\left(\frac{1}{g}-g\right), \frac{i}{2}\left(\frac{1}{g}+g\right), 1\right) d h,
$$

where $\Re$ stands for real part $[\mathbf{5 8 , 9 9}$. The pair $(g, d h)$ satisfies certain compatibility conditions, stated in assertions $i$ ), ii) below. The key point is that the procedure has the following converse, which gives a cook-book type recipe for analytically defining a minimal surface.

Theorem 1 (Osserman [98]). Let $M$ be a Riemann surface, $g: M \rightarrow \mathbb{C} \cup\{\infty\}$ a meromorphic function and dh a holomorphic one-form on $M$. Assume that:

\footnotetext{
${ }^{2}$ Note that the height differential might not be exact since $x_{3}^{*}$ needs not to be globally welldefined on $M$. Nevertheless, the notation $d h$ is commonly accepted and we will also make use of it here.
} 
i) The zeros of dh coincide with the poles and zeros of $g$, with the same order.

ii) For any closed curve $\gamma \subset M$,

$$
\overline{\int_{\gamma} g d h}=\int_{\gamma} \frac{d h}{g}, \quad \Re \int_{\gamma} d h=0 .
$$

Then, the map $X: M \rightarrow \mathbb{R}^{3}$ given by (4) is a conformal minimal immersion with Weierstrass data $(g, d h)$.

Condition i) above expresses the non degeneracy of the induced metric by $X$ on $M$, so by replacing it with the condition that the zeros and poles of $g$ coincide with the zeros of $d h$ with at most the same order, we allow the conformal $X$ to be a branched minimal surface. Condition ii) deals with the independence of (4) on the integration path, and it is usually called the period problem. By Cauchy's Theorem, it suffices to consider the period problem on homology classes in $M$.

All geometric invariants of a minimal surface $M$ can be expressed in terms of its Weierstrass data. For instance, the first and second fundamental forms are respectively (see $[44,99])$ :

$$
d s^{2}=\left(\frac{1}{2}\left(|g|+|g|^{-1}\right)|d h|\right)^{2}, \quad I I(v, v)=\Re\left(\frac{d g}{g}(v) \cdot d h(v)\right),
$$

where $v$ is a tangent vector to $M$, and the Gaussian curvature is

$$
K=-\left(\frac{4|d g / g|}{\left(|g|+|g|^{-1}\right)^{2}|d h|}\right)^{2} .
$$

If $(g, d h)$ is the Weierstrass pair of a minimal surface $X: M \rightarrow \mathbb{R}^{3}$, then for each $\lambda>0$ the pair $(\lambda g, d h)$ satisfies condition $i)$ of Theorem 1 and the second equation in (5). The first equation in (5) holds for these new Weierstrass data if and only if $\int_{\gamma} g d h=\int_{\gamma} \frac{d h}{g}=0$ for all homology classes $\gamma$ in $M$, which in turn is equivalent to the fact that the flux of $M$ along $\gamma$ is vertical for all such $\gamma$. In general, the flux vector is defined as

$$
F(\gamma)=\int_{\gamma}\left(\nabla x_{1}, \nabla x_{2}, \nabla x_{3}\right)=\Im \int_{\gamma}\left(\frac{1}{2}\left(\frac{1}{g}-g\right), \frac{i}{2}\left(\frac{1}{g}+g\right), 1\right) d h,
$$

where $\Im$ stands for imaginary part. Thus, for a minimal surface $X$ with vertical flux, the Weierstrass data $(\lambda g, d h)$ produce a well-defined minimal surface $X_{\lambda}: M \rightarrow \mathbb{R}^{3}$. The family $\left\{X_{\lambda}\right\}_{\lambda}$ is a smooth deformation of $X_{1}=X$, called the López-Ros deformation. Clearly, the conformal structure, height differential and the set of points in $M$ with vertical normal vector are preserved throughout this deformation. Another important property of this deformation is that if a component of a horizontal section of $X$ is convex, then the same holds for the related component at the same height for any $X_{\lambda}, \lambda>0$.

2.3. Minimal surfaces with finite total curvature. Among the family of complete minimal surfaces in space, those with finite total curvature have been extensively studied. The principal reason for this is that they can be thought of as compact algebraic objects in a natural sense, which opens tremendously the number and depth of tools that can be applied to these kinds of surfaces.

Theorem 2 (Huber [52], Osserman [99]). Let $M \subset \mathbb{R}^{3}$ be a complete oriented immersed minimal surface with finite total curvature. Then, 
i) $M$ is conformally a compact Riemann surface $\bar{M}$ with a finite number of points removed (called the ends of $M$ ).

ii) The Weierstrass data $(g, d h)$ extend meromorphically to $\bar{M}$. In particular, the total curvature of $M$ is a multiple of $-4 \pi$.

In this setting, the Gauss map $g$ has a well-defined finite degree on $\bar{M}$. A direct consequence of (3) is that the total curvature of an $M$ as in Theorem 2 is $-4 \pi$ times the degree of its Gauss map $g$. It turns out that this degree can be computed in terms of the genus of the compactification $\bar{M}$ and the number of ends by means of the Jorge-Meeks formula [53]. Rather that stating here this general formula for an immersed surface $M$ as in Theorem 2, we will emphasize the particular case when all the ends of $M$ are embedded:

$$
\operatorname{deg}(g)=\operatorname{genus}(\bar{M})+\#(\text { ends })-1 .
$$

The asymptotic behavior of a complete embedded minimal surface with finite total curvature is well understood. Schoen $[\mathbf{1 1 8}]$ demonstrated that each embedded end of a complete minimal surface with finite total curvature can be parametrized as a graph over the exterior of a disk in the $\left(x_{1}, x_{2}\right)$-plane with height function

$$
x_{3}\left(x_{1}, x_{2}\right)=a \log r+b+\frac{c_{1} x_{1}+c_{2} x_{2}}{r^{2}}+\mathcal{O}\left(r^{-2}\right),
$$

where $r=\sqrt{x_{1}^{2}+x_{2}^{2}}, a, b \in \mathbb{R}$ and $\mathcal{O}\left(r^{-2}\right)$ denotes a function such that $r^{2} \mathcal{O}\left(r^{-2}\right)$ is bounded as $r \rightarrow \infty$. The coefficient $a$ in (9) is called the logarithmic growth of the end. When $a \neq 0$, the end is called a catenoidal end; if $a=0$, we have a planar end. We use this language since a catenoidal end is asymptotic to one of the ends of a catenoid and a planar end is asymptotic to the end of a plane. In particular, complete embedded minimal surfaces with finite total curvature are always proper; in fact, an elementary analysis of the asymptotic behavior shows that the equivalence between completeness and properness still holds for immersed minimal surfaces with finite total curvature.

A key result, proved by Collin in 1997, reduces the study of properly embedded minimal surfaces with finite topology and at least two ends (see Subsection 2.7 for the general definition of end) to the family of surfaces with finite total curvature.

TheOREM 3 (Collin [25]). If $M \subset \mathbb{R}^{3}$ is a properly embedded minimal surface with and more than one end, then each annular end of $M$ is asymptotic to the end of a plane or a catenoid. In particular, if $M$ has finite topology and more than one end, then $M$ has finite total curvature.

The understanding of generic properties of minimal surfaces with finite total curvature leads to existence and uniqueness results, as well as to study the moduli spaces of such surfaces with a fixed topology. Along these lines, Schoen [118] proved in 1983 that the catenoid is the unique complete immersed minimal surface with finite total curvature and two embedded ends. Eight years later, López and Ros [63] characterized the plane and the catenoid as the only complete embedded minimal surfaces in $\mathbb{R}^{3}$ with genus zero and finite total curvature. The most celebrated complete minimal surface with finite total curvature since the classical examples from the nineteenth century was discovered in 1982 by Costa $[\mathbf{2 7 , 2 8}$. This is a thrice punctured torus with two catenoidal ends and one planar middle end. Costa demonstrated existence of this surface but only its embeddedness outside a ball in $\mathbb{R}^{3}$. Hoffman and Meeks [48] proved global embeddedness for the 
Costa torus and generalized this example to any genus $g \geq 2$ and three ends [49]. Also Hoffman and Meeks found a 1-parameter deformation of the Costa surface and of all of the higher genus Hoffman-Meeks minimal surfaces, where the middle planar end becomes catenoidal (unpublished). In [44], Hoffman and Karcher showed that the Hoffman-Meeks deformation families of the Costa-Hoffman-Meeks surfaces exist for all parameter values and that for genus $g>1$, the deformed surfaces are embedded (see Subsection 2.5 for explicit formulas). This embeddedness is equivalent to showing that the logarithmic growth rate of the middle end never catches up with that of the other ends. They also mention that the situation in the case $g=1$ (for the deformation of the Costa torus) is slightly more delicate (and still true), because the aforementioned growth rate of the middle end achieves the growth rate of the extreme ends in the limit. They do not provide this last computation in [44]. Costa $[\mathbf{2 9}, \mathbf{3 0}]$ showed that any complete embedded minimal surface in $\mathbb{R}^{3}$ with genus one and three ends has to be either the Costa surface or lies in the HoffmanMeeks deformation, with the moduli space of complete embedded minimal thrice punctured tori being diffeomorphic to a real interval. Concerning moduli spaces of minimal surfaces with finite total curvature and prescribed topology, Pérez and Ros [106] gave general conditions on the space $\mathcal{M}(g, r)$ whose elements are the complete embedded minimal surfaces with finite total curvature, genus $g$ and $r$ ends, to have a structure of real analytic manifold of dimension $r-2$ around a given minimal surface $M \in \mathcal{M}(g, r)$. Such conditions are expressed in terms of the bounded Jacobi functions on $M$ (see Subsection 2.8 for the definition of Jacobi function). They also identified the tangent space of $\mathcal{M}(g, r)$ at a minimal surface $M \in \mathcal{M}(g, r)$ with the set of Jacobi functions on $M$ with at most logarithmic singularities at the ends. Other compactness results for moduli spaces of complete embedded minimal surfaces with finite total curvature have been given in Ros [112] and Traizet [122]. We will explain in Section 10.2 some further recent advances in this area, see specifically Theorem 29 .

2.4. Periodic minimal surfaces. A properly embedded minimal surface $M$ in $\mathbb{R}^{3}$ is called singly, doubly or triply periodic when it is invariant by a discrete infinite group $G$ of isometries of $\mathbb{R}^{3}$ of rank $1,2,3$ (respectively) that acts properly and discontinuously. Very often, it is useful to study such an $M$ as a minimal surface in the complete flat three manifold $\mathbb{R}^{3} / G$. Up to finite coverings, these 3 -manifolds reduce to $\mathbb{R}^{3} / T, \mathbb{R}^{3} / S_{\theta}, \mathbb{T}^{2} \times \mathbb{R}$ and $\mathbb{T}^{3}$, where $T$ denotes a nontrivial translation, $S_{\theta}$ is the screw motion symmetry resulting from the composition of a rotation of angle $\theta$ around the $x_{3}$-axis with a translation in the direction of this axis, and $\mathbb{T}^{2}, \mathbb{T}^{3}$ are flat tori of dimensions 2 and 3 obtained as quotients of $\mathbb{R}^{2}, \mathbb{R}^{3}$ by 2 or 3 linearly independent translations.

All known periodic minimal surfaces turn out to have finite total curvature (hence finite topology) when seen as surfaces in the corresponding $\mathbb{R}^{3} / G$. Meeks and Rosenberg $[\mathbf{8 1}, \mathbf{8 4}]$ developed the theory of periodic minimal surfaces. For instance, they obtained in this setting similar conclusions as the ones in Theorem 2, except that the Gauss map $g$ of a minimal surface in $\mathbb{R}^{3} / G$ is not necessarily well-defined (the Gauss map does not descend to the quotient for surfaces in $\mathbb{R}^{3} / S_{\theta}, \theta \in(0,2 \pi)$, and in this case the role of the Gauss map $g$ is played by the well-defined differential

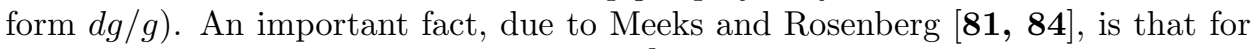
properly embedded minimal surfaces in $\mathbb{R}^{3} / G, G \neq\{$ identity $\}$, the conditions of 
finite total curvature and finite topology are equivalent ${ }^{3}$. Later Meeks $[\mathbf{7 2}]$ proved that every properly embedded minimal surface in $\mathbb{T}^{2} \times \mathbb{R}$ has a finite number of ends, hence in this setting finite genus implies finite total curvature. Analogous to the Jorge-Meeks formula, Meeks and Rosenberg $[\mathbf{8 1}, \mathbf{8 4}]$ proved an explicit relation between the total curvature and the topology of such a surface. They also studied the asymptotic behavior of complete embedded minimal surfaces with finite total curvature in $\mathbb{R}^{3} / G$. In this setting, there are three possibilities: all ends must be simultaneously asymptotic to planes (as in the Riemann minimal examples, see Subsection 2.5), to halfplanes (as in the singly or doubly periodic Scherk minimal surfaces; for this reason, such ends are called Scherk-type ends) or to ends of helicoids (helicoidal type ends). Recently Meeks [66] proved that a properly embedded minimal surface in $\mathbb{R}^{3} / S_{\theta}, \theta \neq 0, \pi$, has a finite number of ends and if it has at least two ends, then the surface has at most quadratic area growth.

Concerning classification theorems for periodic minimal surfaces, Meeks, Pérez and Ros [78] proved that the classical Riemann minimal examples are the unique periodic nonsimply connected genus zero properly embedded minimal surfaces in $\mathbb{R}^{3}$. Lazard-Holly and Meeks [59] characterized the doubly periodic Scherk surfaces as the unique properly embedded examples of genus zero in $\mathbb{T}^{2} \times \mathbb{R}$.

2.5. Examples of minimal surfaces. We will use the Weierstrass representation for introducing some of the most celebrated complete minimal surfaces.

The Plane. $M=\mathbb{C}, g(z)=1, d h=d z$. It is the only flat minimal surface.

The Catenoid. $M=\mathbb{C}-\{0\}, g(z)=z, d h=\frac{d z}{z}$. It has genus zero, two ends and total curvature $-4 \pi$. Together with the plane, the catenoid is the only minimal surface of revolution (Bonnet [4]) and the unique complete embedded minimal surface with genus zero and finite total curvature (López and Ros [63]). Schoen [118] also characterized the catenoid as the unique complete immersed minimal surface with finite total curvature and two embedded ends.

The Helicoid. $M=\mathbb{C}, g(z)=e^{z}, d h=i d z$. It has genus zero, one end and infinite total curvature. Together with the plane, the helicoid is the only ruled minimal surface (Catalan [8]) and the unique properly embedded simply connected minimal surface (Meeks and Rosenberg [80], see also Theorem 30 below). The vertical helicoid can be also seen as a genus zero surface with two ends in a quotient of $\mathbb{R}^{3}$ by a vertical translation or by a screw motion. The catenoid and the helicoid are conjugate minimal surfaces, in the sense that the coordinate functions of one of these surfaces are the harmonic conjugates of the coordinate functions of the other one; in this case, we consider the catenoid to be defined on its universal cover $e^{z}: \mathbb{C} \rightarrow \mathbb{C}-\{0\}$ in order for the harmonic conjugate of $x_{3}$ to be well-defined. Equivalently, both surfaces share the Gauss map $e^{z}$ and their height differentials differ in multiplication by $i=\sqrt{-1}$.

The Enneper surface. $M=\mathbb{C}, g(z)=z, d h=z d z$. It is nonembedded, has genus zero, one end and total curvature $-4 \pi$. The catenoid and the Enneper surface are the unique complete minimal surfaces in $\mathbb{R}^{3}$ with finite total curvature $-4 \pi$ (see $[\mathbf{9 9}]$ ).

Given $k \in \mathbb{N}, k \geq 1$ and $a \in \mathbb{R}-\{0,-1\}$, we define the compact genus $k$ surface $\bar{M}_{k, a}=\left\{(z, w) \in(\mathbb{C} \cup\{\infty\})^{2} \mid w^{k+1}=\frac{(z+1)(z-a)}{z}\right\}$. Let $M_{k, a}=\bar{M}_{k, a}-$

\footnotetext{
${ }^{3}$ This equivalence does not hold for properly embedded minimal surfaces in $\mathbb{R}^{3}$, as demon-
} strates the helicoid. 
$\{(-1,0),(\infty, \infty),(a, 0)\}$ and

$$
g_{k, a, m, A}(z, w)=A \frac{z w}{m z+1}, \quad d h_{k, a, m}=\frac{m z+1}{(z+1)(z-a)} d z,
$$

where $A \in \mathbb{R}-\{0\}$. Given $k \in \mathbb{N}$ and $a \in(0, \infty)$, there exist $m=m(a) \in \mathbb{R}$ and $A=A(a) \in \mathbb{R}-\{0\}$ such that the pair $\left(g_{k, a, m(a)}, A(a), d h_{k, a, m(a)}\right)$ is the Weierstrass data of a well-defined minimal surface $X: M_{k, a} \rightarrow \mathbb{R}^{3}$ with genus $k$ and three ends (Hoffman, Karcher [44]). Moreover, $m(1)=0$ for any $k \in \mathbb{N}$. With this notation, we have the following examples.

The Costa torus. $M=M_{1,1}, g=g_{1,1,0, A(1)}, d h=d h_{1,1,0}$. Costa [28] proved existence of this surface, while its embeddedness is due to Hoffman and Meeks [48]. The Costa-Hoffman-Meeks surfaces. For any $k \geq 2$, take $M=M_{k, 1}, g=$ $g_{1,1,0, A(1)}, d h=d h_{1,1,0}$. Both existence and embeddedness were given by Hoffman and Meeks $[49]$.

The Deformation of the Costa torus. For any $a \in(0, \infty)$, take $M=M_{1, a}$, $g=g_{1, a, m(a), A(a)}, d h=d h_{1, a, m(a)}$ (when $a=1$ we find the Costa torus). Hoffman and Karcher [44] proved existence of these surfaces. A complete proof of their embeddedness has not been published yet, see also the last paragraph of Subsection 2.3 and $[\mathbf{4 5}]$. Costa $[\mathbf{2 9}, \mathbf{3 0}]$ showed that any complete embedded minimal torus with three ends must lie in this family.

The Deformation of the Costa-Hoffman-Meeks surfaces. For any $k \geq 2$ and $a \in(0, \infty)$, take $M=M_{k, a}, g=g_{k, a, m(a), A(a)}, d h=d h_{k, a, m(a)}$. When $a=1$ we find the Costa-Hoffman-Meeks surface of genus $k$ and three ends. A complete proof of existence and embeddedness for these surfaces is given in [44] by Hoffman and Karcher.

The Singly PERIODIC SCherk Surfaces. $M=(\mathbb{C} \cup\{\infty\})-\left\{ \pm e^{ \pm i \theta}\right\}, g(z)=z$, $d h=\frac{i z d z}{\prod\left(z \pm e^{ \pm i \theta}\right)}$, for fixed $\theta \in(0, \pi / 4]$. Discovered (at least, the case $\left.\theta=\pi / 4\right)$ by Scherk [116] in 1835, they form a 1-parameter family of genus zero surfaces in a quotient of $\mathbb{R}^{3}$ by a translation, with four ends. Each surface can be thought of geometrically as a desingularization of two vertical planes forming an angle of $2 \theta$.

The doubly Periodic Scherk surfaces. $M=(\mathbb{C} \cup\{\infty\})-\left\{ \pm e^{ \pm i \theta}\right\}, g(z)=z$, $d h=\frac{z d z}{\prod\left(z \pm e^{ \pm i \theta}\right)}$, where $\theta \in(0, \pi / 4]$. These are the conjugate surfaces to the singly periodic Scherk surfaces, and can be thought of geometrically as the desingularization of two families of equally spaced vertical parallel halfplanes in opposite halfspaces, with the halfplanes in the upper family making an angle of $2 \theta$ with the halfplanes in the lower family. These surfaces are doubly periodic with genus zero in their corresponding quotient $\mathbb{T}^{2} \times \mathbb{R}$, and were characterized by Lazard-Holly and Meeks [59] as the unique properly embedded minimal surfaces in $\mathbb{T}^{2} \times \mathbb{R}$ with genus zero.

The Riemann minimal examples. $M=\left\{(z, w) \in(\mathbb{C} \cup\{\infty\})^{2} \mid w^{2}=z(z-\right.$ $\lambda)(\lambda z+1)\}-\{(0,0),(\infty, \infty)\}, g(z, w)=z, d h=A_{\lambda} \frac{d z}{w}$, for each $\lambda>0$, where $A_{\lambda}$ is a nonzero complex number satisfying $A_{\lambda}^{2} \in \mathbb{R}$. Discovered by Riemann (and posthumously published, Hattendorf and Riemann $[\mathbf{1 1 0}, \mathbf{1 1 1}]$ ), these surfaces are invariant by a translation $T_{\lambda}$, and in the quotient space $\mathbb{R}^{3} / T_{\lambda}$ have genus one and two planar ends. The conjugate surface of the Riemann minimal example for a given $\lambda>0$ is the Riemann minimal example for the parameter value $1 / \lambda$ (the case $\lambda=1$ gives the only self-conjugate surface in the family). Riemann minimal examples were characterized by Meeks, Pérez and Ros [78] as the unique periodic nonsimply connected genus zero properly embedded minimal surfaces in $\mathbb{R}^{3}$. 
2.6. Monotonicity formula and classical maximum principles. As we will see in Section 6, the conformal type of a minimal surface is strongly related with its area growth in balls. The first result along these lines comes from the coarea formula applied to the distance function to a given point $p \in \mathbb{R}^{3}$. The following statement of the coarea formula appears in $[\mathbf{9}]$, see $[\mathbf{3 3}]$ for a more general version.

Proposition 1. Let $\Omega$ be a domain with compact closure in a Riemannian manifold $M$ and $f: \bar{\Omega} \rightarrow \mathbb{R}$ a function in $C^{0}(\bar{\Omega}) \cap C^{\infty}(\Omega)$ with $\left.f\right|_{\partial \Omega}=0$. For any regular value $t$ of $|f|$, we let $\Gamma(t)=|f|^{-1}(t)$ and $A(t)=\operatorname{Area}(\Gamma(t))$. Then for any function $\phi \in L^{1}(\Omega)$ we have

$$
\int_{\Omega} \phi|\nabla f| d V=\int_{0}^{\infty}\left(\int_{\Gamma(t)} \phi d A_{t}\right) d t,
$$

where $\nabla f$ is the gradient of $f$ in $M$ and $d V, d A_{t}$ are respectively the volume elements in $M$ and $\Gamma(t)$.

TheOREM 4 (Monotonicity formula $[\mathbf{2 0}, \mathbf{5 5}]$ ). Let $X: M \rightarrow \mathbb{R}^{3}$ be a properly immersed connected minimal surface. Given $p \in \mathbb{R}^{3}$, let $A(R)$ be the area of the portion of $X(M)$ inside a ball of radius $R>0$ centered at $p$. Then, $A(R) R^{-2}$ is nondecreasing. In particular, $\lim _{R \rightarrow \infty} A(R) R^{-2} \geq \pi$ with equality if and only if $M$ is a plane.

One of the consequences of the fact that minimal surfaces can be viewed locally as solutions of the partial differential equation (1) is the validity of certain maximum principles for minimal surfaces. We will state them for minimal surfaces in $\mathbb{R}^{3}$, but they also hold when the ambient space is a complete flat three-manifold.

TheOREM 5 (Interior maximum principle [118]). Let $M_{1}, M_{2}$ be connected minimal surfaces in $\mathbb{R}^{3}$ and $p$ a point interior to both surfaces, such that $T_{p} M_{1}=$ $T_{p} M_{2}=\left\{x_{3}=0\right\}$. If $M_{1}, M_{2}$ are locally expressed as the graphs of functions $u_{1}, u_{2}$ around $p$ and $u_{1} \leq u_{2}$ in a neighborhood of $p$, then $M_{1}=M_{2}$ in a neighborhood of $p$.

Theorem 6 (Maximum principle at infinity $[\mathbf{8 2}, \mathbf{5 6}]$ ). Let $M_{1}, M_{2} \subset N^{3}$ be disjoint connected properly immersed minimal surfaces with compact (possibly empty) boundary in a complete flat 3-manifold $N^{3}$.

i) If $\partial M_{1} \neq \varnothing$ or $\partial M_{2} \neq \varnothing$, then after possibly reindexing, there exist points $p \in \partial M_{1}, q \in M_{2}$ such that $\operatorname{dist}(p, q)=\operatorname{dist}\left(M_{1}, M_{2}\right)$.

ii) If $\partial M_{1}=\partial M_{2}=\varnothing$, then $M_{1}$ and $M_{2}$ are flat.

The maximum principle at infinity can be generalized to the case of noncompact boundaries, see Theorem 21 below. A beautiful application of Theorem 5 is the following result by Hoffman and Meeks.

TheOREm 7 (Halfspace Theorem [50]). A proper, connected, possibly branched, nonplanar minimal surface $M \subset \mathbb{R}^{3}$ cannot be contained in a halfspace.

For later reference in the survey, we next provide a sketch of the original direct proof of Theorem 7. Arguing by contradiction, suppose that $M \subset H=\left\{x_{3} \geq 0\right\} \subset$ $\mathbb{R}^{3}$ is a connected, properly immersed, nonflat minimal surface. By the interior maximum principle, $M \subset \operatorname{Int}(H)$. After a suitable vertical translation, we can assume that $\operatorname{dist}(M, \partial H)=0$. Let $C=\left\{\left(x_{1}, x_{2}, x_{3}\right) \mid x_{1}^{2}+x_{2}^{2}=\cosh ^{2} x_{3}, x_{3}<0\right\}$ be the lower half of a vertical catenoid. Since $M$ is proper and disjoint from the 
$\left(x_{1}, x_{2}\right)$-plane, there exists $\varepsilon>0$ such that $\left(M-\varepsilon e_{3}\right) \cap C=\varnothing$ and $\left(M-\varepsilon e_{3}\right) \cap(\mathbb{D} \times$ $[-1,0])=\varnothing$, where $e_{3}=(0,0,1)$ and $\mathbb{D}$ is the unit disk in $\partial H$. Now consider the homothetically shrunk halfcatenoids $C_{t}=t C, 0<t \leq 1$. As $t \rightarrow 0, C_{t}$ converges smoothly to $\partial H-\{0\}$ away from the origin. It follows that for $t>0$ sufficiently small, $\left(M-\varepsilon e_{3}\right) \cap C_{t} \neq \varnothing$. From here it is not difficult to prove that there exists a largest $t_{0} \in(0,1]$ such that $\left(M-\varepsilon e_{3}\right) \cap C_{t_{0}} \neq \varnothing$. Since the intersection $\left(M-\varepsilon e_{3}\right) \cap C_{t_{0}}$ occurs outside the cylinder $\mathbb{D} \times[-1,0]$, we find $p \in\left(M-\varepsilon e_{3}\right) \cap C_{t_{0}}$ which is interior to both surfaces. Since the translated surface $M-\varepsilon e_{3}$ is above $C_{t_{0}}$ around $p$, the interior maximum principle insures that $M-\varepsilon e_{3}=C_{t_{0}}$, which is a contradiction.

2.7. Ends of properly embedded minimal surfaces. One of the fundamental problems in classical minimal surface theory is to describe the behavior of a properly embedded minimal surface $M \subset \mathbb{R}^{3}$ outside a large compact set in space. This problem is well understood if $M$ has finite total curvature (see Theorem 2), because each of its ends is asymptotic to an end of a plane or a catenoid. A recent Theorem by Meeks and Rosenberg [80] proves that if $M$ has finite topology but infinite total curvature, then $M$ is asymptotic to a helicoid (Theorem 30). More complicated asymptotic behaviors can be found in periodic minimal surfaces in $\mathbb{R}^{3}$, although this asymptotic behavior is completely understood when the periodic minimal surface has finite topology (hence finite total curvature) in the corresponding quotient ambient space; in this setting, only planar, helicoidal or Scherk-type ends can occur (Meeks and Rosenberg $[\mathbf{8 1}, \mathbf{8 4}]$ ).

A crucial notion in the understanding of the asymptotic geometry of a generic properly embedded minimal surface is the notion of topological end, which we now explain. Let $M$ be a noncompact connected manifold. We define an equivalence relation in the set $\mathcal{A}=\{\alpha:[0, \infty) \rightarrow M \mid \alpha$ is a proper arc $\}$ by setting $\alpha_{1} \sim \alpha_{2}$ if for every compact set $C \subset M, \alpha_{1}, \alpha_{2}$ lie eventually ${ }^{4}$ in the same component of $M-C$.

Definition 10. Each equivalence class in $\mathcal{E}(M)=\mathcal{A} / \sim$ is called an end of $M$. If $e \in \mathcal{E}(M), \alpha \in e$ is a representative proper arc and $\Omega \subset M$ is a proper subdomain containing $\alpha$ with compact boundary, then we say that the domain $\Omega$ represents the end $e$.

$\mathcal{E}(M)$ has the following natural Hausdorff topology. For each proper domain $\Omega \subset M$ with compact boundary, we define the basis open set $B(\Omega) \subset \mathcal{E}(M)$ to be those equivalence classes in $\mathcal{E}(M)$ which have representatives contained in $\Omega$. With this topology, $\mathcal{E}(M)$ is a totally disconnected compact space which embeds topologically as a subspace of $[0,1] \subset \mathbb{R}$. Since this result is not known, or at least its proof does not seem to appear in the literature, we give a short proof of it at the end of this Subsection.

Definition 11. Any isolated point $e \in \mathcal{E}(M)$ is called a simple end of $M$. If $e \in \mathcal{E}(M)$ is not a simple end (equivalently, if it is a limit point of $\mathcal{E}(M) \subset[0,1]$ ), we will call it a limit end of $M$.

When $M$ has dimension 2, then an end $e \in \mathcal{E}(M)$ is simple if and only if it can be represented by a proper subdomain $\Omega \subset M$ with compact boundary which is

\footnotetext{
${ }^{4}$ Throughout the paper, eventually for proper arcs means outside a compact subset of the parameter domain $[0, \infty)$.
} 
homeomorphic to $\mathbb{S}^{1} \times[0, \infty)\left(\right.$ this case is called an annular end) or to $\mathbb{S}^{1} \times[0, \infty)$ connected sum with an infinite number of tori where the $n$-th connected sum occurs at the point $(1, n) \in \mathbb{S}^{1} \times[0, \infty), n \in \mathbb{N}$ (this is a simple end of infinite genus). For limit ends there are similar notions: a limit end $e \in \mathcal{E}(M)$ is said to have genus zero if it can be represented by a proper subdomain $\Omega \subset M$ with compact boundary and genus zero. If a limit end $e$ does not have genus zero, then we say that it has infinite genus; in this case every proper subdomain with compact boundary representing $e$ has infinite genus.

We will devote Section 5 to the Ordering Theorem for ends of properly embedded minimal surfaces; this Theorem is the starting point for the theory of properly embedded minimal surfaces with more than one end. Concerning one-ended minimal surfaces, the classical example in this family is the helicoid. In 1993, Hoffman, Karcher and Wei $[\mathbf{4 6}, 47]$ found a surprising example with genus one and one helicoidal end. Recently, Hoffman, Weber and Wolf [51] have given a proof of the embeddedness of a genus one helicoid, and there are computational indications that point to the existence of a unique embedded example with one helicoidal end for any positive genus (the first computer graphics images of a higher genus helicoid are due to Traizet — unpublished - , see also Bobenko [2], Bobenko and Schmies [3]). From the theoretical point of view, a recent result by Meeks and Rosenberg [80] insures that any properly embedded one-ended minimal surface with finite topology must be necessarily asymptotic to a helicoid with finitely many handles and it can be described analytically by meromorphic data $(d g / g, d h)$ on a compact Riemann surface by means of the classical Weierstrass representation, see Theorem 30 . Regarding one-ended surfaces with infinite topology, Callahan, Hoffman and Meeks [6] showed that any nonflat doubly or triply periodic minimal surface in $\mathbb{R}^{3}$ must have infinite genus and only one end.

We finish this Subsection by proving that the space $\mathcal{E}(M)$ of ends of a noncompact connected manifold $M$ is a totally disconnected compact space that embeds in the unit interval $[0,1]$. Our proof generalizes to many other spaces including finite dimensional simplicial complexes. If $\mathcal{E}(M)$ is a finite set, then the embedding property is obvious. Assume now that $M$ has an infinite number of ends. Let $\Omega_{1} \subset \ldots \subset \Omega_{n} \subset \ldots$ be a compact exhaustion of $M$. It is not difficult to inductively modify this exhaustion to produce a new exhaustion (denoted in the same way) which satisfies the following properties:

1. $\Omega_{n}$ is a manifold with boundary.

2. $\Omega_{n} \subset \operatorname{Int}\left(\Omega_{n+1}\right)$.

3. For $n \geq 2$, every boundary component of $\Omega_{n}$ separates $M$ into two closed noncompact regions.

4. The number of boundary components of $\Omega_{n}$ is $n$.

5. Each end $e \in \mathcal{E}(M)$ has a representative $\alpha$ which begins in $\Omega_{1}$ and intersects transversely each boundary component of $\Omega_{n}$ at most once point.

Consider the closure in $M$ of the two components of $\Omega_{3}-\Omega_{2}$. One of these components, which we label $M_{2}$, has exactly 2 boundary components, while the other component $M_{3}$ has 3 boundary components. This process can be inductively continued to label the closure in $M$ of the $n$ closed components of $\Omega_{n+1}-\Omega_{n}$ as follows. Assume that $\left\{M_{a_{1}, \ldots, a_{n-2}}\right\}$ is the collection of the components of the previous stage. We label a closed component of $\Omega_{n+1}-\Omega_{n}$ by $M_{a_{1}, \ldots, a_{n-1}}$ with 
$a_{n-1} \in\{3,4,5,6,7\}$ so that $M_{a_{1}, \ldots, a_{n-1}}$ attaches to $M_{a_{1}, \ldots, a_{n-2}}$ and one of the following possibilities holds:

1. $a_{n-1}=3$ if $M_{a_{1}, \ldots, a_{n-1}}$ has three boundary components.

2. $a_{n-1}=4$ if $M_{a_{1}, \ldots, a_{n-1}}$ has two boundary components and $M_{a_{1}, \ldots, a_{n-2}}$ has also two boundary components.

3. $a_{n-1}=5$ if $M_{a_{1}, \ldots, a_{n-1}}$ has two boundary components, $M_{a_{1}, \ldots, a_{n-2}}$ has three boundary components and the other component attaching to $M_{a_{1}, \ldots, a_{n-2}}$ has three boundary components.

4. $a_{n-1}=6,7$ if $M_{a_{1}, \ldots, a_{n-1}}$ has two boundary components and it is not in the previous cases.

We now explain how to embed the totally disconnected compact Hausdorff space $\mathcal{E}(M)$ into $[0,1]$ where we consider every point in $[0,1]$ to be expressed as decimal point followed by an infinite sequence of digits. Let $e \in \mathcal{E}(M)$ and take a representative $\alpha \in e$ satisfying property 5 above. Then $\alpha$ eventually lies in $M_{a_{1}} \cup M_{a_{1}, a_{2}} \cup \ldots \cup M_{a_{1}, \ldots, a_{n}} \cup \ldots$ for a unique infinite sequence $S(\alpha)=$ $\left(a_{1}, a_{2}, \ldots, a_{n}, \ldots\right) \in[0,1]$. Note that $S(\alpha)$ is independent of the choice of the proper arc $\alpha \in e$ satisfying property 5 , so we can denote this decimal number as $S(e)$. It is straightforward to prove that the map $e \mapsto S(e)$ is a topological embedding. In the case that $M$ is a properly embedded minimal surface in $\mathbb{R}^{3}$ with more than one end, there is a more natural topological embedding of $\mathcal{E}(M)$ into $[0,1]$ that uses the relative heights of the ends of $M$, see the Ordering Theorem 18 in Section 5.

2.8. Second variation of area and Jacobi functions. Let $M \subset \mathbb{R}^{3}$ be a minimal surface and $\Omega \subset M$ a subdomain with compact closure. Any compactly supported, smooth, normal deformation of the inclusion $X: M \rightarrow \mathbb{R}^{3}$ on $\Omega$ can be written as $X+t u N$, where $N$ is the Gauss map of $M$ and $u \in C_{0}^{\infty}(M)$. By (2), the area functional $A=A(t)$ for this deformation has $A^{\prime}(0)=0$. The second variation of area can be easily shown to be (see $[\mathbf{9 7}]$ )

$$
A^{\prime \prime}(0)=-\int_{\Omega} u(\Delta u-2 K u) d A
$$

where $K$ is the Gaussian curvature function of $M$ and $\Delta$ its Laplace operator. Formula (10) can be seen as the classical bilinear form associated to the linear elliptic $L^{2}$-selfadjoint operator $L=\Delta-2 K=\Delta+|\nabla N|^{2}$, which is usually called the Jacobi operator.

Definition 12. A $C^{2}$-function $u: M \rightarrow \mathbb{R}$ satisfying $\Delta u-2 K u=0$ on $M$ is called a Jacobi function. We will let $\mathcal{J}(M)$ denote the space of Jacobi functions on the minimal surface $M$.

Classical elliptic theory implies that for a given subdomain $\Omega \subset M$ with compact closure, the Dirichlet problem for the Jacobi operator in $\Omega$ has an infinite discrete sequence $\left\{\lambda_{k}\right\}_{k \in \mathbb{N} \cup\{0\}}$ of eigenvalues with $\lambda_{k} \nearrow+\infty$ as $k$ goes to infinity, and each eigenspace is a finite dimensional linear subspace of $C^{\infty}(\Omega) \cap H_{0}^{1}(\Omega)$, where $H_{0}^{1}(\Omega)$ denotes the usual Sobolev space of $L^{2}$ functions with $L^{2}$ weak partial derivatives and trace zero. Since any normal variation through minimal surfaces has vanishing second derivative of the area functional, it follows that the normal parts of variational fields coming from Killing or dilatation vector fields of $\mathbb{R}^{3}$ produce elements in $\mathcal{J}(M)$. For instance, translations give rise to the so called linear Jacobi 
functions $\langle N, v\rangle$ with $v \in \mathbb{R}^{3}$, rotations produce the Jacobi functions $\operatorname{det}(p, N, v)$ (where $p$ denotes the position vector) and homotheties give the support function $\langle p, N\rangle \in \mathcal{J}(M)$. A particularly interesting Jacobi function, which is defined when the minimal surface is transverse to a family of horizontal planes, is the Shiffman function, which will be studied in Section 11.

Definition 13. Let $\Omega \subset M$ be a subdomain with compact closure. The index of stability of $\Omega$ is the number of bounded states of $L$ in such domain, i.e. the number of negative eigenvalues of the Dirichlet problem associated to $L$ in $\Omega$. The nullity of $\Omega$ is the dimension of $\mathcal{J}(\Omega) \cap H_{0}^{1}(\Omega)$. $\Omega$ is called stable if its index is zero, and strictly stable if both its index and nullity are zero.

Elliptic theory also implies that $\Omega$ is strictly stable provided that it is sufficiently small, which justifies the Definition 5 of minimal surface as a local minimum of area. Another consequence of elliptic theory is that $\Omega$ is stable if and only if it carries a positive Jacobi function. Since the Gauss map $N$ of a graph defined on a domain in a plane $\Pi$ has image set contained in an open halfsphere, the inner product of $N$ with the unit normal to $\Pi$ provides a positive Jacobi function, from where we conclude that any minimal graph is stable. Stability makes sense in the large, as we next explain.

Definition 14. A minimal surface $M \subset \mathbb{R}^{3}$ is called stable if any relatively compact subdomain $\Omega \subset M$ is stable. For orientable minimal surfaces, stability is equivalent to the existence of a positive Jacobi function (Proposition 1 in [34]). $M$ is said to have finite index if outside of a compact subset it is stable. The index of stability of $M$ is the supremum of the indices of relatively compact subdomains in $M$.

By definition, stable surfaces have index zero. The following Theorem explains how restrictive is the property of stability for complete minimal surfaces. It was proved independently by Fischer-Colbrie and Schoen [35], do Carmo and Peng [31], and Pogorelov [109].

THEOREM 8. If $M \subset \mathbb{R}^{3}$ is a complete (orientable) immersed stable minimal surface, then $M$ is a plane.

We will provide a short elementary proof of Theorem 8 in Section 4.1. If we weaken the stability hypothesis to finite index, then completeness also leads to a well-known family of minimal surfaces.

THEOREM 9 (Fischer-Colbrie [34]). If $M \subset \mathbb{R}^{3}$ is a complete (orientable) minimal surface with possibly empty compact boundary, then $M$ has finite index if and only if it has finite total curvature. In this case, the index and nullity of $M$ coincides with the index and nullity of the meromorphic extension of its Gauss map to the compactification $\bar{M}$ obtained from $M$ after attaching its ends, see Theorem 2.

By the conformal invariance of the Dirichlet integral, both the index and nullity of the Jacobi operator $L=\Delta+|\nabla N|^{2}$ remain constant under a conformal change of metric. On the other hand, Osserman's Theorem implies that every complete immersed minimal surface $M \subset \mathbb{R}^{3}$ with finite total curvature is conformally equivalent to a finitely punctured compact Riemann surface $\bar{M}$. It can be shown (Pérez and Ros [106]) that there exists a smooth metric $d \bar{s}^{2}$ on the compactification $\bar{M}$ such that the metric $d s^{2}$ on $M$ induced by the inner product of $\mathbb{R}^{3}$ can be expressed 
as $d s^{2}=\mu d \bar{s}^{2}$, where $\mu$ is a positive smooth function that blows up at the ends of $M$. In this setting, both the index and nullity of $L$ can be computed as the index and nullity of the operator $\bar{L}=\bar{\Delta}+|\bar{\nabla} N|^{2}$ on $\bar{M}$ minus the ends, where a bar means that the corresponding object is computed with respect to $d \bar{s}^{2}$. Also by Osserman's Theorem, $N$ extends as a meromorphic function to $\bar{M}$, thus $\bar{L}$ is nothing more than the classical Schrödinger operator associated to such a meromorphic extension. The subspace $\mathcal{K}(M)$ of bounded Jacobi functions on $M$ can be identified with the eigenspace associated to the eigenvalue 0 of the operator $\bar{L}$. Inside $\mathcal{K}(M)$ we have the subspace of linear functions $\mathcal{L}(M)=\left\{\langle N, v\rangle \mid v \in \mathbb{R}^{3}\right\}$. If additionally all the ends of $M$ are horizontal, then $\operatorname{det}\left(p, N, e_{3}\right) \in \mathcal{K}(M)$, where $e_{3}=(0,0,1)$. In particular, $\mathcal{K}(M)$ has dimension at least 4 for any complete embedded minimal surface of finite total curvature in $\mathbb{R}^{3}$ except for the catenoid where $\operatorname{det}\left(p, N, e_{3}\right)$ vanishes.

Montiel and Ros $[\mathbf{9 1}]$ stated a beautiful relationship between bounded Jacobi functions and branched minimal immersions. For a complete minimal surface $M \subset$ $\mathbb{R}^{3}$ with finite total curvature, let $B(N) \subset \bar{M}$ be the set of branch points of the extended Gauss map and $\mathcal{M}(N)$ the linear space of all complete branched minimal immersions (including the constant maps) of $M-B(N)$ into $\mathbb{R}^{3}$ with the same Gauss map $N$ as $M$.

Theorem 10 (Montiel, Ros $[\mathbf{9 1}]$ ). Let $M \subset \mathbb{R}^{3}$ be a complete immersed minimal surface with finite total curvature ${ }^{5}$. Then, there exists a linear map $u \in$ $\mathcal{K}(M) \mapsto X_{u} \in \mathcal{M}(N)$ such that the support function of $X_{u}$ is $u$, and $u \in \mathcal{L}(M)$ if and only if $X_{u}$ is constant. Furthermore, this linear map gives rise to an isomorphism between the quotient spaces $\mathcal{K}(M) / \mathcal{L}(M)$ and $\mathcal{M}(N) /\{$ constants $\}$.

Among the admissible conformal metrics which can be used to express questions related with the Jacobi operator, a particularly interesting choice comes from consideration of the pullback metric $d s_{N}^{2}$ through the Gauss map from the standard spherical metric on $\mathbb{S}^{2}$. The metric $d s_{N}^{2}$ has singularities at the branch points of $N$ and the Jacobi operator transforms into $L_{N}=\Delta_{N}+2$, where $\Delta_{N}$ is the Laplacian of $d s_{N}^{2}$. Eigenvalues and eigenfunctions of $L_{N}$ are well-defined by a variational approach (Tysk [125]). In particular, the index of stability of a relatively compact subdomain $\Omega \subset M$ is equal to the number of eigenvalues of $\Delta_{N}$ which are strictly less than 2 , and the nullity of $\Omega$ is the multiplicity of 2 as an eigenvalue of $\Delta_{N}$. Using these ideas, Montiel and Ros [91] gave some estimates for the index and nullity under different geometrical assumptions, of which we emphasize the following one.

TheOREM 11. Let $M \subset \mathbb{R}^{3}$ be a complete immersed minimal surface with finite total curvature ${ }^{5}$. If all the branch values of the Gauss map of $M$ lie on a equator of $\mathbb{S}^{2}$, then the dimension of $\mathcal{K}(M)$ is 3 .

\section{Conformal questions on minimal surfaces.}

3.1. Recurrence and parabolicity for manifolds. The conformal structure of a complete minimal surface has a strong influence on its global properties. In particular, an important question is to decide the so called type problem for a minimal surface $M$, in the sense of classical Riemann surfaces: i.e. whether $M$

\footnotetext{
${ }^{5}$ Theorems 10 and 11 remain valid for complete minimal surfaces in any quotient of $\mathbb{R}^{3}$ where the Gauss map makes sense, and which have finite total curvature in the quotient.
} 
is hyperbolic or parabolic ${ }^{6}$ (as we have already noticed, the elliptic or compact case is impossible for a minimal surface). It turns out that the parabolicity for Riemann surfaces without boundary is equivalent to the recurrence of Brownian motion of such surfaces. This field lies in the borderline between several branches of Mathematics such as Riemannian Geometry, Stochastic Analysis, Partial Differential Equations and Potential Theory. A particularly interesting source where the reader can find an excellent introduction to these questions is the survey of recurrence and Brownian motion on Riemannian manifolds by Grigor'yan [41]. The goal of this Subsection is to introduce some key concepts that are useful in dealing with these conformal questions. In order to avoid concepts closely related with probability (such as random walks or Brownian motion), in this paper we will follow an alternative way to define recurrence and parabolicity that is slightly different from Grigor'yan's approach; this approach is well-known and is explained in greater detail in the notes by the second author [101]. However, we will briefly explain the connection between these two approaches. We will not provide proofs for most of the results stated in this Subsection, but the proofs can be found in $[\mathbf{4 1}]$ or $[\mathbf{1 0 1}]$.

Definition 15. Let $\left(M^{n}, g\right)$ be a $n$-dimensional Riemannian manifold with nonempty boundary. $M$ is parabolic if every bounded harmonic function on $M$ is determined by its boundary values.

Definition 16. Let $\left(M^{n}, g\right)$ be a $n$-dimensional Riemannian manifold without boundary. $M$ is recurrent if for any nonempty open set $O \varsubsetneqq M$ with smooth boundary, $M-O$ is parabolic.

Given a Riemannian manifold $(M, g)$ with boundary $\partial M \neq \varnothing$ and a point $p \in \operatorname{Int}(M)$, the harmonic measure $\mu_{p}$ with respect to $p$ can be defined as follows. Let $I \subset M$ be a nonempty open set with smooth boundary. Consider a compact exhaustion $I \subset \partial M_{1} \subset M_{1} \subset M_{2} \subset \ldots$ of $M$. Given $k \in \mathbb{N}$, let $h_{k}: M \rightarrow[0,1]$ be the (bounded) harmonic function on $M_{k}$ with boundary values 1 on $\operatorname{Int}(I)$ and 0 on $\partial M_{k}-I$. After extending $h_{k}$ by zero to $M$, we can see $\left\{h_{k}\right\}_{k}$ as an increasing sequence of harmonic functions, bounded from above by 1 . Hence $h_{k}$ limits to a unique bounded harmonic function $h: M \rightarrow[0,1]$. In this situation, we define $\mu_{p}(I)=h(p)$. It turns out that $\mu_{p}$ extends to a Borel measure $\mu_{p}$ on $\partial M$.

Another interpretation of $\mu_{p}$, developed in $[\mathbf{4 1}]$, is that $\mu_{p}(I)$ is the probability of a Brownian path beginning at $p$, of hitting $\partial M$ the first time somewhere on the interval $I$, and for this reason the harmonic measure of $M$ is also called the hitting measure with respect to $p$. We now explain how to computationally calculate the hitting measure $\mu_{p}$ at an interval $I$ contained in the boundary of a smooth domain $\Omega \subset \mathbb{R}^{2}$, where $p \in \operatorname{Int}(\Omega)$. For $n \in \mathbb{N}$ and $\varepsilon>0$, define the set $\Gamma(p, n, \varepsilon)$ to be the $n$-step orthogonal random $\varepsilon$-walks starting at $p$, i.e. continuous mappings $\sigma:[0, n \varepsilon] \rightarrow \mathbb{R}^{2}$ which begin at $\sigma(0)=p$ and for any integer $k=0, \ldots, n-1$,

$$
\left(\left.\sigma\right|_{[k \varepsilon,(k+1) \varepsilon]}\right)(t)=\sigma(k \varepsilon) \pm t e_{i},
$$

\footnotetext{
${ }^{6}$ Classically, a Riemann surface without boundary is called hyperbolic if it carries a nonconstant positive superharmonic function, and parabolic if it is neither elliptic (i.e. compact) nor hyperbolic. The reader should be aware that we will use the concept of parabolicity for Riemannian manifolds with boundary (see Definition 15) and reserve the word recurrent for manifolds without boundary (Definition 16). For Riemannian manifolds, the relationship between parabolicity and recurrence will become clear soon.
} 
where $e_{i}$ is one of the unit vectors $(1,0),(0,1)$. We define $\mu_{p}(n, \varepsilon)(I)$ to be the probability that some $\sigma \in \Gamma(p, n, \varepsilon)$ crosses $\partial \Omega$ a first time in $I$. As $n \rightarrow \infty$, $\mu_{p}(n, \varepsilon)(I)$ converges quickly to a number $\mu_{p}(\varepsilon)(I) \in[0,1]$. Similarly, as $\varepsilon \rightarrow 0$, the $\mu_{p}(\varepsilon)$ converge to a measure $\mu_{p}$ on $\partial M$, which is equal to the hitting measure obtained from Brownian motion starting at $p$.

For an interval $I \subset \partial \Omega$, consider the function $P_{I}(n, \varepsilon): \operatorname{Int}(\Omega) \rightarrow[0,1]$ defined as $P_{I}(n, \varepsilon)(p)=\mu_{p}(n, \varepsilon)(I)$ for $p \in \operatorname{Int}(\Omega)$. Note that for any $p \in \operatorname{Int}(\Omega)$ and for $\varepsilon$ smaller that the distance from $p$ to $\partial \Omega$, the following formula holds

$$
P_{I}(n, \varepsilon)(p)=\frac{1}{4}\left(\sum_{i=1}^{2} P_{I}(n-1, \varepsilon)\left(p+e_{i}\right)+\sum_{i=1}^{2} P_{I}(n-1, \varepsilon)\left(p-e_{i}\right)\right),
$$

and so, the limiting function $P_{I}(\varepsilon)$ satisfies an infinitesimal 4 point mean value property. As $\varepsilon \rightarrow 0, P_{I}(\varepsilon)$ converges to a function $P_{I}$ on $\operatorname{Int}(\Omega)$ which satisfies the usual mean value property. Therefore, the function $p \mapsto P_{I}(p)$, which is the probability of a Brownian path starting at $p$ of exiting $\Omega$ a first time on $I$, is a harmonic function which takes its values in $[0,1]$. Note that $P_{I}$ has limiting values 1 on the interior of $I$ and 0 on the interior of $\partial \Omega-I$ and so, it is the unique bounded harmonic function on $\operatorname{Int}(\Omega)$ whose boundary values correspond almost everywhere to the characteristic function of $I \subset \partial \Omega$. By definition of $P_{I}$, the hitting measure is $\mu_{p}(I)=P_{I}(p)$, which gives the desired equivalence between hitting and harmonic measure for planar domains.

The above discussion generalizes easily to a Riemannian manifold $M$ with boundary. We will briefly explain this generalization in the case of dimension 2 and when $M$ lies in the interior of a bigger complete manifold $\bar{M}$. Let $M$ be a Riemannian surface with boundary and $p \in \operatorname{Int}(M)$. Given a unit tangent vector $v_{p}, n \in \mathbb{N}$ and $\varepsilon>0$, we let $\Gamma\left(v_{p}, n, \varepsilon\right)$ denote the set of $n$-step orthogonal random $\varepsilon$-walks $\sigma:[0, n \varepsilon] \rightarrow \bar{M}$ such that $\left.\sigma\right|_{[0, \varepsilon]}$ is the unit speed geodesic beginning at $p$ in one of the directions $\pm v_{p}, \pm J v_{p}$, where $J$ is a local almost complex structure in a neighborhood of $p$, and $\left.\sigma\right|_{[k \varepsilon,(k+1) \varepsilon]}$ is the unit speed geodesic in $\bar{M}$ beginning at $\sigma(k \varepsilon)$ in one of the directions $\pm \sigma^{\prime}(k \varepsilon), \pm J \sigma^{\prime}(k \varepsilon), 0 \leq k \leq n-1$. These sets of random walks produce, as in the planar domain case, a limiting hitting measure on $\partial M$, which is independent of the initial choice of $v_{p}$, and by the previous arguments, is equal to the harmonic measure $\mu_{p}$.

From the above discussion, it easily follows that a Riemannian manifold without boundary is recurrent (see Definition 16) precisely when almost all Brownian paths are dense in the manifold. Also, parabolicity and harmonic measure are closely related, as states the following result.

Proposition 2. Let $(M, g)$ be a Riemannian manifold with $\partial M \neq \varnothing$. Then, the following statements are equivalent:

1. $M$ is parabolic.

2. There exists a point $p \in \operatorname{Int}(M)$ such that the harmonic measure $\mu_{p}$ is full, i.e. $\int_{\partial M} \mu_{p}=1$.

3. Given any $p \in \operatorname{Int}(M)$ and any bounded harmonic function $f: M \rightarrow \mathbb{R}$, then $f(p)=\int_{\partial M} f \mu_{p}$.

4. There exists a proper nonnegative superharmonic function on $M$.

$\mathbb{R}^{n}$ is recurrent for Brownian motion if and only if $n \leq 2$. The parabolicity of a Riemannian manifold with boundary is not affected by adding compact sets or by 
removing interiors of compact sets, and if a manifold $M$ can be decomposed as the union of two parabolic domains with compact intersection, then $M$ is parabolic (or recurrent, depending on if $\partial M$ is empty or not).

Note that if $h: M \rightarrow \mathbb{R}$ is a nonconstant positive harmonic function on a recurrent Riemannian manifold, then for any positive regular value $t \in \mathbb{R}$ of $h$, the closed subset $M_{t}=h^{-1}((0, t])$ is parabolic and $\left.h\right|_{M_{t}}$ is a bounded harmonic function with constant boundary value $t$. By Proposition $2,\left.h\right|_{M_{t}}$ is the constant function $t$, which contradicts that $t$ is a regular value of $h$. This contradiction completes the proof of the following well-known result.

Proposition 3 (Liouville Theorem). Every positive harmonic function on a recurrent Riemannian manifold is constant.

3.2. Recent results on parabolicity for minimal surfaces. As we have said before in this survey, a knowledge of the conformal type of a minimal surface $M$ is crucial when tackling uniqueness questions. Sometimes it is useful to decompose $M$ in pieces and study the conformal structure of each piece as a Riemann surface with boundary. For instance, the proof by Meeks and Rosenberg of the uniqueness of the helicoid [80] uses the fact that a simply connected properly embedded minimal surface $M \subset \mathbb{R}^{3}$ must admit a plane which intersects $M$ transversely in a single proper arc $\gamma$. Each of the two closed complements of $\gamma$ in $M$ is contained in a closed halfspace, hence both are parabolic as follows from Theorem 12 below and ones then proves that $M$ is conformally $\mathbb{C}$. This argument introduces one of the main open questions concerning minimal surfaces with boundary, which we now state after a definition.

Definition 17. Let $W \subset \mathbb{R}^{3}$ be a connected region of space which is either open or the closure of an open set. We say that $W$ is a universal region for surfaces if every complete, connected, properly immersed minimal surface $M \subset W$ is either recurrent (when $\partial M=\varnothing$ ) or a parabolic surface with boundary. $W$ is called a universal region for graphs if every proper minimal graph $M \subset W$ is a parabolic surface with boundary.

QUESTION 1. Which regions $W \subset \mathbb{R}^{3}$ are universal for surfaces or for graphs?

Obviously, any universal region for surfaces is also universal for graphs. By an ingenious application of the classical Runge's Theorem, Rosenberg and Toubiana [115] gave an example of a nonflat minimal annulus without boundary which is properly immersed in an open slab. By Liouville's Theorem (Proposition 3), this example proves that an open slab is not universal for surfaces. A smart refinement of the ideas used by Nadirashvili [94] in his proof of the existence of a complete immersed minimal surface in a ball in $\mathbb{R}^{3}$, allows one to construct a minimal immersion of the open unit disk that is proper in $\mathbb{R}^{3}$ (Morales $[\mathbf{9 2}]$ ), which shows that $\mathbb{R}^{3}$ is not universal for surfaces. This result demonstrates the necessity of the embeddedness assumption in Meeks and Rosenberg's proof that if a simply connected, proper minimal surface is embedded, then it will be conformally $\mathbb{C}$. Recently, Martín and Morales [65] have constructed a complete conformal minimal immersion of the open unit disk that is proper in an open ball, which implies that open balls are not universal regions for surfaces. More recently [64], they have generalized their result to prove that the interior of any convex region of $\mathbb{R}^{3}$ (including noncompact and nonsmooth ones) admits a proper complete minimal immersion of the unit disk, which implies that such regions are not universal for surfaces. Theorem 12 
and Corollary 1 below imply respectively that any closed halfspace is universal for surfaces, and the region above a negative halfcatenoid is universal for graphs.

Part 5 in Proposition 2 gives a method for showing that a given region $W \subset \mathbb{R}^{3}$ is universal for surfaces, which consists of finding a proper nonnegative universal superharmonic function on $W$, a concept that we now define.

Definition 18. Given a region $W \subset \mathbb{R}^{3}$, a function $h: W \rightarrow \mathbb{R}$ is said to be a universal superharmonic function on $W$ if its restriction to any minimal surface $M \subset W$ is superharmonic.

Examples of universal superharmonic functions on all of $\mathbb{R}^{3}$ include coordinate functions such as $x_{1}$ or the function $-x_{1}^{2}$. Collin, Kusner, Meeks and Rosenberg proved the useful inequality (Lemma 2.2 in [26]) valid for any immersed minimal surface in $\mathbb{R}^{3}$ :

$$
|\Delta \ln r| \leq \frac{\left|\nabla x_{3}\right|^{2}}{r^{2}}
$$

where $r=\sqrt{x_{1}^{2}+x_{2}^{2}}$ and $\nabla, \Delta$ denote the intrinsic gradient and laplacian on $M$. Using the estimate (11), it is straightforward to check the following statement.

LEMMA 1.

i) The function $\ln r-x_{3}^{2}$ is a universal superharmonic function in the region $\left\{r^{2} \geq \frac{1}{2}\right\}$.

ii) The function $\ln r-x_{3} \arctan x_{3}+\frac{1}{2} \ln \left(x_{3}^{2}+1\right)$ is a universal superharmonic function in the region $\left\{r^{2} \geq x_{3}^{2}+1\right\}$.

With the above Lemma, we now prove that any closed halfspace is a universal region for surfaces. If $M$ is a properly immersed minimal surface in a closed halfspace and $\partial M=\varnothing$, then $M$ is planar by the Halfspace Theorem (Theorem 7 ); in particular, any closed halfspace is a universal region for surfaces without boundary. The desired property of being planar also follows directly from the following general Theorem and the fact that on recurrent surfaces positive harmonic functions are constant (Proposition 3).

Theorem 12 (Collin, Kusner, Meeks, Rosenberg [26]). Let $M$ be a connected properly immersed minimal surface in $\mathbb{R}^{3}$, possibly with boundary. Then, every component of the intersection of $M$ with a closed halfspace is a parabolic surface with boundary. In particular, if $M$ has empty boundary and intersects some plane in a compact set, then $M$ is recurrent.

Proof. Up to a rotation, it suffices to check that any component $C$ of $M(+)=$ $M \cap\left\{x_{3} \geq 0\right\}$ is parabolic. For fixed $n \in \mathbb{N}$, let $C_{n}=C \cap x_{3}^{-1}([0, n])$. By part i) of Lemma 1 , the function $h=\ln r-x_{3}^{2}$ is superharmonic and proper when restricted to $C_{n} \cap\left\{r^{2} \geq \frac{1}{2}\right\}$. Furthermore, $h$ is positive outside a compact domain on $C_{n}$, which by part 4 of Proposition 2 implies that $C_{n} \cap\left\{r^{2} \geq \frac{1}{2}\right\}$ is parabolic. Since $M$ is proper and $\left\{r^{2} \leq \frac{1}{2}\right\} \cap\left\{0 \leq x_{3} \leq n\right\}$ is compact, we deduce that $C_{n}-\left\{r^{2} \geq \frac{1}{2}\right\}$ is a compact subset of $C_{n}$. Since parabolicity is not affected by adding compact subsets, it follows that $C_{n}$ is parabolic.

We now check that $C$ is parabolic. Fix a point $p \in C$ with $x_{3}(p)>0$ and let $\mu_{p}^{C}$ be the harmonic measure of $C$ with respect to $p$. For $n$ large enough, $p$ lies in the interior of $C_{n}$. Since $x_{3}$ is a bounded harmonic function on the parabolic surface 
$C_{n}$, part 3 of Proposition 2 insures that

$$
x_{3}(p)=\int_{\partial C_{n}} x_{3} \mu_{p}^{n} \geq n \int_{\partial C_{n} \cap x_{3}^{-1}(n)} \mu_{p}^{n},
$$

where $\mu_{p}^{n}$ is the harmonic measure of $C_{n}$ with respect to $p$. Since $\mu_{p}^{n}$ is full on $\partial C_{n}$, it follows that

$$
\int_{\partial C_{n}-x_{3}^{-1}(n)} \mu_{p}^{n}=1-\int_{\partial C_{n} \cap x_{3}^{-1}(n)} \mu_{p}^{n} \geq 1-\frac{x_{3}(p)}{n} \stackrel{(n \rightarrow \infty)}{\longrightarrow} 1 .
$$

Suppose that $M$ and $N$ are Riemannian manifolds with $M \subset N, \partial$ a component of $\partial M \cap \partial N, p \in \operatorname{Int}(M)$ and $\mu_{p}^{M}$ and $\mu_{p}^{N}$ are the respective harmonic measures. Then it follows immediately from the definition of harmonic measure that $\int_{\partial} \mu_{p}^{M} \leq$ $\int_{\partial} \mu_{p}^{N} \leq 1$. By letting $M=C_{n}, N=C$ and $\partial=\partial C_{n}-x_{3}^{-1}(n)$, the above displayed inequality implies $\lim _{n} \int_{\partial C_{n}-x_{3}^{-1}(n)} \mu_{p}^{C} \geq 1$, from where we conclude that $\int_{\partial C} \mu_{p}^{C}=1$ and the proof is complete.

An open subset $\Omega$ of a Riemann surface without boundary is called hyperbolic if $\Omega$ carries a nonconstant positive superharmonic function, or equivalently if for any point $q \in \Omega$, the Green's function with singularity at $q$ exists (this function is the smallest positive harmonic function in $\Omega-\{q\}$ with a logarithmic singularity at $q$, see [32] for details about Green's functions and hyperbolicity). Suppose now that $X: M \rightarrow \mathbb{R}^{3}$ is a proper minimal immersion whose Gauss map $g$ has image set contained in a hyperbolic open subset $\Omega \subset \mathbb{S}^{2}$. Since such an $\Omega$ does not have logarithmic capacity zero, a result by Osserman [99] implies that if $M$ has no boundary, then the immersion is flat. Assume that $X$ is not flat. A careful analysis of the role that $x_{3}$ plays in the last proof lets us exchange this coordinate function by the composition $G \circ g$, where $G$ is the Green's function in $\Omega$ with singularity at a given point $q \in \Omega$. After a suitable choice of a universal superharmonic function $h$ which constrains the region where $X(M)$ is contained, arguments not too different from the ones in the proof of Theorem 12 lead to the following statement.

Theorem 13 (López, Pérez [62]). Given $\alpha \in(0,1)$, let $W_{\alpha}=\left\{x_{3}>-\left(x_{1}^{2}+\right.\right.$ $\left.\left.x_{2}^{2}\right)^{\alpha / 2}\right\}$ and $X: M \rightarrow \mathbb{R}^{3}$ a proper nonflat minimal immersion with $X(M) \subset W_{\alpha}$. If, up to removing a compact set of $M$, the Gauss map of $X$ has image contained in a hyperbolic open subset of the sphere, then $M$ is a parabolic surface with nonempty boundary.

The region above a vertical negative halfcatenoid and outside a certain compact set is contained in $W_{\alpha}$ for any $\alpha \in(0,1)$. Since the Gauss map image of a graph is contained in a closed halfsphere, it is also contained in an hyperbolic open subset of $\mathbb{S}^{2}$. Thus the following result is a direct consequence of Theorem 13 .

COROLlaRY 1. Any proper minimal graph lying in the closed region above a vertical negative halfcatenoid is parabolic.

We would like to finish this Section by mentioning an unpublished example by Collin of a complete stable minimal submersion of a disk minus a Cantor set of positive measure in its boundary into a plane, which we can view as a degenerate multigraph.

Let $W=\mathbb{R}^{2}-\{0,1\}$ and let $h: \mathbb{D} \rightarrow W$ be the universal cover by the open unit disk $\mathbb{D} \subset \mathbb{C}$. Note that $\mathbb{D}$ is incomplete in the pulled-back flat metric through $h$. 
Consider the simply connected subdomain $W(+)=\left\{\left(x_{1}, x_{2}\right) \in W \mid x_{2} \geq 0\right\}$ and one of its lifts $D_{1}$ to $\mathbb{D}$. Let $p_{1}, q_{1}$ denote points in $\partial \mathbb{D}$ corresponding to the end points of $D_{1}$, which under completion would map to 0 and 1 in $\mathbb{R}^{2}$, respectively. Consider small disjoint arcs $\alpha_{1}, \beta_{1}$ in $\partial \mathbb{D}$ centered at $p_{1}, q_{1}$ respectively and each of length $l(1)<\frac{1}{16}$. Consider the open "equilateral" triangles $\Delta(1,1), \Delta(1,2)$ in $\mathbb{D}$ with circle bases $\alpha_{1}, \beta_{1}$, and sides being straight line segments of length $l(1)$. Let $D_{1}, D_{2}, \ldots, D_{n}, \ldots$ denote an ordering of the set of lifts of $W(+)$ to $\mathbb{D}$. For each $D_{k}$ we find similar points $p_{k}, q_{k}$ and $\operatorname{arcs} \alpha_{k}, \beta_{k}$ with lengths at most $l(k)<\frac{1}{16^{k}}$. After removing from $\mathbb{D}$ all of the similarly defined open "equilateral" triangles $\Delta(k, 1), \Delta(k, 2)$, one obtains a flat surface $M$ and an induced map $h: M \rightarrow \mathbb{R}^{2}$. Since the boundary of the closure $\bar{M}$ of $M$ in the closed unit disk $\overline{\mathbb{D}}$ is a Lipschitz curve parametrized by the argument $\theta$, the Riemann mapping Theorem preserves sets of positive Lebesgue measure on $\partial \bar{M}$. By construction, the set $\partial \bar{M}-\partial M$ has positive measure and so $\partial M$ does not have full harmonic measure. On the other hand, it can be checked that the flat metric on $M$ induced by the submersion is complete, which finishes our construction of the example of Collin.

\section{Stable minimal surfaces.}

Very often, stable minimal surfaces play the role of planes separating disjoint pieces of minimal surfaces. How to produce such separating stable surfaces will be the goal of Subsection 4.2. Before explaining this, we will show how to give bounds on the area and curvature for stable surfaces, results which in turn have important consequences to the global theory.

4.1. Area and curvature estimates for stable minimal surfaces. Let $D \subset M$ be an embedded geodesic disk of radius $r_{0}>0$ contained in a minimal surface $M \subset \mathbb{R}^{3}$. Since the Gaussian curvature of $D$ is nonpositive, classical comparison with the Euclidean disk of the same radius gives $\pi r_{0}^{2} \leq \operatorname{Area}(D)$. Colding and Minicozzi (Theorem 1.2 in [22]) gave the following useful upper estimate for Area $(D)$ by assuming stability.

THEOREM 14. Let $D \subset M$ be a geodesic disk of radius $r_{0}$ inside a minimal surface $M \subset \mathbb{R}^{3}$. If $D$ is stable, then

$$
\operatorname{Area}(D) \leq \frac{4}{3} \pi r_{0}^{2}
$$

Proof. The argument of Colding-Minicozzi uses a beautiful application of the stability inequality with a particular choice of a radial test function $f(r, \theta)=\eta(\theta)$ (here $(r, \theta)$ are polar geodesic coordinates on $D)$, where $\eta \in C^{1}\left(\left[0, r_{0}\right]\right)$ with $\eta\left(r_{0}\right)=$ 0 . By stability, Green's formula and the coarea formula, we obtain:

$$
0 \leq \int_{D}\left(|\nabla f|^{2}+2 K f^{2}\right) d A=\int_{0}^{r_{0}}\left(\eta^{\prime}(r)\right)^{2} l(r) d r+2 \int_{0}^{r_{0}}\left(\int_{\partial D(r)} K d s_{r}\right) \eta^{2}(r) d r
$$

where $K$ is the Gaussian curvature function on $M, D(r)$ is the disk of geodesic radius $r \in\left[0, r_{0}\right]$ concentric with $D$ and $d s_{r}, l(r)$ are respectively the length element and the total length of $\partial D(r)$. Let $K(r)=\int_{D(r)} K d A$. As $K^{\prime}(r)=\int_{\partial D(r)} K d s_{r}$ and $K(0)=\eta\left(r_{0}\right)=0$, integration by parts gives $\int_{0}^{r_{0}}\left(\int_{\partial D(r)} K d s_{r}\right) \eta^{2}(r) d r=$ $-\int_{0}^{r_{0}} K(r)\left(\eta^{2}(r)\right)^{\prime} d r$. By the Gauss-Bonnet formula and the first variation of 
length (see e.g. $[\mathbf{1 0}]), K(r)=2 \pi-\int_{\partial D(r)} \kappa_{g} d s_{r}=2 \pi-l^{\prime}(r)$, where $\kappa_{g}$ denotes geodesic curvature. Substituting this formula for $K(r)$ into (12), we obtain:

$$
0 \leq \int_{0}^{r_{0}}\left(\eta^{\prime}(r)\right)^{2} l(r) d r-2 \int_{0}^{r_{0}}\left(2 \pi-l^{\prime}(r)\right)\left(\eta^{2}(r)\right)^{\prime} d r .
$$

Now taking $\eta(r)=1-\frac{r}{r_{0}}$ in the last expression, we have

$$
-\frac{1}{r_{0}^{2}} \int_{0}^{r_{0}} l(r) d r+\frac{4}{r_{0}} \int_{0}^{r_{0}} l^{\prime}(r)\left(1-\frac{r}{r_{0}}\right) d r \leq \frac{8 \pi}{r_{0}} \int_{0}^{r_{0}}\left(1-\frac{r}{r_{0}}\right) d r=4 \pi .
$$

Finally, integration by parts gives $\frac{4}{r_{0}} \int_{0}^{r_{0}} l^{\prime}(r)\left(1-\frac{r}{r_{0}}\right) d r=\frac{4}{r_{0}^{2}} \int_{0}^{r_{0}} l(r) d r$. Plugging this into (13) and using that $\int_{0}^{r_{0}} l(r) d r=\operatorname{Area}(D)$, we finish the proof.

To see the usefulness of the above area estimate, we now give a short proof of Theorem 8.

Proof of Theorem 8 . Let $M \subset \mathbb{R}^{3}$ be a complete orientable immersed minimal surface which is stable. Recall that for orientable minimal surfaces, stability is equivalent to the existence of a positive Jacobi function (Proposition 1 in [34]). After lifting such a function on $M$ to a Jacobi function on the universal cover of $M$, we can assume that $M$ is simply-connected. Since the Gaussian curvature of $M$ is nonpositive, Hadamard's Theorem implies that the intrinsic distance function to a given point $p_{0} \in M$ is smooth outside $p_{0}$ without critical points and that the geodesic disk $D(r)$ centered at $p_{0}$ with radius $r>0$ is embedded. Let $A(r)$ be the area of $D(r)$ (which is a smooth function of $r$ ) and $l(r)$ the length of its boundary. Then, $A^{\prime}(r)=l(r)$ and thus, the first variation of length and the Gauss-Bonnet formula give $A^{\prime \prime}(r)=\int_{\partial D(r)} \kappa_{g} d s_{r}=2 \pi-\int_{D(r)} K d A$, where $\kappa_{g}$ is the geodesic curvature of $\partial D(r), d s_{r}$ is its length element and $K$ is the Gaussian curvature of $M$. The last equality implies that $A^{\prime \prime}(r)$ is monotonically increasing in $r$. This property together with $A(r) \leq \frac{4}{3} \pi r^{2}$ (Theorem 14) imply that $A^{\prime \prime}(r) \leq \frac{8}{3} \pi$, which shows that $-\int_{D(r)} K d A \leq \frac{2}{3} \pi$. Since $r$ is arbitrary, we conclude that $M$ has finite total absolute Gaussian curvature at most $\frac{2}{3} \pi$. By Theorem 2, the total curvature of a complete orientable nonplanar minimal surface is infinite or a positive integer multiple of $-4 \pi$, and so we deduce that $M$ must be a plane. This finishes the proof of Theorem 8.

A crucial fact in minimal surface theory is that orientable minimally immersed stable surfaces with boundary in $\mathbb{R}^{3}$ have curvature estimates up to their boundary. These curvature estimates were firstly obtained by Schoen [117] and later proved in another way by Ros [113]. Here we give a different approach, using Theorem 8 and a blow-up argument. As a consequence, these curvature estimates are in fact easily derived from the area estimate in Theorem 14.

THEOREM 15. There exists a universal constant $c>0$ such that for any stable orientable minimally immersed surface $M$ in $\mathbb{R}^{3}$, its absolute curvature function times the squared distance function to the boundary of $M$ is bounded above by $c$.

Proof. Suppose on the contrary that there exist stable orientable minimally immersed surfaces $M_{n}$ in $\mathbb{R}^{3}$ and points $p_{n} \in M_{n}$ in the interior of such surfaces such that $\left|K_{M_{n}}\right|\left(p_{n}\right) d_{M_{n}}\left(p_{n}, \partial M_{n}\right)^{2}>n$ for all $n$, where $K_{M_{n}}, d_{M_{n}}$ are respectively the Gaussian curvature and the intrinsic distance in $M_{n}$. By passing to the universal covering, we may assume that $M_{n}$ is simply connected for each $n$. Let $D_{n}$ be 
the embedded geodesic disk centered at $p_{n}$ with radius $d_{M_{n}}\left(p_{n}, \partial M_{n}\right)$ and $q_{n} \in$ $D_{n}$ a point where the function $d_{M_{n}}\left(\cdot, \partial D_{n}\right)^{2}\left|K_{M_{n}}\right|: D_{n} \rightarrow[0, \infty)$ has a maximum value. We denote by $\widetilde{D}_{n}$ the minimal disk in $\mathbb{R}^{3}$ obtained by first translating by $-q_{n}$ the intrinsic disk in $M_{n}$ with center $q_{n}$ and radius $\frac{1}{2} d_{M_{n}}\left(q_{n}, \partial D_{n}\right)$ and then homothetically expanding this translated disk by the scaling factor $\sqrt{\left|K_{M_{n}}\left(q_{n}\right)\right|}$. Thus we have a sequence $\left\{\widetilde{D}_{n}\right\}_{n}$ of orientable stable minimal disks in $\mathbb{R}^{3}$, all passing through the origin with $d_{\widetilde{M}_{n}}\left(0, \partial \widetilde{D}_{n}\right)^{2} \geq n$ and with Gaussian curvature function $K_{\widetilde{D}_{n}}$ satisfying $K_{\widetilde{D}_{n}}(0)=-1$ and $K_{\widetilde{D}_{n}} \geq-4$ on $\widetilde{D}_{n}$. A standard compactness result (see for example [80] or [107]) shows that a subsequence of the $\widetilde{D}_{n}$ converges uniformly on compact sets of $\mathbb{R}^{3}$ to an orientable, complete, simply-connected, immersed minimal surface $\widetilde{D}_{\infty}$ passing through the origin, with bounded Gaussian curvature $K_{\widetilde{D}_{\infty}}, K_{\widetilde{D}_{\infty}}(0)=-1$ and empty boundary. Since $\widetilde{D}_{\infty}$ is stable (because the smooth limit of stable minimal surfaces is stable), Theorem 8 implies that $\widetilde{D}_{\infty}$ is a plane, which is a contradiction. This finishes our proof of Theorem 15.

REMARK 1. Another blow-up argument implies that Theorem 15 also holds for stable minimal surfaces in a Riemannian three-manifold $N^{3}$ with injectivity radius bounded from below and which is uniformly locally quasi-isometric to balls in Euclidean space (in particular, it holds on any compact $N^{3}$ ).

4.2. Barrier constructions. Barrier constructions allow one to construct compact and noncompact stable minimal surfaces in $\mathbb{R}^{3}$ that are constrained to lie in subdomains of $\mathbb{R}^{3}$ whose boundaries have nonnegative mean curvature. For example, consider two connected properly embedded disjoint minimal surfaces $M_{1}, M_{2}$ in $\mathbb{R}^{3}$ and the closed connected region $W$ of $\mathbb{R}^{3}$ with $\partial W=M_{1} \cup M_{2}$. We now show how to produce compact stable embedded minimal surfaces in $W$. First note that $W$ is a complete flat manifold with boundary having zero mean curvature. Meeks and Yau [89] proved that $W$ embeds isometrically in a complete homogeneously regular ${ }^{7}$ Riemannian manifold $\widetilde{W}$ diffeomorphic to the interior of $W$ and with metric $g$. Morrey $[\mathbf{9 3}]$ proved that in a homogeneously regular three-manifold one can solve the classical Plateau problem or other area minimizing problems. In particular, if $\Gamma$ is an embedded 1-cycle in $\widetilde{W}$ which bounds an orientable chain in $\widetilde{W}$, then $\Gamma$ is the boundary of a compact least area embedded surface $\Sigma_{\Gamma}(g) \subset \widetilde{W}$. Meeks and Yau prove that their metric $g$ on $\widetilde{W}$ can be approximated by a family of homogeneously regular metrics $\left\{g_{n}\right\}_{n \in \mathbb{N}}$ which converge smoothly on compact subdomains to $g$ and each $g_{n}$ satisfies a convexity condition outside of $W \subset \widetilde{W}$, which forces the least area surface $\Sigma_{\Gamma}\left(g_{n}\right)$ to lie in $W$ if $\Gamma$ lies in $W$. A subsequence of the $\Sigma_{\Gamma}\left(g_{n}\right)$ converges to a smooth minimal surface $\Sigma_{\Gamma}$ of least area in $W$ with respect to the original flat metric. We now use this barrier construction to prove the Strong Halfspace Theorem (also see Theorem 7).

TheOREM 16. $[\mathbf{5 0 , 8 5}]$ If $M_{1}$ and $M_{2}$ are two disjoint properly immersed minimal surfaces in $\mathbb{R}^{3}$, then $M_{1}$ and $M_{2}$ are parallel planes.

${ }^{7}$ A complete Riemannian manifold $(\widetilde{W}, g)$ is homogeneously regular if there exists an $\varepsilon>0$ such that the injectivity radius of $\widetilde{W}$ is at least $\varepsilon$ and $\varepsilon$-balls in $\widetilde{W}$ are uniformally quasi-isometric to $\varepsilon$-balls in $\mathbb{R}^{3}$. For example, every compact Riemannian three-manifold satisfies this rather weak property. 
Proof. Let $W$ be the closed complement of $M_{1} \cup M_{2}$ in $\mathbb{R}^{3}$ that has portions of both $M_{1}$ and $M_{2}$ on its boundary. The surface $\partial W$ is a good barrier for solving Plateau-type problems in $W$. Let $M_{1}(1) \subset \ldots \subset M_{1}(n) \subset \ldots$ be a compact exhaustion of $M_{1}$ and let $\Sigma_{1}(n)$ be a least area surface in $W$ with boundary $\partial M_{1}(n)$. Let $\alpha$ be a compact arc in $W$ which joins a point in $M_{1}(1)$ to a point in $\partial W \cap M_{2}$. By elementary intersection theory, $\alpha$ intersects every least area surface $\Sigma_{1}(n)$. By compactness of least area surfaces, a subsequence of the surfaces $\Sigma_{1}(n)$ converges to a properly embedded area minimizing surface $\Sigma$ in $W$ with a component $\Sigma_{0}$ which intersects $\alpha$. Since $\Sigma_{0}$ separates $\mathbb{R}^{3}, \Sigma_{0}$ is orientable and so by Theorem $8, \Sigma_{0}$ is a plane. Hence, $M_{1}$ and $M_{2}$ lie in closed halfspaces of $\mathbb{R}^{3}$ and so, by Theorem $12, M_{1}$ and $M_{2}$ are recurrent. But, then the height of $M_{1}, M_{2}$ over their separating plane is a positive harmonic function which must be constant (Proposition 3). Hence, $M_{1}$ and $M_{2}$ must be planes (instead of using Theorem 12, one could apply the Halfspace Theorem (Theorem 7 ) to conclude that $M_{1}$ and $M_{2}$ are parallel planes.)

Another useful application of the barrier construction is the following. Suppose $\Gamma$ is an extremal simple closed curve in $\mathbb{R}^{3}$, i.e. $\Gamma$ lies on the boundary of its convex hull $B$. We first assume that $\partial B$ is smooth. By the Jordan curve Theorem, $\Gamma$ is the boundary of two disks $D_{1}, D_{2} \subset \partial B$. Assume $\Gamma$ bounds two different branched minimal immersions and let $\Sigma$ denote their union. Let $W_{1}, W_{2}$ be the geodesic completions of the two components of $B-\Sigma$ which contain the disks $D_{1}, D_{2}$. In this case $\partial W_{1}$ and $\partial W_{2}$ consist of smooth pieces with zero mean curvature and convex corners. Meeks and Yau [89] proved that such boundaries are good barriers for solving least area problems. In fact, in this case they prove that $\Gamma$ bounds a least area embedded disk $\widetilde{D}_{1} \subset W_{1}$ and a different least area embedded disk $\widetilde{D}_{2} \subset W_{2}$. Similarly, if $\Gamma$ bounds a unique branched minimal surface which is not an embedded stable minimal disk, then with this barrier argument we produce two different embedded minimal disks with boundary $\Gamma$, which is a contradiction. If $\partial B$ is not assumed to be smooth, then one can use an approximation argument by convex smooth boundaries (see e.g. $[\mathbf{8 8}]$ ) to have the same conclusion.

On the other hand, Nitsche $[\mathbf{9 6}]$ proved that a regular analytic Jordan curve in $\mathbb{R}^{3}$ whose total curvature is at most $4 \pi$ bounds a unique minimal disk. The hypothesis of analyticity for the boundary curve in Nitsche's Theorem comes from consideration of boundary branch points. When $\Gamma$ is $C^{2}$ and extremal, there are never boundary branch points as shown in [89].

THEOREM 17. [89] If $\Gamma$ is a $C^{2}$-extremal curve with total curvature at most $4 \pi$, then $\Gamma$ is the boundary of a unique compact branched minimal surface and this surface is a smooth embedded minimal disk of least area.

\section{The Ordering Theorem for the ends of properly embedded minimal surfaces.}

The study of the ends of a properly embedded minimal surface $M \subset \mathbb{R}^{3}$ with more than one end has been extensively developed. Callahan, Hoffman and Meeks [7] showed that in one of the closed complements of $M$ in $\mathbb{R}^{3}$ there exists a noncompact properly embedded minimal surface $\Sigma \subset \mathbb{R}^{3}-M$ with compact boundary and finite total curvature. By the discussion following Theorem 2, the ends of $\Sigma$ are of catenoidal or planar type, and the embeddedness of $\Sigma$ forces its ends to have parallel normal vectors at infinity. 
DEFINITION 19. In the above situation, the limit tangent plane at infinity of $M$ is the plane in $\mathbb{R}^{3}$ passing through the origin whose normal vector equals (up to sign) the limiting normal vector at the ends of $\Sigma$. Such a plane is unique [7], in the sense that it does not depend on the finite total curvature minimal surface $\Sigma \subset \mathbb{R}^{3}-M$.

The limit tangent plane at infinity is a key notion for studying the way in which a minimal surface with more than one end embeds properly in space.

TheOREM 18 (Ordering Theorem [39]). Let $M \subset \mathbb{R}^{3}$ be a properly embedded minimal surface with more than one end and horizontal limit tangent plane at infinity. Then, the space $\mathcal{E}(M)$ of ends of $M$ is linearly ordered geometrically by the relative heights of the ends over the $\left(x_{1}, x_{2}\right)$-plane, and embeds topologically in $[0,1]$ in an ordering preserving way. Furthermore, this ordering has a topological nature in the following sense: If $M$ is properly isotopic to a properly embedded minimal surface $M^{\prime}$ with horizontal limit tangent plane at infinity, then the associated ordering of the ends of $M^{\prime}$ either agrees with or is opposite to the ordering coming from $M$.

The linear ordering on the set of ends $\mathcal{E}(M)$ given by Theorem 18 lets us define the top end $e_{T}$ of $M$ as the unique maximal element in $\mathcal{E}(M)$ in the ordering (recall that $\mathcal{E}(M) \subset[0,1]$ is compact, hence $e_{T}$ exists). Analogously, the bottom end $e_{B}$ of $M$ is the unique minimal element in $\mathcal{E}(M)$. If $e \in \mathcal{E}(M)$ is neither the top nor the bottom end of $M$, then it is called a middle end of $M$.

Rather than sketching the proof of the Ordering Theorem, we will be content to explain how one obtains the linear ordering. Suppose $M \subset \mathbb{R}^{3}$ is a minimal surface in the hypotheses of Theorem 18 and let $\mathcal{A} \subset \mathcal{E}(M)$ be the set of annular ends of $M$. By Theorem 1.1 in [83], each end $e \in \mathcal{A}$ is either planar or its third coordinate function is proper (since we are assuming that the limit tangent plane at infinity of $M$ is horizontal). In this setting, Collin's Theorem (Theorem 3) insures that $e$ has finite total curvature and thus, it is asymptotic to a horizontal plane or to a halfcatenoid. Since the ends in $\mathcal{A}$ are all graphs over complements of compact subdomains in the $\left(x_{1}, x_{2}\right)$-plane, we see that $\mathcal{A}$ has a natural linear ordering by relative heights of its ends over the $\left(x_{1}, x_{2}\right)$-plane. Hence the Ordering Theorem is proved when $\mathcal{A}=\mathcal{E}(M)$.

By Theorem 9 , any end of $M$ which can be represented by a proper stable subdomain can be also represented by a surface of finite total curvature and so, it can be represented by an annulus. Let $e_{1}=\left[\alpha_{1}\right] \in \mathcal{E}(M)$ be an end which is not annular. Such an end can always be represented by a proper subdomain $E_{1}$ which is unstable and where $\partial E_{1}$ is connected and $\overline{M-E_{1}}$ is also unstable and noncompact (we are assuming $M$ has at least 2 ends). Let $W_{1}, W_{2}$ be the two closed complements of $M$ in $\mathbb{R}^{3}$. Note that we can consider $E_{1}$ to lie on the boundary of both of these complete flat 3-manifolds $W_{1}, W_{2}$, and that their boundaries $\partial W_{1}, \partial W_{2}$ are good barriers for solving Plateau-type problems. Since $E_{1}$ and $\overline{M-E_{1}}$ are both noncompact, elementary separation properties for surfaces in $\mathbb{R}^{3}$ imply that $\partial E_{1}$ is not homologous to zero in one of the domains $W_{1}, W_{2}$; suppose that $\partial E_{1}$ is not homologous to zero in $W_{1}$. Since $\partial E_{1}$ bounds the locally finite 2-chain $E_{1}$ in $\partial W_{1}$, the barrier argument in Subsection 4.2 shows that $\partial E_{1}$ is the boundary of a properly embedded orientable least area surface $\Sigma_{1}$ in $W_{1}$, which is noncompact since $\partial E_{1}$ is not homologous to zero in $W_{1}$. Similarly, $\partial E_{1}$ is the boundary of a 
least area (possibly compact) surface $\Sigma_{2}$ in $W_{2}$. Since $E_{1}$ and $M-E_{1}$ are unstable, the maximum principle implies that $\left(\Sigma_{1} \cup \Sigma_{2}\right) \cap M=\partial E_{1}$.

Let $R_{1}$ be the closed complement of $\Sigma_{1} \cup \Sigma_{2}$ in $\mathbb{R}^{3}$ which contains $E_{1}$ and let $R_{2}$ be the other closed complement. Since $\Sigma_{1}$ and $\overline{M-E_{1}}$ are both noncompact and $M$ is properly embedded in $\mathbb{R}^{3}, R_{1}$ and $R_{2}$ are both noncompact. It follows that outside a large ball containing $\partial E_{1}$, the boundary of $R_{1}$, which equals $\Sigma_{1} \cup \Sigma_{2}=$ $\partial R_{2}$, consists of a finite positive number of graphical ends which are asymptotic to the ends of horizontal planes and vertical catenoids. Let $e_{2}=\left[\alpha_{2}\right] \in \mathcal{E}(M)$ be an end with a representative $E_{2}$ which is disjoint from $E_{1}$ (note that any two distinct ends can be chosen to have disjoint representatives). The proper $\operatorname{arc} \alpha_{1}$ eventually lies in $R_{1}$ and so, it eventually lies between two successive graphical ends of $\partial R_{1}$ or $\alpha_{1}$ eventually lies in the region above the top graphical end of $\partial R_{1}$ or below the bottom graphical end of $\partial R_{1}$. A similar statement holds for the proper $\operatorname{arc} \alpha_{2} \subset R_{2}$. In particular there is a topological graphical plane $P$ over the $\left(x_{1}, x_{2}\right)$-plane whose end is one of the ends of $\partial R_{1}$ and eventually $\alpha_{1}$ and $\alpha_{2}$ lie on opposite sides of $P$. If $\alpha_{1}$ eventually lies below $P$ and $\alpha_{2}$ eventually lies above $P$, then $\left[\alpha_{1}\right]<\left[\alpha_{2}\right]$ in the linear ordering given by the Ordering Theorem; otherwise, $\left[\alpha_{2}\right]<\left[\alpha_{1}\right]$. The ordering we have just described can be proven to be a well-defined linear ordering, see $[\mathbf{3 9}]$ for more details.

\section{Quadratic area growth and recurrence.}

In this section we will sketch the proof of a Theorem that constrains both the geometry and the topology of properly embedded minimal surfaces in $\mathbb{R}^{3}$ with more than one end. This Theorem has been used in an essential way by Meeks, Perez and Ros in the proofs of their classification results in Theorem 29, Theorem 31 and Theorem 32 below, as well as by Frohman and Meeks in their proof of the Topological Classification of Minimal Surfaces (Theorem 28); all of these Theorems are discussed in Section 10 of this survey.

The Ordering Theorem in the previous Section represents the first step in trying to understand the geometry of properly embedded minimal surfaces with more than one end. By the proof of the Ordering Theorem, a middle end of a properly embedded minimal surface $M$ with horizontal limit tangent plane at infinity can be represented by a proper subdomain $E \subset M$ with compact boundary such that $E$ "lies between two catenoids." This means that $E$ is contained in a neighborhood $S$ of the $\left(x_{1}, x_{2}\right)$-plane, $S$ being topologically a slab, whose width grows at most logarithmically with the distance from the origin.

Suppose for the moment that $E$ is in fact contained in the region $W=\left\{\left(x_{1}, x_{2}, x_{3}\right)\right.$ $\left.\mid r \geq 1,0 \leq x_{3} \leq 1\right\}$, where $r=\sqrt{x_{1}^{2}+x_{2}^{2}}$. In Section 3 we defined the universal superharmonic function $\ln r-x_{3}^{2}$ in $W$. In particular, the restriction $f: E \rightarrow \mathbb{R}$ is superharmonic and proper. Suppose $f(\partial E) \subset[-1, c]$ for some $c>0$. Replace $E$ by $f^{-1}[c, \infty)$ and let $E(t)=f^{-1}[c, t]$ for $t>c$. Assuming that both $c, t$ are regular values of $f$, the Divergence Theorem gives

$$
\int_{E(t)} \Delta f d A=-\int_{f^{-1}(c)}|\nabla f| d s+\int_{f^{-1}(t)}|\nabla f| d s
$$

where $\nabla, \Delta$ are the intrinsic gradient and laplacian on $M$, and $d A, d s$ denote the corresponding area and length elements. 
Since $f$ is superharmonic, the function $t \mapsto \int_{E(t)} \Delta f d A$ is monotonically decreasing and bounded from below by $-\int_{f^{-1}(c)}|\nabla f| d s$. In particular, $\Delta f$ lies in $L^{1}(E)$. Furthermore, $|\Delta f|=\left.\left.|\Delta \ln r-2| \nabla x_{3}\right|^{2}|\geq-| \Delta \ln r|+2| \nabla x_{3}\right|^{2}$. By estimate (11) in Section 3, $|\Delta \ln r| \leq \frac{\left|\nabla x_{3}\right|^{2}}{r^{2}}$, hence $|\Delta f| \geq\left(2-\frac{1}{r^{2}}\right)\left|\nabla x_{3}\right|^{2}$. Since $r^{2} \geq 1$ in $W$, it follows $|\Delta f| \geq\left|\nabla x_{3}\right|^{2}$ and thus, both $\left|\nabla x_{3}\right|^{2}$ and $\Delta \ln r$ are in $L^{1}(E)$. This implies that outside of a subdomain of $E$ of finite area, $E$ can be assumed to be as close to being horizontal as one desires and in particular for the radial function $r$ on this horizontal part of $E,|\nabla r|$ is almost equal to 1 .

Let $r_{0}=\left.\max r\right|_{\partial E}$. With a slight abuse of notation, redefine $E(t)$ to be the subdomain of $E$ that lies inside the region $\left\{r_{0}^{2} \leq x_{1}^{2}+x_{2}^{2} \leq t^{2}\right\}$. Since $\int_{E(t)} \Delta \ln r d A=$ $-\int_{r=r_{0}} \frac{|\nabla r|}{r} d s+\int_{r=t} \frac{|\nabla r|}{r} d s=$ const. $+\frac{1}{t} \int_{r=t}|\nabla r| d s$ and $\Delta \ln r \in L^{1}(E)$, then the following limit exists:

$$
\lim _{t \rightarrow \infty} \frac{1}{t} \int_{r=t}|\nabla r| d s=C
$$

for some positive constant $C$. Thus, $t \mapsto \int_{r=t}|\nabla r| d s$ grows at most linearly as $t \rightarrow \infty$. By the coarea formula, for $t_{1}$ fixed and large

$$
\int_{E \cap\left\{t_{1} \leq r \leq t\right\}}|\nabla r|^{2} d A=\int_{t_{1}}^{t}\left(\int_{r=\tau}|\nabla r| d s\right) d \tau ;
$$

hence, $t \mapsto \int_{E \cap\left\{t_{1} \leq r \leq t\right\}}|\nabla r|^{2} d A$ grows at most quadratically as $t \rightarrow \infty$. Finally, since outside of a domain of finite area $E$ is arbitrarily close to horizontal and $|\nabla r|$ is almost equal to one, we conclude that the area of $E \cap\{r \leq t\}$ grows at most quadratically as $t \rightarrow \infty$. In fact, from (14) and (15) it follows that

$$
\int_{E \cap\{r \leq t\}} d A=\frac{C}{2} t^{2}+o(t),
$$

where $t^{-2} o(t) \rightarrow 0$ as $t \rightarrow \infty$. We now check that the constant $C$ must be an integer multiple of $2 \pi$. The locally finite minimal integral varifolds associated to the homothetically shrunk surfaces $\frac{1}{n} E$ converge as $n \rightarrow \infty$ to a locally finite minimal integral varifold with empty boundary which is contained in the $\left(x_{1}, x_{2}\right)$ plane. Since this limit varifold must is an integer multiple of the $\left(x_{1}, x_{2}\right)$-plane, $C$ must be an integer multiple of $2 \pi$.

In the case that the end $E$ "lies between catenoids", a similar analysis (see $[\mathbf{2 6}]$ for details) using the universal superharmonic function $\ln r-c\left(x_{3} \arctan \left(x_{3}\right)-\right.$ $\frac{1}{2} \ln \left(x_{3}^{2}+1\right)$ ), for some $c>0$, shows that $E \cap\{r \leq t\}$ has area growth $n \pi t^{2}$ for some $n \in \mathbb{N}$. This in turn implies that $E$ has area growth $n \pi R^{2}$ where $R=$ $\sqrt{x_{1}^{2}+x_{2}^{2}+x_{3}^{2}}$, in the sense that $\lim _{t \rightarrow \infty} \frac{\operatorname{Area}(M \cap\{R \leq t\})}{\pi t^{2}}=n$.

By the monotonicity formula for area (Theorem 4), every end representative of a minimal surface must have area growth at least equal to the area growth of a plane which is $\pi R^{2}$. Since we just checked that middle ends of a properly embedded minimal surface have quadratic area growth, then we can find representatives for middle ends which have exactly one end, which means they are never limit ends. This discussion gives the next Theorem and we refer the reader to [26] for further details.

Theorem 19 (Collin, Kusner, Meeks, Rosenberg [26]). Let $M \subset \mathbb{R}^{3}$ be a properly embedded minimal surface with more than one end and horizontal tangent 
plane at infinity. Then, any limit end of $M$ must be a top or bottom end of $M$. In particular, $M$ can have at most two limit ends, each middle end is simple and the number of ends of $M$ is countable. Furthermore, each middle end $E$ of $M$ has limiting quadratic area growth $m(E) \pi R^{2}$ as $R \rightarrow \infty$, where $m(E)$ is a positive integer. The parity of $m(E)$ is called the parity of the middle end $E$.

Collin, Kusner, Meeks and Collin [26] were also able to use universal superharmonic functions to control the geometry of properly embedded minimal surfaces with exactly two limit ends. Their proof of the following Theorem is motivated by the proof of a similar theorem by Callahan, Hoffman and Meeks [7] in the classical 1-periodic setting.

THEOREM 20. Let $M \subset \mathbb{R}^{3}$ be a properly embedded minimal surface with two limit ends and horizontal limit tangent plane at infinity. Then there exists a proper collection $\left\{P_{n} \mid n \in \mathbb{Z}\right\}$ of horizontal planes in $\mathbb{R}^{3}$ such that every plane intersects $M$ transversely in a finite number of simple closed curves. Furthermore, the closed slab $S_{n}$ bounded by $P_{n} \cup P_{n+1}$ intersects $M$ in a noncompact domain which represents the $n$-th end of $M$. In particular, by Theorem 12, $M$ is recurrent.

\section{Maximum principle at infinity for properly immersed minimal surfaces.}

In this Section we will present a sketch of the proof of a general maximum principle at infinity. As an important application of it, we will also show the existence of tubular neighborhoods for any properly embedded minimal surface with bounded Gaussian curvature, which in turn implies that the area growth of such a surface in balls is not more than cubical in the radial function $R$. The next statement generalizes the maximum principle at infinity described in Theorem 6 to the case of noncompact boundaries.

Theorem 21 (Maximum principle at infinity [79, 121]). Let $M_{1}, M_{2} \subset N^{3}$ be disjoint connected properly immersed minimal surfaces with possibly empty boundaries in a complete flat 3-manifold $N^{3}$.

i) If $\partial M_{1} \neq \varnothing$ or $\partial M_{2} \neq \varnothing$, then

$$
\operatorname{dist}\left(M_{1}, M_{2}\right)=\min \left\{\operatorname{dist}\left(\partial M_{1}, M_{2}\right), \operatorname{dist}\left(\partial M_{2}, M_{1}\right)\right\} .
$$

ii) If $\partial M_{1}=\partial M_{2}=\varnothing$, then $M_{1}$ and $M_{2}$ are flat.

Sketch OF THE PROOF. The only real difference between the statements of Theorem 6 and of the above Theorem is that the boundaries of the surfaces in Theorem 21 are allowed to be noncompact. This noncompactness property presents some serious technical difficulties that are not easy to overcome. The most difficult of these problems arises from the unknown conformal structure of a stable orientable complete minimal surface $\Sigma$ with boundary, which we now explain. By Theorem 9, when $\Sigma$ has compact boundary, then it has finite total curvature and so it is conformally a finitely punctured compact Riemann surface with compact boundary. In particular, when $\Sigma$ has compact boundary, then it is parabolic. On the other hand, at the end of Section 3 we discussed an example of a complete orientable stable minimal surface with noncompact boundary which was not parabolic. The fact that a stable minimal surface need not be parabolic makes the proof of the general maximum principle at infinity quite delicate. We now give the sketch of the proof and will eventually indicate how one circumvents this problem with 
the conformal structure. Note that by lifting the surfaces to the universal cover $\mathbb{R}^{3}$ of $N^{3}$, we may assume $N^{3}=\mathbb{R}^{3}$.

Assume that Theorem 21 fails for some properly immersed minimal surfaces $M_{1}, M_{2}$. The first step in the proof of Theorem 21 is to reduce to the case where $M_{1}, M_{2}$ are properly embedded and stable. To do this one uses $M_{1} \cup M_{2}$ together with small $\varepsilon$-neighborhoods of their boundary curves as barriers to obtain stable disjoint minimal surfaces $M_{1}^{\prime}, M_{2}^{\prime}$, where $\partial M_{i}^{\prime}$ lies in the boundary of the $\varepsilon$ neighborhood of $\partial M_{i}$ for $i=1,2$. This barrier argument is similar to one described in Subsection 4.2. Furthermore, the new stable surfaces are also constructed to be at least as close to each other as the previous ones and so by choosing $\varepsilon$-sufficiently small, $M_{1}^{\prime}, M_{2}^{\prime}$ are new counterexamples to the Theorem. So assume from now on that $M_{1}, M_{2}$ are properly embedded and stable.

Next we show how to complete the proof in the case that $\partial M_{1}$ and $\partial M_{2}$ are both compact (therefore proving Theorem 6). In this case, Theorem 9 implies that $M_{1}, M_{2}$ are two properly embedded connected minimal surfaces in $\mathbb{R}^{3}$ which have compact boundary and finite total curvature. Since the Theorem fails for $M_{1}, M_{2}$, then, after a fixed translation of $M_{1}$, we may assume that the distance from $M_{1}$ to $M_{2}$ is zero but the distances from $\partial M_{1}$ to $M_{2}$ and from $\partial M_{2}$ to $M_{1}$ are both positive. The only way that this can happen is that an end $E_{1}$ of $M_{1}$ has distance zero to some end $E_{2}$ of $M_{2}$. Since the ends of embedded finite total curvature surfaces can be taken to be graphs which are planar (zero logarithmic growth) or of catenoid-type (bounded logarithmic growth), we may assume after a rigid motion of $\mathbb{R}^{3}$ that $E_{1}$ and $E_{2}$ are nonnegative graphs over an annular domain $A=\left\{\left(x_{1}, x_{2}\right) \mid x_{1}^{2}+x_{2}^{2} \geq R_{0}^{2}\right\}$ with some fixed nonnegative logarithmic growth. In this case the estimate in formula (9) implies $E_{1}$ and $E_{2}$ are both asymptotic to the end of a common plane or to the end of a fixed catenoid. In particular, $E_{1}$ and $E_{2}$ are asymptotic to each other.

Suppose that the $E_{1}$ lies below $E_{2}$. After a small upward translation of $E_{1}$, we obtain a surface $E_{1}^{\prime}$ whose end lies above the end of $E_{2}$ and whose boundary lies below the boundary of $E_{2}$. An application of the usual maximum principle implies that $\Gamma=E_{1}^{\prime} \cap E_{2}$ is a simple closed homotopically nontrivial curve on both surfaces. Let $E_{1}^{\prime}(\Gamma) \subset E_{1}^{\prime}$ and $E_{2}(\Gamma) \subset E_{2}$ be the annular ends of these surfaces with common boundary $\Gamma$. Note that the third component of the conormal to $E_{1}^{\prime}(\Gamma)$ along $\Gamma$ is pointwise greater in norm than the corresponding third component of the conormal to $E_{2}(\Gamma)$ along $\Gamma$, since $E_{1}^{\prime}(\Gamma)$ lies above $E_{2}(\Gamma)$ along $\Gamma$. It follows that the vertical component of the flux of $E_{1}^{\prime}$ is greater in norm than the vertical component of the flux of $E_{2}$. But these vertical fluxes of $E_{1}^{\prime}$ and $E_{2}$ depend only on their logarithmic growths, which are equal. This contradiction completes the proof of the case where $\partial M_{1}$ and $\partial M_{2}$ are both compact.

We now continue with our proof in the case $\partial M_{1}$ or $\partial M_{2}$ is not compact. By our previous arguments, $M_{1}$ and $M_{2}$ can be supposed stable and properly embedded and, after a possible translation, have $\operatorname{dist}\left(M_{1}, M_{2}\right)=0$ but with both $\operatorname{dist}\left(\partial M_{1}, M_{2}\right)$ and $\operatorname{dist}\left(\partial M_{2}, M_{1}\right)$ being positive. By curvature estimates for stable minimal surfaces (Theorem 15), after removing small regular neighborhoods of their boundaries, the Gaussian curvature of each of the surfaces is bounded. Since $M_{1}$ has bounded Gaussian curvature, there exists $\varepsilon>0$ such that the natural exponential map from the normal bundle $N\left(M_{1}\right)$ of $M_{1}$ into $\mathbb{R}^{3}$ restricts as a submersion to the subbundle $N_{\varepsilon}\left(M_{1}\right)=\left\{\xi \in N\left(M_{1}\right) \mid\|\xi\|<\varepsilon\right\}$. Note that $M_{1}$ can be seen as the zero section of $N_{\varepsilon}\left(M_{1}\right)$. Since some points of the interior of $M_{2}$ approach points of 
the interior of $M_{1}$, then after pulling-back portions of $M_{2}$ to $N_{\varepsilon}\left(M_{1}\right)$ we can find proper domains $\Omega_{1} \subset M_{1}, \Omega_{2} \subset M_{2} \cap N_{\varepsilon}\left(M_{1}\right)$ such that $\Omega_{2}$ is a normal graph over $\Omega_{1}$ with boundary values $\varepsilon$ and $\operatorname{dist}\left(\Omega_{1}, \Omega_{2}\right)=0$.

If $\Omega_{1}$ is parabolic, then using the minimal surface equation for such a normal graph for $\varepsilon$ small, one can produce a positive proper superharmonic function on $\Omega_{1}$ which does not have its minimum on the boundary of $\Omega_{1}$, which contradicts the maximum principle. Hence, $\Omega_{1}$ is not parabolic. In fact, after removing any sufficiently small fixed size regular neighborhood of $\partial \Omega_{1}$ from $\Omega_{1}$, this argument shows that we still obtain a surface which cannot be parabolic. This problem motivates the following definition.

Definition 20. A Riemannian surface $M$ with boundary is $\varepsilon$-parabolic if for any $\varepsilon>0, M(\varepsilon)=\{p \in M \mid \operatorname{dist}(p, \partial M) \geq \varepsilon\}$ is a parabolic surface.

Hence, to complete the proof of Theorem 21 it is sufficient to prove that $\Omega_{1}$ is $\varepsilon$-parabolic. While it must certainly be the case that $\Omega_{1}$ is $\varepsilon$-parabolic, the proof of this property is not what is shown. Rather one proves that between the zero section $\Omega_{1}$ and the graph $\Omega_{2}$, there are normal minimal graphs $\Delta(t)$ over $\Omega_{1}$ for every $0<t<\varepsilon$. One of these graphs $\Delta\left(t_{0}\right)$ is a limit of the other graphs and such that the limit induces a positive Jacobi function $u$ on $\Delta\left(t_{0}\right)$ which is bounded away from zero in a fixed sized neighborhood of its boundary. The arguments of Fischer-Colbrie in [34] in her proof of Theorem 9 now imply that if $g$ is the metric for $\Delta\left(t_{0}\right)$, then $u \cdot g$ is a new metric which is complete and has nonpositive Gaussian curvature. One then proves that such surfaces are $\varepsilon$-parabolic, which easily implies that $\Delta\left(t_{0}\right)$ is $\varepsilon$-parabolic in the original metric. Once one obtains such a $\Delta\left(t_{0}\right)$, then using the previous arguments one rather easily arrives at a contradiction. This completes our sketch of the proof to Theorem 21 .

We now come to a beautiful and deep application of the general maximum principle at infinity. The next Corollary appears in [79] and a slightly weaker variant of it in [121].

Corollary 2. Suppose $M \subset \mathbb{R}^{3}$ is a properly embedded minimal surface with absolute bounded Gaussian curvature at most 1. Let $N_{1}(M)$ be the subbundle of the normal bundle of $M$ given by the vectors of length strictly less that 1 , and let $\exp : N_{1}(M) \rightarrow \mathbb{R}^{3}$ be the corresponding exponential map. Then exp is a smooth embedding. In particular, $M$ has a open embedded tubular neighborhood of radius 1.

Instead of giving a proof of Corollary 2, we will only check that a minimal surface $M$ under the hypotheses in Corollary 2 has some tubular neighborhood. Consider $X=\exp ^{-1}(M) \subset N_{1}(M)$ and note that $X$ contains the zero section $M$ of $N_{1}(M)$. Suppose $X$ contains a component $\Delta$ which is different from the zero section. In this case, the distance from $\partial \Delta$ to $M$ is equal to 1 , but the distance from $\Delta$ to $M$ is strictly less that 1 . Since exp: $N_{1}(M) \rightarrow \mathbb{R}^{3}$ is a submersion, under the pulled-back metric we can view $N_{1}(M)$ as a flat three-manifold. Inside this flat three-manifold, $\Delta$ and $M$ contradict the maximum principle at infinity, or rather the proof of Theorem 21 holds in this setting and so gives the desired contradiction. We conclude that $X=M$. But then a simple application of the triangle inequality implies the restricted map exp $\left.\right|_{N_{\frac{1}{2}}(M)}$ is injective and so $M$ has a tubular neighborhood of radius $\frac{1}{2}$. A special barrier argument is used to obtain the optimal maximal radius 1 tubular neighborhood given in the statement of the Corollary. 


\section{Flux conjectures and some properties of harmonic functions in parabolic Riemannian manifolds.}

Algebraic-analytic invariants associated to a properly embedded minimal surface $M \subset \mathbb{R}^{3}$ are a way of not only distinguishing $M$ from other such examples but also can be used as theoretical tools for acquiring deeper information on the surface. One of the most natural of these invariants is the flux of $M$ associated to a Killing vector field $V$ of $\mathbb{R}^{3}$. Given such a $V$ and its restriction $V_{M}$ to $M$, let $V_{M}^{T}$ denote the tangent vector field on $M$ which is the tangential part of $V_{M}$; note that the normal projection $V_{M}^{N}=V_{M}-V_{M}^{T}$ is a Jacobi vector field (i.e. the inner product of $V_{M}$ with the Gauss map $N$ of $M$ is a Jacobi function). Given two oriented homologous cycles $\gamma_{1}, \gamma_{2} \subset M$, the Divergence Theorem implies that the flux $\int_{\gamma_{1}}\left\langle V_{M}^{T}, \eta\right\rangle d s$ of $V_{M}^{T}$ across $\gamma_{1}$ is equal to its flux across $\gamma_{2}$ (here $\eta$ stands for the unit conormal vector to $M$ along the integration curve). When the Killing vector field $V$ is one of the parallel vector fields $e_{1}, e_{2}, e_{3}$ induced by the standard coordinate functions in $\mathbb{R}^{3}$, then for $V=e_{i}$ is $V_{M}^{T}=\nabla x_{i}$ where $\nabla x_{i}$ is the intrinsic gradient of the coordinate function $x_{i}$ on $M$. Thus, for each homology class $\gamma \in H_{1}(M, \mathbb{Z})$, one obtains the flux vector $F(\gamma)$ defined in equation (8).

There is a well-known conjecture of Meeks concerning precisely when the so called flux map $F: H_{1}(M, \mathbb{Z}) \rightarrow \mathbb{R}^{3}$ is the zero map, or equivalently, it describes precisely which surfaces $M$ have zero flux.

Conjecture 1 (Flux Conjecture (I)). If $M \subset \mathbb{R}^{3}$ is a properly embedded minimal surface with zero flux, then $M$ is a plane or a helicoid.

Because of the characterization by Meeks and Rosenberg (Theorem 30 below) of the plane and the helicoid as the unique properly embedded simply connected minimal surfaces in $\mathbb{R}^{3}$, Conjecture 1 can be equivalently stated as follows.

CONJECTURe 2 (Flux Conjecture (II)). If $M \subset \mathbb{R}^{3}$ is a properly embedded nonsimply connected minimal surface, then $M$ has nonzero flux.

An isometric minimal immersion $X:\left(M, d s^{2}\right) \rightarrow \mathbb{R}^{3}$ is rigid if given another isometric minimal immersion $Y:\left(M, d s^{2}\right) \rightarrow \mathbb{R}^{3}$, there exists a rigid motion $R: \mathbb{R}^{3} \rightarrow$ $\mathbb{R}^{3}$ such that $R \circ X=Y$. On the other hand, if $X$ has Weierstrass data $(g, d h)$, then for each $\theta \in[0,2 \pi)$ the pair $\left(g, e^{i \theta} d h\right)$ defines via Theorem 1 a possibly multivalued ${ }^{8}$ minimal surface $X_{\theta}$, which is called an associate surface of $X$. The associate surface for $\theta=\pi / 2$ is the conjugate surface. Equation (6) implies that two associate minimal surfaces are locally isometric. The converse is true by Calabi's Rigidity Theorem [5], which asserts that if $X, Y: M \rightarrow \mathbb{R}^{3}$ are isometric minimal immersions and they are locally isometric, then $Y$ is congruent to an associate surface of $X$.

Lemma 2. Let $X: M \rightarrow \mathbb{R}^{3}$ be a isometric minimal immersion. Then, the following statements are equivalent:

1. $X$ is rigid.

2. All associate surfaces to $X$ are multivalued, except $X$ and $X_{\pi}$.

3. There exists a cycle $\gamma \in H_{1}(M, \mathbb{Z})$ such that the flux $F(\gamma)$ is not zero.

Proof. If $X$ is rigid and $\theta \in[0,2 \pi)$, then the pulled-back metrics by $X, X_{\theta}$ coincide, hence if $X_{\theta}$ is univalent then $R \circ X_{\theta}=X$ for certain rigid motion $R$. Since

\footnotetext{
${ }^{8}$ In the sense that $X_{\theta}$ may have real periods although $X$ solves the period problem.
} 
$X, X_{\theta}$ share the Gauss map, the linear part of $R$ must be the identity $I$ or $-I$ and so, $\theta=0$ or $\pi$, which proves $1 \Rightarrow 2$. The implication $2 \Rightarrow 3$ follows easily from the fact that the flux vector of $X$ on a given cycle $[\gamma] \in H_{1}(M, \mathbb{Z})$ is a period for its conjugate surface. To see $3 \Rightarrow 1$, take an isometric minimal immersion $Y: M \rightarrow \mathbb{R}^{3}$. By Calabi's Rigidity Theorem, there exists a rigid motion $R: \mathbb{R}^{3} \rightarrow \mathbb{R}^{3}$ and an associate surface $X_{\theta}$ such that $R \circ X_{\theta}=Y$. In particular, $X_{\theta}$ is well-defined on $M$. By hypothesis, there exists $\gamma \in H_{1}(M, \mathbb{Z})$ such that the $F(\gamma) \neq 0$. Since the period of $X_{\theta}$ along $\gamma$ equals $\sin \theta \cdot F(\gamma)$, it follows that $\theta=0$ or $\pi$, thus $X$ and $Y$ are congruent.

Lemma 2 insures that the helicoid is not minimally rigid. Meeks and Rosenberg $[\mathbf{8 1}]$ proved that every nonflat doubly periodic minimal surface $M \subset \mathbb{R}^{3}$ with finite topology in the quotient has a cycle with nonzero flux. Meeks [71] extended this property to all triply periodic minimal surfaces and to singly periodic minimal surfaces with finite topology in the quotient and planar or Scherk-type ends. By Lemma 2 all these surfaces are rigid, and the helicoid shows that we cannot expect to extend these results to singly periodic minimal surfaces with helicoidal type ends. Pérez [104] showed that no other nonrigid example can exist in this last family of surfaces. All these results together imply that if a $M$ is a nonflat properly embedded periodic minimal surface in $\mathbb{R}^{3}$ with finite topology in the quotient, then $M$ is rigid or it is the helicoid.

An easy consequence of the definition of rigidity is that in a rigid minimal surface, every intrinsic isometry extends to an ambient isometry of $\mathbb{R}^{3}$ (this property is sometimes referred to as weak rigidity). Since intrinsic isometries of the helicoid and the plane extend to ambient isometries, the validity of the equivalent Conjectures 1 or 2 would imply the following conjecture by Meeks:

Conjecture 3 (Isometry Conjecture). If $M \subset \mathbb{R}^{3}$ is a properly embedded minimal surface, then every intrinsic isometry of $M$ extends to an ambient isometry.

By the above arguments, Conjecture 3 holds when $M$ is periodic and has finite topology in the quotient. In 1990, Choi, Meeks and White [12] proved that the stronger Conjecture 2 holds when $M$ has more than one end and very recently, Meeks and Rosenberg (Theorem 30) extended the validity of Conjecture 2 to the case of $M$ having one end and finite genus. In summary, Conjectures 1, 2 and 3 can only fail if the surface $M$ has exactly one end and infinite genus. By Lemma 2, one way to prove Conjecture 1,2 and 3 would be to show that when $M$ has one end and infinite genus, then there exists a plane in $\mathbb{R}^{3}$ that intersects $M$ in a set that contains a simple closed curve.

Another important conjecture related to flux, which is also closely related to classification questions, is the following one due to Meeks, Pérez and Ros.

Conjecture 4 (Flux Conjecture (III)). If $M \subset \mathbb{R}^{3}$ is a properly embedded minimal surface and the rank of the flux map is 1 , then $M$ is the catenoid, one of the Riemann minimal examples or one of the Scherk doubly-periodic examples defined in Subsection 2.5.

For a minimal surface $M \subset \mathbb{R}^{3}$ whose flux map has rank 1 we have at our disposal a powerful tool: the López-Ros deformation introduced in Subsection 2.2. This tool together with the maximum principle for minimal surfaces were crucial when proving certain uniqueness and nonexistence results, see for instance [63, 
104, 105]. The interested reader can find an exposition of both this tool and some of its applications in Pérez and Ros [107].

By Collin's Theorem 3 and the López-Ros [63] characterization of the catenoid, Conjecture 4 holds when $M$ has finite topology and more than on end (the only possible surface in this case is the catenoid). On the other hand, Theorems 31 and 32 below imply that any nonsimply connected properly embedded minimal surface $M \subset \mathbb{R}^{3}$ of genus zero has flux map of rank 1, and so a positive solution to Conjecture 4 would complete the classification of genus zero minimal surfaces in $\mathbb{R}^{3}$.

We now describe a recently discovered flux invariant associated to each coordinate function of a properly immersed minimal surface $M$ in $\mathbb{R}^{3}$. Suppose $x: M \rightarrow \mathbb{R}$ is a coordinate function and $t \in \mathbb{R}$. Consider the scalar flux (possibly infinite) $\int_{x^{-1}(t)}|\nabla x| d s$ of the intrinsic gradient $\nabla x$ across the level set $x^{-1}(t)$, which is oriented almost everywhere by the normal vector $\left.(\nabla x)\right|_{x^{-1}(t)}$. If $t_{1} \leq t_{2}$ are real numbers, then Theorem 12 implies that $M\left[t_{1}, t_{2}\right]=x^{-1}\left[t_{1}, t_{2}\right]$ is a parabolic manifold. Thus, by the following Lemma, the scalar flux of $\nabla x$ across a level set is independent of the level set; we will call this number the flux of $\nabla x$.

Lemma 3 (Flux Lemma, Meeks [67]). Suppose $M$ is a parabolic Riemannian manifold, $h: M \rightarrow[0,1]$ is a nonconstant harmonic function and $\partial M=$ $h^{-1}(\{0,1\})$. Then, the flux of $\nabla h$ across $h^{-1}(0)$ equals the flux of $\nabla h$ across $h^{-1}(1)$.

The above Lemma is a consequence of Green's Theorem and the definition of a parabolic manifold. Meeks has made the following related Conjecture.

Conjecture 5 (Finite Geometric Flux Conjecture). Let $M$ be a parabolic Riemannian manifold and $h: M \rightarrow[0,1]$ a nonconstant harmonic function with $\partial M=h^{-1}(\{0,1\})$. If $\nabla h$ has finite flux across $h^{-1}(0)$, then almost all integral curves of $\nabla h$ begin at $h^{-1}(0)$ and end at $h^{-1}(1)$.

Meeks and Wolf [87] have been able to prove Conjecture 5 in the case of dimension two. We finish this Section with a related problem to Conjecture 5. This problem constitutes one of the deepest conjectures in classical minimal surface theory and it is due to Meeks and Rosenberg.

Conjecture 6 (Geometric Flux Conjecture). Suppose $M \subset \mathbb{R}^{3}$ is a properly embedded minimal surface. Then, except for a countable subset, the integral curves of a given coordinate function begin at $-\infty$ and end at $+\infty$.

By the results contained in Theorem 3, Theorem 30, Theorem 31 and Theorem 32, the Geometric Flux Conjecture holds when $M$ has finite genus.

\section{The Colding-Minicozzi curvature estimates, compactness and regularity of limit laminations and applications.}

The famous open spherical space-form problem asks if any free action of a finite group $\Gamma$ of diffeomorphisms of the sphere $\mathbb{S}^{3}$ is conjugate to an action by isometries in $O(4)$. According to Pitts and Rubinstein [108], the failure of this question to be solved in the affirmative would create by minimax methods a sequence of embedded minimal unknotted tori $M_{n}$ in some $\Gamma$-invariant metric on $\mathbb{S}^{3}$ with the index of stability of $M_{n}$ at least $n$. This approach motivated the following open question by Pitts and Rubenstein. 
QUESTION 2. Does there exist a bound for the stability index of all closed embedded minimal surfaces with fixed genus in a given closed 3 -manifold $N$ with respect to some large class of Riemannian metrics?

Arguments in Choi and Schoen [11] imply that if $N$ is a closed Riemannian 3 -manifold with a generic metric, then there exists a bound for the stability index of all closed embedded minimal surfaces in $N$ with fixed genus and a fixed area bound. Thus, the above question by Pitts and Rubinstein leads to the study of sequences of closed embedded minimal surfaces with fixed genus in such a $N^{3}$ but without area (or density) bounds. Viewing a sequence of embedded minimal surfaces in $N$ as a sequence of Radon measures on $N$, compactness results from Geometric Measure Theory imply that a subsequence of the measures (renormalized to have fixed mass) converges weakly to another Radon measure. The problem of understanding the geometric structure of this limit starts with the analysis of the local structure in a fixed ball, which motivates the central problem tackled by Colding and Minicozzi in a recent series of papers: the local structure of a limit of compact embedded minimal surfaces $M_{n}$ with fixed genus and no area bound in a ball $B \subset \mathbb{R}^{3}$, with boundaries $\partial M_{n} \subset \partial B$. The most important case of their structure Theorem is when the $M_{n}$ are disks and their Gaussian curvature blows up near the origin.

The basic example in this setting is a sequence of rescaled helicoids $M_{n}=a_{n} H$ where $H$ is a fixed vertical helicoid with axis the $x_{3}$-axis and $a_{n} \in \mathbb{R}^{+}, a_{n} \rightarrow 0$. The curvature of the sequence $\left\{M_{n}\right\}_{n}$ blows up along the $x_{3}$-axis and the $M_{n}$ converge away from the axis to a foliation $\mathcal{L}$ of $\mathbb{R}^{3}$ by horizontal planes. The $x_{3}$-axis is the singular set of convergence $S(\mathcal{L})$ of $M_{n}$ to $\mathcal{L}$, but each leaf of $\mathcal{L}$ extends smoothly across its intersection with $S(\mathcal{L})$ (i.e. $S(\mathcal{L})$ consists of removable singularities of $\mathcal{L})$. With this model in mind, the statement of the so called Limit Lamination Theorem (Theorem 0.1 of [18]) can be easily understood. Given $p \in \mathbb{R}^{3}$ and $R>0$, we denote by $B(p, R)=\left\{x \in \mathbb{R}^{3} \mid\|x-p\|<R\right\}, B(R)=B(0, R)$ and $K_{M}$ the Gaussian curvature function of a surface $M$.

Theorem 22 (Colding, Minicozzi [18]). Let $M_{n} \subset B\left(R_{n}\right)$ be a sequence of embedded minimal disks with $\partial M_{n} \subset \partial B\left(R_{n}\right)$ and $R_{n} \rightarrow \infty$. If $\sup \left|K_{M_{n} \cap B(1)}\right| \rightarrow$ $\infty$, then there exists a subsequence of the $M_{n}$ (denoted in the same way) and a Lipschitz curve $S: \mathbb{R} \rightarrow \mathbb{R}^{3}$ such that up to a rotation of $\mathbb{R}^{3}$,

1. $x_{3}(S(t))=t$ for all $t \in \mathbb{R}$.

2. For each compact domain $C \subset \mathbb{R}^{3}-S$ and $n$ large enough, $M_{n} \cap C$ consists of two multivalued graphs over a subdomain of $\left\{x_{3}=0\right\}$ with $\left\{M_{n} \cap C\right\}_{n}$ converging to $C \cap \mathcal{L}$ as $n \rightarrow \infty$ in the $C^{\alpha}$-topology for any $\alpha \in(0,1)$, where $\mathcal{L}=\left\{x_{3}=t\right\}_{t \in \mathbb{R}}$ is the foliation of $\mathbb{R}^{3}$ by horizontal planes.

3. $\sup \left|K_{M_{n} \cap B(S(t), r)}\right| \rightarrow \infty$ as $n \rightarrow \infty$, for any $t \in \mathbb{R}$ and $r>0$.

Theorem 22 has two main ingredients in its proof, which we explain very roughly. The first ingredient is that the embedded minimal disk $M_{n}$ with large curvature at some interior point can be divided into building blocks, each one being a multivalued graph $u_{n}(\rho, \theta)$ defined on an annulus ${ }^{9}$, and that these basic pieces fit together properly. In particular, they prove that the number of sheets of $u_{n}(\rho, \theta)$

\footnotetext{
${ }^{9}$ In polar coordinates $(\rho, \theta)$ with $\rho>0$ and $\theta \in \mathbb{R}$, a $N$-valued graph on an annulus of inner radius $r$ and outer radius $R$, is a single valued graph of a function $u(\rho, \theta)$ defined over $\{(\rho, \theta)|r \leq \rho \leq R,| \theta \mid \leq N \pi\}, N$ being a positive integer. The separation between consecutive sheets is $w(\rho, \theta)=u(\rho, \theta+2 \pi)-u(\rho, \theta) \in \mathbb{R}$.
} 
rapidly grows as the curvature blows up and at the same time the sheets do not accumulate in a halfspace. This is obtained by means of sublinear and logarithmic bounds for the separation ${ }^{9} w_{n}(\rho, \theta)$ as a function of $\rho \rightarrow \infty$. Another consequence of these bounds is that by allowing the inner radius ${ }^{9}$ of the annulus where the multigraph is defined to go to zero, the sheets of this multigraph collapse (i.e. $\left|w_{n}(\rho, \theta)\right| \rightarrow 0$ as $n \rightarrow \infty$ for $\rho, \theta$ fixed), thus a subsequence of the $u_{n}$ converges to a smooth minimal graph through $\rho=0$. The fact that the $R_{n}$ go to $\infty$ then implies this limit graph is entire and, by Bernstein Theorem [1] , it is a plane.

The second main ingredient in the proof of Theorem 22 is the so called onesided curvature estimate, a scale invariant bound for the Gaussian curvature of embedded minimal disks in a halfspace.

Theorem 23 (Colding, Minicozzi [18]). There exists $\varepsilon>0$ such that the following holds. Given $r>0$ and an embedded minimal disk $M \subset B(2 r) \cap\left\{x_{3}>0\right\}$ with $\partial M \subset \partial B(2 r)$, then for any component $M^{\prime}$ of $M \cap B(r)$ which intersects $B(\varepsilon r)$,

$$
\sup _{M^{\prime}}\left|K_{M}\right| \leq r^{-2} .
$$

The hypothesis on $M$ to be simply-connected in Theorem 23 is necessary, as the catenoid demonstrates. Theorem 23 basically says that if an embedded minimal disk is close enough to (and lies at one side of) a plane, then reasonably large components of it are graphs over this plane. This result is needed in the proof of Theorem 22 in the following manner: once it has been proven that an embedded minimal disk $M$ contains a 2 -valued graph $\widetilde{M}$, then $\widetilde{M}$ plays the role of the plane in the onesided curvature estimate which implies that reasonably large pieces of $M$ consist of multivalued graphs away from a cone with axis "orthogonal" to the 2-valued graph. The proofs of Theorems 22 and 23 are long and delicate. References $[\mathbf{2 4}, \mathbf{1 3}, \mathbf{1 4}]$ by Colding and Minicozzi are reading guides for the complete proofs of these results, which go through various papers $[\mathbf{1 5}, \mathbf{1 6}, \mathbf{1 8}]$; see also the forthcoming survey by the authors in $[\mathbf{7 3}]$.

Theorems 22 and 23 have been applied to obtain a number of results. For instance, with these results in hand Meeks and Rosenberg [80] proved that the helicoid and the plane are the unique simply connected properly embedded minimal surfaces in $\mathbb{R}^{3}$ (see Theorem 30) and Meeks, Pérez and Ros showed that no properly embedded minimal surfaces with finite genus and one limit end can exist (Theorem 31). We will discuss other applications of Colding-Minicozzi results in Theorems 29 and 32 .

Definition 21. A lamination of an open subset $O \subset \mathbb{R}^{3}$ is the union of a collection of pairwise disjoint connected complete injectively immersed surfaces. More precisely, it is a pair $(\mathcal{L}, \mathcal{A})$ satisfying

1. $\mathcal{L}$ is a closed subset of $O$;

2. $\mathcal{A}=\left\{\varphi_{\alpha}: \mathbb{D} \times(0,1) \rightarrow U_{\alpha}\right\}_{\alpha}$ is a collection of coordinate charts of $\mathbb{R}^{3}$ (here $\mathbb{D}$ is the open unit disk, $(0,1)$ the open unit interval and $U_{\alpha}$ an open subset of $O)$;

3. For each $\alpha$, there exists a closed subset $C_{\alpha}$ of $(0,1)$ such that $\varphi_{\alpha}^{-1}\left(U_{\alpha} \cap \mathcal{L}\right)=$ $\mathbb{D} \times C_{\alpha}$.

We will simply denote laminations as $\mathcal{L}$, omitting the charts $\varphi_{\alpha}$ in $\mathcal{A}$. A lamination $\mathcal{L}$ is said to be a foliation of $O$ if $\mathcal{L}=O$. Every lamination $\mathcal{L}$ naturally decomposes into a union of disjoint surfaces, called the leaves of $\mathcal{L}$. As usual, the regularity 
of $\mathcal{L}$ requires the corresponding regularity on the change of coordinate charts. A lamination is minimal if all its leaves are minimal surfaces.

Each leaf $L$ of a minimal lamination $\mathcal{L}$ is smooth, and if $C \subset L$ is a compact subset of a limit leaf $L \in \mathcal{L}$, then the leaves of $\mathcal{L}$ converge smoothly to $L$ over $C$ (i.e. they converge uniformly in the $C^{k}$-topology on $C$ for any $k$ ).

Theorem 22 does not hold if we exchange the hypothesis that the radii $R_{n}$ of the balls go to $\infty$ by $R_{n}$ equals a constant, as demonstrated by a counterexample in [13]. Colding and Minicozzi construct a sequence of embedded minimal disks $M_{n} \subset B(1)$ with $\partial M_{n} \subset \partial B(1)$ all passing through the origin, and with Gaussian curvature blowing up only at the origin. This sequence produces a limit lamination of $B(1)-\{(0,0,0)\}$ with an isolated singularity at the origin. The limit lamination consists of three leaves, one of them being the flat horizontal punctured disk (which extends through the origin) and the other two being nonproper multigraphs with this disk as limit set. In particular, both smoothness and properness of leaves of the limit lamination fail for this local example.

Theorem 22 deals with limits of sequences of disks and it is also useful when studying more general situations, as for instance, uniformly locally simply connected sequences of minimal surfaces, a notion which we now define.

DEFINITION 22. Suppose $\left\{M_{n}\right\}_{n}$ is a sequence of properly embedded nonsimply connected minimal surfaces in $\mathbb{R}^{3}$. Given $p \in \mathbb{R}^{3}$ and $n \in N$, we let $r_{n}(p)>0$ be the largest radius of an open ball $B$ centered at $p$ such that $B$ intersects $M_{n}$ in simply connected components. If for any $p \in \mathbb{R}^{3}$ the sequence $\left\{r_{n}(p)\right\}_{n}$ is bounded away from zero, we say that $\left\{M_{n}\right\}_{n}$ is locally simply connected. If for all $p \in \mathbb{R}^{3}$, the radius $r_{n}(p)$ is bounded from below by a positive constant for all $n$ large, we will say that $\left\{M_{n}\right\}_{n}$ is uniformly locally simply connected (ULSC).

We have exploited the technique of blowing-up a sequence of immersed minimal surfaces on the scale curvature (see for instance the proof of Theorem 15 where we carry this out explicitly). When the surfaces in the sequence are properly embedded in $\mathbb{R}^{3}$, this blowing-up process produces a limit which is a properly embedded, nonflat minimal surface with bounded Gaussian curvature, whose genus and rank of homology groups are bounded by the ones for the $M_{n}$. For example, if each $M_{n}$ is a planar domain, then the same holds for the limit.

For useful applications of the concept of ULSC sequence, it is essential to consider sequences of properly embedded minimal surfaces which a priori may not satisfy the ULSC condition, and the modify them to produce a new sequence which satisfies that condition. We accomplish this by considering a blow-up argument on a geometric scale which, in general, is different from blowing-up on the scale of curvature. We call this procedure blowing-up by the scale of topology. This scale was defined and used in $[\mathbf{7 4}, \mathbf{7 5}]$ to prove that any properly embedded minimal surface of finite genus has bounded curvature and is recurrent for Brownian motion. We now explain the elements of this new scale.

Suppose $\left\{M_{n}\right\}_{n}$ is a sequence of nonsimply connected, properly embedded minimal surfaces which is not ULSC. Note that the Gaussian curvature of the collection $M_{n}$ is not uniformly bounded, and so, one could blow-up these surfaces on the scale of curvature to obtain a properly embedded nonflat minimal surface which may or may not be simply connected. Also note that, after choosing a subsequence, there exists points $p_{n} \in \mathbb{R}^{3}$ such that $r_{n}\left(p_{n}\right) \rightarrow 0$ as $n \rightarrow \infty$, where $r_{n}$ is the function appearing in Definition 22. Let $\widetilde{p}_{n}$ be a point in $B\left(p_{n}, 1\right)$ where the function 
$x \mapsto d\left(x, \partial B\left(p_{n}, r_{n}\left(p_{n}\right)\right)\right) \cdot r_{n}(x)$ attains its maximum. Then the translated and rescaled surfaces $\widetilde{M}_{n}=\frac{1}{r_{n}\left(\widetilde{p}_{n}\right)}\left(M_{n}-\widetilde{p}_{n}\right)$ intersect for all $n$ the closed ball centered at the origin with radius 1 in at least one component which is not simply connected, and for $n$ large they intersect any ball of radius less that 1 in simply connected components, see [74] for details.

For the sake of clarity, we now illustrate this blow-up procedure on certain sequences of Riemann minimal examples, defined in Subsection 2.5. Each of these surfaces is foliated by circles and straight lines in horizontal planes, with a vertical plane of symmetry that can be assumed to be the $\left(x_{1}, x_{3}\right)$-plane. After a translation and a homothety, we assume that these surfaces are normalized so that the corresponding function $r$ defined above attains its minimum value of 1 at the origin. Under this normalization, any sequence of Riemann minimal examples is ULSC. The flux of each Riemann minimal example along a compact horizontal section has horizontal and vertical components which are not zero, and the ratio $V$ of the norm of its horizontal component over the vertical one parametrizes the 1-parameter family of these surfaces, with $V \in(0, \infty)$. When $V \rightarrow 0$, the Riemann minimal examples converge smoothly to the vertical catenoid centered at the origin with waist circle of radius 1 . When $V \rightarrow \infty$, the Riemann minimal examples converge smoothly to a foliation of $\mathbb{R}^{3}$ by horizontal planes away from the two vertical lines passing through $(0,-1,0),(0,1,0)$. In a neighborhood of any compact arc on these lines, the limiting Riemann examples are arbitrarily closed to a high sheeted vertical helicoid with axis along the line. Since the $\left(x_{1}, x_{3}\right)$-plane is a plane of symmetry of the approximated surfaces, these limit helicoids are oppositely handed. Outside the vertical cylinders containing the highly sheeted helicoids, the Riemann minimal examples consist of two multivalued almost flat graphs, which outside of a bigger cylinder containing both helicoids, are univalent graphs representing each of the ends of the Riemann minimal examples. This picture describes one particular case of what we call a parking garage structure for a surface. Roughly speaking, a parking garage structure with $n$ columns is a smooth embedded surface in a horizontal slab $S \subset \mathbb{R}^{3}$ that can be decomposed into 2 disjoint almost flat horizontal multigraphs over the exterior of $n$ disjoint disks in the $\left(x_{1}, x_{2}\right)$-plane, together $n$ topological strips each one contained in one of the solid cylinders (these are the columns), such that each strip lies in a small regular neighborhood of the intersection of a vertical helicoid with $S$. One can associate to each column a + or - sign depending on the handedness of the corresponding helicoid. Note that a vertical helicoid is the basic example of a parking garage with 1 column, and the Riemann surfaces with $V \rightarrow \infty$ have the structure of a parking garage with two columns oppositely handed in any fixed size horizontal slab. Other parking garage structures with varying numbers of columns and associated signs can be found for other minimal surfaces, see Traizet and Weber [124].

There are interesting cases where ULSC guarantees the convergence of a sequence of minimal surfaces in $\mathbb{R}^{3}$ to a parking garage structure. Typically one proves that the sequence converges (up to a subsequence and a rotation) to a foliation of $\mathbb{R}^{3}$ by horizontal planes with singular set of convergence consisting of a locally finite set of Lipschitz curves parametrized by heights. In fact, these Lipschitz curves are vertical lines and locally around the lines the surfaces in the sequence approximate by highly sheeted vertical helicoids. To obtain this additional information, one applies in a neighborhood of each Lipschitz singular curve a blow-up 
argument on the scale of curvature and the uniqueness of the helicoid to prove that the Lipschitz curves are vertical lines. Using the fact that the unit normal field to a codimension-one minimal foliation in a 3-manifold is Lipschitz (Solomon [120]), another blow-up argument has the following consequence.

TheOREM 24 (Regularity of $S(\mathcal{L})$, Meeks [68]). Suppose $\left\{M_{n}\right\}_{n}$ is a ULSC sequence of properly embedded minimal surfaces in a 3-manifold that converges smoothly to a minimal lamination $\mathcal{L}$ outside a locally finite collection of Lipschitz curves $S(\mathcal{L})$ transverse to $\mathcal{L}$. Then $S(\mathcal{L})$ consists of $C^{1,1}$-curves orthogonal to the leaves of $\mathcal{L}$.

Recently, Meeks and Weber [86] have shown that the above $C^{1,1}$-regularity of $S(\mathcal{L})$ is the best possible. They do this by showing that any $C^{1,1}$-curve $\Gamma$ properly embedded in an open set in $\mathbb{R}^{3}$ is the singular set of convergence for some ColdingMinicozzi limit foliation of some neighborhood of itself. In the special case that $\Gamma$ is the unit circle in the $\left(x_{1}, x_{2}\right)$-plane, Meeks and Weber have defined for any $n \in \mathbb{N}$ a complete minimal annulus $H_{n}$ of finite total curvature which contains the circle $\Gamma$; like in the helicoid, the Gauss map of $H_{n}$ turns at a constant rate $2 \pi n$ along its "circle axis" $\Gamma$. Meeks and Weber call the surfaces $H_{n}$ bent helicoids, which have the Weierstrass data: $g(z)=z \frac{z^{n}+i}{z^{n}-i}, d h=\frac{z^{2 n}+1}{z^{n+1}} d z$ defined on $\mathbb{C}-\{0\}$. They prove that there are compact annuli $\bar{H}_{n} \subset H_{n}$ which are embedded and which converge to the foliation $\mathcal{L}$ of $\mathbb{R}^{3}-\left(x_{3}\right.$-axis $)$ by vertical halfplanes containing the $x_{3}$-axis and with singular set of convergence $S(\mathcal{L})=\Gamma$ (for $n=\frac{1}{2}, H_{1 / 2}$ is the double cover of the Meeks's minimal Möbius strip [69] with total curvature $-6 \pi)$.

The regularity Theorem 24 allows one to replace the Lipschitz curves in the next Theorem by vertical lines, which on large balls, yields a parking garage structure with two columns. A little analysis shows that this asymptotic parking garage structure is $(+,-)$-handed, just like in the Riemann minimal case.

Theorem 25 (Colding, Minicozzi [19]). Let $M_{n} \subset B\left(R_{n}\right)$ be a ULSC sequence of embedded minimal planar domains with $\partial M_{n} \subset \partial B\left(R_{n}\right), R_{n} \rightarrow \infty$ and $M_{n} \cap B(2)$ contains a component which is not a disk for any $n$. If $\sup \left|K_{M_{n} \cap B(1)}\right| \rightarrow \infty$, then there exists a subsequence of the $M_{n}$ (denoted in the same way) and two Lipschitz curves $S_{1}, S_{2}: \mathbb{R} \rightarrow \mathbb{R}^{3}$ such that after a rotation of $\mathbb{R}^{3}$ :

1. $x_{3}\left(S_{k}(t)\right)=t$ for all $t \in \mathbb{R}$.

2. Each $M_{n}$ is horizontally locally graphical away from $S_{1} \cup S_{2}$.

3. For each $\alpha \in(0,1), M_{n}-\left(S_{1} \cup S_{2}\right)$ converges in the $C^{\alpha}$-topology to the foliation $\mathcal{L}$ of $\mathbb{R}^{3}$ by horizontal planes.

4. $\sup \left|K_{M_{n} \cap B\left(S_{k}(t), r\right)}\right| \rightarrow \infty$ as $n \rightarrow \infty$, for all $t \in \mathbb{R}$ and $r>0$.

To finish this Section, we next indicate how the one-sided curvature estimate of Colding and Minicozzi can be used to solve the Generalized Nitsche Conjecture. In 1962, Nitsche [95] conjectured that if a minimal surface meets every horizontal plane in a Jordan curve, then it must be a catenoid (he also proved this conjecture with the additional assumption that every horizontal section of the surface is a star shaped curve). In 1993, Meeks and Rosenberg [83] showed that if a properly embedded minimal surface $M \subset \mathbb{R}^{3}$ has at least two ends, then any annular end $E \subset M$ either has finite total curvature or it satisfies the hypotheses of the following conjecture.

Conjecture 7 (Generalized Nitsche Conjecture, Collin's Theorem [25]). Let $E \subset\left\{x_{3} \geq 0\right\}$ be a properly embedded minimal annulus with $\partial E \subset\left\{x_{3}=0\right\}$, such 
that $E$ intersects each plane $\left\{x_{3}=t\right\}, t>0$, in a simple closed curve. Then, $E$ has finite total curvature.

This problem was originally solved by Collin [25] before the Colding-Minicozzi results, with a beautiful and long proof. The argument we present here is a short application of Theorem 23 which can be found in detail in Colding-Minicozzi [21] and in a recent survey by Rosenberg $[\mathbf{1 1 4}]$. Given $\varepsilon \in \mathbb{R}$, we denote by $\mathcal{C}_{\varepsilon}$ the conical region $\left\{x_{3}>\varepsilon \sqrt{x_{1}^{2}+x_{2}^{2}}\right\}$. Conjecture 7 follows directly from the next result.

Theorem 26. [21] There exists $\delta>0$ such that any complete properly embedded minimal annular end $E \subset \mathcal{C}_{-\delta}$ has finite total curvature.

OUtline OF PROOF. The argument starts by showing, for each $\delta>0$, the existence of a sequence $\left\{y_{j}\right\}_{j} \subset E-\mathcal{C}_{\delta}$ with $\left|y_{j}\right| \rightarrow \infty$ (this is done by contradiction: if for a given $\delta>0$ this property fails, then one use $E$ together with the boundary of $\mathcal{C}_{\delta}$ as barriers to construct an end of finite total curvature contained in $\mathcal{C}_{\delta}$, which is clearly impossible). The next step consists of choosing suitable radii $r_{j}>0$ such that the connected component $M_{j}$ of $E \cap B\left(y_{j}, 2 r_{j}\right)$ which contains $y_{j}$ is a disk. Now if $\delta>0$ is sufficiently small in terms of the $\varepsilon$ appearing in the one-sided curvature estimate, one can apply Theorem 23 and conclude a bound for the supremum of the absolute Gaussian curvature of the component $M_{j}^{1}$ of $M_{j} \cap B\left(y_{j}, r_{j}\right)$ which contains $y_{j}$. A Harnack type inequality together with this curvature bound gives a bound for the length of the intrinsic gradient of $x_{3}$ in the intrinsic ball $\mathcal{B}_{j}$ in $M_{j}^{1}$ centered at $y_{j}$ with radius $5 r_{j} / 8$, which in turn implies (by choosing $\varepsilon$ sufficiently small) that $\mathcal{B}_{j}$ is a graph with small gradient over $x_{3}=0$, and one can control a bound by below of the diameter of this graph. This allows to repeat the above argument exchanging $y_{j}$ by a point $y_{j}^{1}$ in $\mathcal{B}_{j}^{1}$ at certain distance from $y_{j}$, and the estimates are carefully done so that the procedure can be iterated to go entirely around a curve $\gamma_{j} \subset E$ whose projection to the $\left(x_{1}, x_{2}\right)$ plane links once around the $x_{3}$-axis. The graphical property of $\gamma_{j}$ implies that either $\gamma_{j}$ can be continued inside $E$ to spiral indefinitely or it closes up with linking number one with the $x_{3}$-axis. The first possibility contradicts that $E$ is properly embedded, and in the second case the topology of $E$ implies that $\partial E \cup \gamma_{j}$ bounds an annulus $E_{j}$. The above gradient estimate gives a linear growth estimate for the length of $\gamma_{j}$ in terms of $\left|y_{j}\right|$, from where the isoperimetric inequality for doubly connected minimal surfaces by Osserman and Schiffer [100] gives a quadratic growth estimate for the area of $E_{j}$. Finally, this quadratic area growth implies the finite total curvature property of $E$, finishing the outline of proof.

\section{Topological aspects of the theory of minimal surfaces.}

Two of the main challenges in the classical theory of minimal surfaces are to decide which noncompact topological types are admissible as properly embedded minimal surfaces in space (Subsection 10.2), and given an admissible topological type in the previous sense, to show that there exists a unique way (up to ambient isotopy) of properly embedding this topological type as a minimal surface in $\mathbb{R}^{3}$ (Subsection 10.1). In the last decade amazing advances have been achieved in both problems, including a final solution to the second problem.

\subsection{Topological classification of properly embedded minimal sur- faces.}


Definition 23. Two properly embedded surfaces in a 3-manifold $N^{3}$ are called ambiently isotopic if one can be deformed to the other by a 1-parameter family of diffeomorphisms of $N^{3}$.

The problem of the topological uniqueness (up to ambient isotopy) of properly embedded minimal surfaces in 3-manifolds has been classically tackled by different authors. In 1970, Lawson [57] showed that two embedded closed diffeomorphic minimal surfaces in $\mathbb{S}^{3}$ are ambiently isotopic. Meeks $[\mathbf{7 0}]$ generalized a key result of Lawson to the case of orientable closed minimal surfaces in a closed 3-manifold with nonnegative Ricci curvature and also proved that any two compact diffeomorphic minimal surfaces embedded in a convex body $B$ in $\mathbb{R}^{3}$, each with boundary a simple closed curve on the boundary of $B$, are ambiently isotopic in $B$ (this result fails for more than one boundary curve, as demonstrated by a counterexample by Hall [42]). Later Meeks, Simon and Yau [85] generalized Lawson's Theorem to the ambient case of $\mathbb{S}^{3}$ with a metric of nonnegative scalar curvature. Returning to $\mathbb{R}^{3}$, Frohman $[\mathbf{3 7}$ ) proved in 1990 that two triply periodic minimal surfaces are always ambiently isotopic. Although published two years later, Meeks and Yau [90] a decade earlier had shown that if $M_{1}, M_{2}$ are properly embedded minimal surfaces in $\mathbb{R}^{3}$ with the same finite topological type, then they are ambiently isotopic.

The essential first point in the proofs of these topological uniqueness results is to obtain a good understanding of the closed complements of the surfaces in the ambient space. This problem of the topological classification of the closed complements and the related uniqueness of the surfaces up to isotopy are closely related to the concept of a Heegaard surface in a 3-manifold, although here we will only deal with the case of the ambient space being $\mathbb{R}^{3}$.

Definition 24. A 3-manifold with boundary is a handlebody if it is homeomorphic to a closed regular neighborhood of a properly embedded one-dimensional CW-complex in $\mathbb{R}^{3}$. A properly embedded surface $M \subset \mathbb{R}^{3}$ is called a Heegaard surface if each of the closed complements of $M$ in $\mathbb{R}^{3}$ are handlebodies.

In 1997, Frohman and Meeks [40] proved that every properly embedded oneended minimal surface in $\mathbb{R}^{3}$ is a Heegaard surface. Additionally, they obtained a topological uniqueness result for Heegaard surfaces in $\mathbb{R}^{3}$ : two Heegaard surfaces of the same genus (possibly infinite) are properly ambiently isotopic. Joining these two results they obtained the following statement.

TheOREM 27 (Frohman, Meeks [40]). Two properly embedded one-ended minimal surfaces in $\mathbb{R}^{3}$ with the same genus are ambiently isotopic.

Furthermore, the topological model for any minimal surface $M$ given in the hypotheses of Theorem 27 , as well as for each of its complements in $\mathbb{R}^{3}$, is easy to describe. Attach $g$ trivial one-handles to the closed lower halfspace $H^{-}$in $\mathbb{R}^{3}$, where $g$ is the genus of $M$. If $g=\infty$, this attaching is performed on neighborhoods of the integer points on the $x_{1}$-axis in $H^{-}$, to obtain a one-periodic Heegaard surface $\Sigma$ in $\mathbb{R}^{3}$. Let $W$ be the handlebody of $\mathbb{R}^{3}$ with boundary $\Sigma$ such that $H^{-} \subset W$. Then, there exists a diffeomorphism $h: \mathbb{R}^{3} \rightarrow \mathbb{R}^{3}$ such that $h(M)$ is the Heegaard surface $\Sigma$ and a prescribed closed complement of $M$ in $\mathbb{R}^{3}$ maps to the handlebody $W$.

To appreciate the power of Theorem 27 , we can consider the singly and doubly periodic Scherk minimal surfaces defined in Subsection 2.5. By Theorem 27, there exists a diffeomorphism of $\mathbb{R}^{3}$ that takes one surface to the other, although they look very different in space. We should also mention here a result by Callahan, 
Hoffman and Meeks [7] that insures that every connected doubly periodic minimal surface has infinite genus and one end. Joining this result with Theorem 27 it follows that any two doubly periodic minimal surfaces in $\mathbb{R}^{3}$ are ambiently isotopic (for example, this applies to the classical P-Schwarz minimal surface and the doubly periodic Scherk surface!).

All these topological uniqueness results are special cases of a recent Theorem by Frohman and Meeks, which represents the final solution to the topological classification problem. It is also based on a deep topological analysis of the complements of a properly embedded minimal surface in space, based on previous work by Freedman [36], and shows what are the topological roles of the ordering on the set of ends given by Theorem 18 and the parity of each middle end defined in Theorem 19 .

Theorem 28 (Topological Classification Theorem, Frohman, Meeks [38]). Two properly embedded minimal surfaces in $\mathbb{R}^{3}$ are ambiently isotopic if and only if there exists a homeomorphism between the surfaces that preserves both the ordering of their ends and the parity of their middle ends.

10.2. Admissible topological types. We will start by discussing noncompact finite topologies, i.e. surfaces with finite genus and finitely many ends. Until the early eighties, no properly embedded minimal surfaces of finite topology other than the plane, the helicoid (both with genus zero, one end) and the catenoid (genus zero, two ends) were known. For a long time, some geometers supported the conjecture that no other examples of finite topology would exist. The discovery in 1982 of a new genus one three-ended example (Costa [28], Hoffman and Meeks [48]) not only disproved this conjecture, but also revitalized enormously the interest of geometers in classical minimal surface theory. Since then, a number of different new examples have appeared, sometimes even coming in multiparameter families $[43,44,45,49,54,123,127]$.

For properly embedded minimal surfaces with finite topology, there is an interesting dichotomy between the one-end case and those surfaces with more that one end: surfaces in this last case always have finite total curvature (Collin's Theorem 3). Only the simplest finite topologies with more than one end have been characterized: López and Ros [63] proved that the unique examples with genus zero and finite topology are the plane and the catenoid, Schoen [118] demonstrated that the catenoid is the unique example with finite genus and two ends, and Costa [29] showed that the examples with genus one and three ends lie inside the one-parameter family of surfaces $\left\{M_{1, a} \mid 0<a<\infty\right\}$ that appear in Subsection 2.5. Today we know many more examples of higher finite topologies and more than one end, and up to this date all known examples support the following conjecture by Hoffman and Meeks.

Conjecture 8 (Finite Topology Conjecture). A connected noncompact (orientable) surface of finite topology, genus $g$ and $r$ ends, $r \neq 2$, can be properly minimally embedded in $\mathbb{R}^{3}$ if and only if $r \leq g+2$.

Recently, Meeks, Pérez and Ros [76] have given the following partial result on the above conjecture.

THEOREM 29. [76] For every nonnegative integer $g$, there exists an integer e $(g)$ such that if $M \subset \mathbb{R}^{3}$ is a properly embedded minimal surface of finite topology and with genus $g$, then the number of ends of $M$ is at most $e(g)$. 
Sketch of The PRoOf. The argument is by contradiction. The failure of the Theorem to hold would produce an infinite sequence $\left\{M_{n}\right\}_{n}$ of properly embedded minimal surfaces with fixed finite genus $g$ and a strictly increasing number of ends. Then one analyzes the nonsimply connected limits of subsequences of $\left\{M_{n}\right\}_{n}$. The key idea used to achieve these limits is to normalize $M_{n}$ by a translation and then a homothety on the scale of topology. What this means is that we assume each surface intersects the closed unit ball centered at the origin in some nonsimply connected component, but that every open ball of radius 1 intersects the surface in simply connected components. Using the uniformly locally simply connected property of $\left\{M_{n}\right\}_{n}$, we prove that its limits are properly embedded nonsimply connected minimal surfaces with genus at most $g$ and possibly infinitely many ends. The infinite topology limits are discarded by an application of either a descriptive Theorem of the geometry of properly embedded minimal surfaces in $\mathbb{R}^{3}$ with finite genus and two limit ends (Meeks, Pérez and Ros [74], see also Theorem 32 below), or a nonexistence Theorem for properly embedded minimal surfaces in $\mathbb{R}^{3}$ with finite genus and one limit end (Meeks, Pérez and Ros [75] or Theorem 31 below). Hence, any possible limit $M$ of a subsequence of $\left\{M_{n}\right\}_{n}$ must be a finite total curvature surface or a helicoid with positive genus at most $g$. A surgery argument allows one to modify the surfaces $M_{n}$ by replacing compact pieces of $M_{n}$ close to the limit $M$ by a finite number of disks, obtaining a new surface $\widetilde{M}_{n}$ with strictly less topology than $M_{n}$ and which is not minimal in the replaced part. A careful study of the replaced parts during the sequence allows one to iterate the process of finding nonsimply connected minimal limits of the modified surfaces. The fact that all the $M_{n}$ have the same genus, allows one to arrive to a stage in the process of producing limits from which all subsequent limits are catenoids. From this point in the proof it is not difficult to find a large integer $n$ such that $M_{n}$ contains a noncompact planar domain $\Omega \subset M_{n}$ whose boundary consists of two convex planar curves $\Gamma_{1}, \Gamma_{2}$ in parallel planes that each separate $M_{n}$ and whose fluxes are orthogonal to the planes that contain $\Gamma_{1}, \Gamma_{2}$. In this setting, the López-Ros deformation defined in Subsection 2.2 (see $[\mathbf{6 3}, \mathbf{1 0 7}]$ ) applies to $\Omega$ giving the desired contradiction.

Since the finite index of a complete minimal surface of finite total curvature can be estimated from above by a function of the degree of its Gauss map (Tysk [125]), Theorem 29 has the following important theoretical consequence.

COROLlaRY 3. For every nonnegative integer $g$, there exists an integer $i(g)$ such that if $M \subset \mathbb{R}^{3}$ is a properly embedded minimal surface with genus $g$ and a finite number of ends greater than 1, then the index of stability of $M$ is at most $i(g)$.

Theorem 29, as well as the related Theorems 31 and 32 below, rely on results by Colding and Minicozzi (see Section 9) to describe the basic local geometry of limits of sequences of properly embedded minimal surfaces with bounded genus and unbounded area.

Concerning one-ended minimal surfaces with finite topology, the model in this class is the helicoid. Recently, Meeks and Rosenberg [80] have proven the following result.

TheOREM 30 (Meeks, Rosenberg [80]). Every properly embedded minimal surface with finite topology and one end has the conformal structure of a compact genus 
$g$ Riemann surface $\bar{M}_{g}$ minus one point, can be analytically represented by meromorphic data on $\bar{M}_{g}$ and is asymptotic to a helicoid. Furthermore, for $g=0$ the only possible examples are the plane and the helicoid.

Sketch of the PRoOf. We will just consider the case where $M$ is simply connected. Let $M$ be a properly embedded simply connected minimal surface in $\mathbb{R}^{3}$ which is not a plane. Consider any sequence of positive numbers $\left\{\lambda_{n}\right\}_{n}$ which decays to zero and let $M(n)=\lambda_{n} M$ be the surface scaled by $\lambda_{n}$. By Theorem 22, a subsequence of these surfaces converges on compact subsets of $\mathbb{R}^{3}$ to a minimal foliation $\mathcal{L}$ of $\mathbb{R}^{3}$ by parallel planes with singular set of convergence $S(\mathcal{L})$. Part of the proof of Theorem 22 depends on a unique extension result for the forming multigraphs, which in our case implies that for $n$ large the almost flat multigraph which starts to form on $M(n)$ near the origin extends all the way to infinity. From here one can deduce that limit foliation $\mathcal{L}$ is independent of the sequence $\left\{\lambda_{n}\right\}_{n}$. After a rotation of $M$ and replacement of the $M(n)$ by a subsequence, we can suppose that the $M(n)$ converge to the foliation $\mathcal{L}$ of $\mathbb{R}^{3}$ by horizontal planes, with singular set of convergence $S(\mathcal{L})$. The property that all surfaces $M(n)$ are simply connected is now crucial in showing that $S(\mathcal{L})$ consists of a single Lipschitz curve $\Gamma$ which intersects each horizontal plane exactly once.

Since the origin is a singular point of convergence, the Lipschitz curve $\Gamma$ passes through the origin and is contained in the solid cone $\mathcal{C}_{\varepsilon}=\left\{x_{3}^{2} \geq \varepsilon^{2}\left(x_{1}^{2}+x_{2}^{2}\right)\right\}$, where $\varepsilon>0$ only depends on the curvature estimate in Theorem 23 . Let $\Delta$ be the solid cylinder $\left\{x_{1}^{2}+x_{2}^{2} \leq 1,\left|x_{3}\right| \leq \varepsilon\right\}$. The two flat horizontal multigraphs $M_{1}(n), M_{2}(n)$ referred to in Theorem 22 intersect the cylindrical sides of $\partial \Delta$ almost orthogonally in two long spiraling arcs which are multigraphs over the unit circle $\mathbb{S}^{1}$ in the $\left(x_{1}, x_{2}\right)$-plane, possibly together with open arcs starting and finishing at the top (resp. bottom) planar disks of $\partial \Delta$ which are graphs over their projections in $\mathbb{S}^{1}$. Both spirals lie on the main compact component $D(n)$ of $M(n) \cap \Delta$. After a small perturbation $\Delta(n)$ of $\Delta$ near the top and bottom boundary disks of $\partial \Delta$ and replacing $\Delta$ by $\Delta(n)$, it can be shown that the boundary of $D(n)$ consists of the two spiraling arcs on the boundary of the cylinder together with two arcs which connect them, one on each of the boundary disks in $\partial \Delta(n)$; in this replacement the new top and bottom disks in $\partial \Delta(n)$ are minimal. Without much difficulty, one can extend the top and bottom disks of $\Delta(n)$ to an almost horizontal minimal foliation of $\Delta(n)$ by graphical minimal disks such that each boundary circle of these disks intersects each spiral curve in $\partial D(n)$ at a single point. Morse theory implies that each leaf of the minimal disk foliation of $\Delta(n)$ intersects $D(n)$ transversely in a simple arc. When $n \rightarrow \infty$, these foliations converge to the restricted foliation $\mathcal{L} \cap \Delta$ by flat horizontal disks. An important consequence of this last statement and of the openness of the Gauss map of the original surface $M$ is that $M$ is transverse to $\mathcal{L}$. This means that the stereographical projection of the Gauss map $g: M \rightarrow \mathbb{C} \cup\{\infty\}$ can be expressed as $g(z)=e^{f(z)}$ for some holomorphic function $f: M \rightarrow \mathbb{C}$.

The next part of the proof is longer and more delicate, and depends in part on a finiteness result for the number of components of minimal graphs over proper domains in $\mathbb{R}^{2}$ with zero boundary values. Through a series of geometric and analytic arguments using the double multigraph convergence of the $M(n)$ to $\mathcal{L}$ outside the cone $\mathcal{C}_{\varepsilon}$, one eventually proves that every horizontal plane in $\mathcal{L}$ intersects $M$ transversely in a single proper arc. Then a straightforward argument using Theorem 12 implies $M$ is recurrent, and thus $M$ is conformally $\mathbb{C}$. The nonexistence 
of points in $M$ with vertical normal vector and the connectedness of its horizontal sections force the height differential to be $d h=d x_{3}+i d x_{3}^{*}=d z$ in a conformal parametrization of $M$. In particular, the third coordinate $x_{3}: \mathbb{C} \rightarrow \mathbb{R}$ is the linear function real part of $z$.

Recall that we have already shown that $g(z)=e^{f(z)}$. If the holomorphic function $f(z)$ is a linear function of the form $a z+b$, then the Weierstrass data $\left(e^{f(z)}, d z\right)$ for $M$ shows that $M$ is an associate surface to the helicoid (see Section 8 for the definition of associate surface). Since none of the nontrivial associate surfaces to the helicoid are injective as mappings, $M$ is a helicoid. Thus, it remains to show that $f(z)$ is linear. The formula (7) for the Gaussian curvature $K$ and a straightforward application of Picard's Theorem imply $f(z)$ is linear if and only if $M$ has bounded curvature. This fact completes the proof of the Theorem in the special case that $K$ is bounded. However, Theorem 22 and a clever blow-up argument on the scale of curvature reduces the proof that $f(z)$ is linear in the general case to the case where $K$ is bounded, and so $M$ is a helicoid. For further details, see $[\mathbf{8 0}]$.

Theorem 30 solves a long standing conjecture about the uniqueness of the helicoid among properly embedded simply connected minimal surfaces in $\mathbb{R}^{3}$. In 1993, Hoffman, Karcher and Wei $[\mathbf{4 6}, \mathbf{4 7}]$ found a torus with one helicoidal end, called since then the genus one helicoid, which has been proved recently to be embedded by Hoffman, Weber and Wolf [51]. Very little is known about genus $g$ helicoids with $g \geq 2$. Computer graphics seem to indicate that higher genus examples probably exist (Traizet — unpublished-, Bobenko [2], Bobenko and Schmies [3]) and Traizet and Weber [124] have given an approach based on the Implicit Function Theorem that could be useful for rigorously proving the existence of an embedded genus $g$ helicoid for every $g$. The Conjecture in this direction, due to Meeks and Rosenberg, is the following one.

Conjecture 9 (One-ended Conjecture). For every nonnegative integer $g$, there exists a unique nonplanar properly embedded minimal surface in $\mathbb{R}^{3}$ with genus $g$ and one end.

Next we deal with properly embedded minimal surfaces with finite genus and infinite topology. Since the number of ends of such a surface $M \subset \mathbb{R}^{3}$ is infinite and the set of ends $\mathcal{E}(M)$ of $M$ is compact (Subsection 2.7), $M$ must have at least one limit end. Up to a rotation, we can assume that the limit tangent plane at infinity of $M$ (see Section 5) is horizontal. A crucial result by Collin, Kusner, Meeks and Rosenberg [26] (Theorem 19) insures that $M$ has no middle limit ends, hence either it has one limit end (this one being the top or the bottom limit end) or both top and bottom ends are the limit ends of $M$, like in a Riemann minimal example. Very recently, Meeks, Pérez and Ros [75] have discarded the one limit end case through the following result.

THEOREM 31. [75] If $M \subset \mathbb{R}^{3}$ is a properly embedded minimal surface with finite genus, then $M$ cannot have exactly one limit end. Furthermore, $M$ is recurrent.

Sketch of the PRoof. Assume $M$ is a properly embedded minimal surface with finite genus and exactly one limit end. After a rotation, we can suppose that $M$ has horizontal tangent plane at infinity and its set of ends, linearly ordered by increasing heights (see the Ordering Theorem 18), is $\mathcal{E}(M)=\left\{e_{1}, e_{2}, \ldots, e_{\infty}\right\}$ 
with the limit end of $M$ being its top end $e_{\infty}$. One first shows that $M$ has a nice asymptotic behavior: each nonlimit end $e_{n} \in \mathcal{E}(M)$ is asymptotic to a graphical annular end $E_{n}$ of a vertical catenoid with negative logarithmic growth $a_{n}$ satisfying $a_{1} \leq \ldots \leq a_{n} \leq \ldots<0$. This is the statement of Theorem 2 in [74]. The next step consists of a detailed analysis of the limits (after passing to a subsequence) of homothetic shrinkings $\left\{\lambda_{n} M\right\}_{n}$, where $\left\{\lambda_{n}\right\}_{n} \subset \mathbb{R}^{+}$is any sequence of numbers decaying to zero; we first show that $\left\{\lambda_{n} M_{n}\right\}_{n}$ is locally simply connected in $H(*)=$ $\left\{x_{3} \geq 0\right\}-\{0\} \subset \mathbb{R}^{3}$. This is a difficult technical part of the proof where the results of Colding-Minicozzi again play a crucial role. We next prove the limits of subsequences of $\left\{\lambda_{n} M_{n}\right\}_{n}$ consist of (possibly singular) minimal laminations $\mathcal{L}$ of $H(*)=\left\{x_{3} \geq 0\right\}-\{0\} \subset \mathbb{R}^{3}$ containing $\partial H(*)$ as a leaf. Then we check that the limit lamination $\mathcal{L}$ is always smooth and that the singular set of convergence $S(\mathcal{L})$ of $\lambda_{n} M$ to $\mathcal{L}$ is empty. In particular, taking $\lambda_{n}=\left\|p_{n}\right\|^{-1}$ where $p_{n}$ is any divergent sequence on $M$, the fact that $S(\mathcal{L})=\varnothing$ for the corresponding limit minimal lamination $\mathcal{L}$ insures that the Gaussian curvature of $M$ decays at least quadratically in terms of the distance function to the origin. Since the Gaussian curvature function times the squared distance to the origin is scale invariant, any leaf $L$ of a limit lamination of $\lambda_{n} M$ for arbitrary $\lambda_{n} \searrow 0$ must also have quadratic decay of its Gaussian curvature function $K_{L}$. Finally, a suitable choice of the sequence $\lambda_{n} \searrow 0$ produces a limit lamination with a nonflat minimal leaf $L$ properly embedded in $H(*)$ of infinite total curvature and such that the scalar flux of the gradient $\nabla x_{3}$ along a certain horizontal section of $L$ is finite. The quadratic decay property for $K_{L}$ together with the existence of a sequence of horizontal planes $\left\{\Pi_{k}\right\}_{k}$ with heights diverging to $+\infty$ such that $L \cap \Pi_{k}$ contains a point with vertical tangent plane (this comes from the fact that $L$ has infinite total curvature) implies that the flux of $\nabla x_{3}$ is unbounded on horizontal sections of $L$. This leads to a contradiction with the invariance of the flux of the divergence free vector field $\nabla x_{3}$ on $L$. This finishes the outline of the proof of the first statement of Theorem 31.

In order to finish the proof, it only remains to check that $M$ is recurrent. If $M$ has exactly one end, then $M$ is conformally a compact Riemann surface minus one point (Theorem 30) and so, $M$ is recurrent. If $M$ has a finite number of ends greater than one, then $M$ has finite total curvature (Theorem 3). By Huber-Osserman's Theorem, $M$ is conformally a compact Riemann surface minus a finite number of points thus it is again recurrent. Finally, if $M$ has infinitely many ends, then $M$ has exactly two limit ends, see the paragraph just before the statement of Theorem 31 . In this situation, Theorem 20 asserts that $M$ is recurrent. This completes our sketch of the proof of Theorem 31 .

In the above sketch of the proof of Theorem 31 we mentioned that any limit lamination $\mathcal{L}$ of $H(*)$ obtained as a limit of (a subsequence of) homothetic shrinkings $\left\{\lambda_{n} M\right\}_{n}$ with $\lambda_{n} \searrow 0$, has no singularities and empty singular set of convergence $S(\mathcal{L})$. To understand why this last property is true, we show that if $\mathcal{L}$ had singularities (in which case $S(\mathcal{L}) \neq \varnothing$ ) or if $S(\mathcal{L})$ were nonempty for a given sequence of shrinkings of $M$, then some smooth leaf of the limit lamination $\mathcal{L}$ that intersects $S(\mathcal{L})$ would be a limit leaf, and hence stable. Our difficulty in discarding this possibility lies in the fact that the stable leaves of $\mathcal{L}$, while perhaps proper in $H(*)$, may not be complete and so, we do not know they must be planes. It is not difficult to prove that the smooth stable leaves in $\mathcal{L}$ in fact satisfy the hypotheses of the next Proposition, and so are in fact planes. Once one has that the smooth stable leaves 
in $\mathcal{L}$ are planes, then the proof of Theorem 22 leads to a contradiction, therefore showing that $S(\mathcal{L})$ is in fact empty. We include the proof of the next Proposition mostly to demonstrate how one can obtain information on the conformal structure of possibly incomplete minimal surfaces by studying conformally related metrics and then how to apply such information to constrain their geometry. Also, the proof of Proposition 4 below gives some new techniques and insights for possibly solving the following famous Conjecture, due to Gulliver and Lawson.

Conjecture 10 (Isolated Singularities Conjecture). There does not exist a properly embedded minimal surface in a punctured ball $B-\{(0,0,0)\}$ whose closure is not a surface at the origin.

Given $\mu>0$ small, we define the upper half cones $\widetilde{C}=\left\{\left(x_{1}, x_{2}, x_{3}\right) \mid x_{3}=\right.$ $\left.\mu \sqrt{x_{1}^{2}+x_{2}^{2}}\right\}$ and $C=\widetilde{C}+(0,0,-1)$. Finally, let $W$ be the component of $\mathbb{R}^{3}-C$ which lies below $C$.

Proposition 4. Let $L$ be a stable orientable minimal surface embedded in $H(*)$, not equal to $\partial H(*)$ and such that $L \cap W$ consists of a nonempty collection of horizontal planar ends. If any proper arc $\alpha:[0, \infty) \rightarrow L$ of finite length satisfies $\lim _{t \rightarrow \infty} \alpha(t)=(0,0,0)$, then $L$ is a horizontal plane.

Proof. Assume $L$ is not a horizontal plane. By the maximum principle, $L \subset$ $\left\{x_{3}>0\right\} \subset \mathbb{R}^{3}$. As $L$ is stable, orientable and is not a plane, Theorem 8 implies that $L$ is not complete. Thus the set $\mathcal{A}$ of proper $\operatorname{arcs} \alpha:[0, \infty) \rightarrow L$ with finite length is nonempty. Proposition 4 will be proven if we show that there exists $\alpha \in \mathcal{A}$ such that $\lim _{t \rightarrow \infty} \alpha(t) \in H(*)-\{(0,0,0)\}$. Reasoning by contradiction, suppose that any $\alpha \in \mathcal{A}$ satisfies $\lim _{t \rightarrow \infty} \alpha(t)=(0,0,0)$.

Consider the complete conformally related metric $\widetilde{g}=\frac{\left(1+x_{3}\right)^{2}}{R^{2}} g$ on $L$, where $g$ is the induced metric on $L$ by the inner product of $\mathbb{R}^{3}$ and $R=\sqrt{x_{1}^{2}+x_{2}^{2}+x_{3}^{2}}$. The Laplace operators and Gaussian curvature functions of $(L, g),(L, \widetilde{g})$ are related by the equations

$$
\widetilde{\Delta}=\frac{R^{2}}{\left(1+x_{3}\right)^{2}} \Delta, \quad \widetilde{K}=\frac{R^{2}}{\left(1+x_{3}\right)^{2}}\left(K-\Delta \ln \frac{1+x_{3}}{R}\right),
$$

where as usual, the notation $\widetilde{\bullet}$ means that the corresponding object $\bullet$ is computed with respect to $\widetilde{g}$ (otherwise it refers to $g$ ). As $\Delta \ln (R)=\frac{2\left(1-|\nabla R|^{2}\right)}{R^{2}} \geq 0$ and $\Delta \ln \left(1+x_{3}\right)=\frac{-\left|\nabla x_{3}\right|^{2}}{\left(1+x_{3}\right)^{2}} \leq 0$, it follows that $\widetilde{K}=\frac{R^{2}}{\left(1+x_{3}\right)^{2}} K+P$ where $P$ is a nonnegative function. As $(L, g)$ is stable, the operator $-\Delta+2 K$ is positive semidefinite on $(L, g)$. Since $-\Delta+K \geq-\Delta+2 K$ and $\frac{R^{2}}{\left(1+x_{3}\right)^{2}}>0$, it follows that $\frac{R^{2}}{\left(1+x_{3}\right)^{2}}(-\Delta+K)$ is also positive semidefinite on $(L, g)$. Since $-\widetilde{\Delta}+\widetilde{K} \geq \frac{R^{2}}{\left(1+x_{3}\right)^{2}}(-\Delta+K)$, we conclude that $-\widetilde{\Delta}+\widetilde{K}$ is positive semidefinite on $(L, \widetilde{g})$.

Let $E$ be the collection of points in the completion of $(L, \widetilde{g})$ corresponding to the annular planar ends of $L$. We claim that the metric completion $\bar{L}$ of $(L, \widetilde{g})$ is exactly $L \cup E$. To see this, let $\alpha:[0, \infty) \rightarrow L$ be a proper arc of finite length on $(L, \widetilde{g})$. If we consider $\alpha$ to lie on $(L, g) \subset\left(\mathbb{R}^{3},<,>\right)$, then the factor $\frac{\left(1+x_{3}\right)^{2}}{R^{2}}$ in the metric $\widetilde{g}$ shows that $\alpha$ eventually lies in the component $W$ of $\mathbb{R}^{3}-C$ below $C$ defined just before the Proposition. Since $\alpha$ is proper, it must lie in one of the annular planar ends of $L$ and so, $\alpha$ diverges to one point in $E$. This proves our claim. 
Note also that the metric $\widetilde{g}$ on $L$ extends to a smooth Riemannian metric $\bar{g}$ on $\bar{L}$ (because the annular ends of $L$ in $E$ are asymptotic to horizontal planes). Since $-\widetilde{\Delta}+\widetilde{K}$ is positive semidefinite on $(L, \widetilde{g})$ and $E \subset \bar{L}$ is a discrete set, we conclude that the extended operator $-\bar{\Delta}+\bar{K}$ is positive semidefinite on $\bar{L}$ (here $\overline{\boldsymbol{\theta}}$ refers to the metric $\bar{g}$ ). As a consequence, the universal covering of $\bar{L}$ is conformally $\mathbb{C}[\mathbf{3 5}]$. Finally, since $\left.x_{3}\right|_{L}$ is a nonconstant positive harmonic function on $(L, \widetilde{g})$ (because it is harmonic on $(L, g))$ and $\left.x_{3}\right|_{L}$ extends smoothly through the points in $E$, we can lift such an extension to the universal covering of $\bar{L}$ as a positive harmonic function on $\mathbb{C}$, hence constant by Liouville's Theorem. In particular, $\left.x_{3}\right|_{L}$ is also constant, which is the desired contradiction.

If a properly embedded minimal surface $M \subset \mathbb{R}^{3}$ has finite genus and infinite topology, then Theorems 19 and 31 imply $M$ has two limit ends which are its top and bottom ends (after a rotation so that the limit tangent plane at infinity of $M$ is horizontal). The classical model in this setting is any of the surfaces in the 1-parameter family of Riemann minimal examples (see Subsection 2.5). In 1998, Meeks, Pérez and Ros [78] proved that these are the unique properly embedded minimal surfaces in $\mathbb{R}^{3}$ with genus zero, infinite topology and infinitely many symmetries. The extension of this characterization by eliminating the hypothesis on the symmetry group constitutes the following conjecture by the same authors.

ConjeCture 11 (Genus zero Conjecture). If $M \subset \mathbb{R}^{3}$ is a properly embedded minimal surface with genus zero and infinitely many ends, then $M$ is a Riemann minimal example.

This conjecture is an active field of research in the last years by Meeks, Pérez and Ros, and in part it has motivated further developments as explained in Theorems 29 and 31 above. Strong partial results have been achieved, such as the following statement.

TheOREM 32. [74] Let $M \subset \mathbb{R}^{3}$ be a properly embedded minimal surface with finite genus, two limit ends and horizontal limit tangent plane at infinity. Then:

1. The middle ends $\left\{e_{n} \mid n \in \mathbb{Z}\right\}$ of $M$ are planar, have heights $\mathcal{H}=$ $\left\{x_{3}\left(e_{n}\right) \mid n \in \mathbb{Z}\right\}$ with $x_{3}\left(e_{n}\right)<x_{3}\left(e_{n+1}\right)$ for all $n \in \mathbb{Z}$ and $\lim _{n \rightarrow \infty} x_{3}\left(e_{n}\right)=\infty$, $\lim _{n \rightarrow-\infty} x_{3}\left(e_{n}\right)=-\infty$.

2. Every horizontal plane sufficiently high or low intersects $M$ in a simple closed curve when its height is not in $\mathcal{H}$ and in a single properly embedded arc when its height is in $\mathcal{H}$.

3. The flux vector of $M$ along any compact (not necessarily connected) horizontal section does not depend on the height of the section. Both the horizontal and the vertical components of this flux vector are not zero. In what follows, we will rescale $M$ so that this vertical component of the flux of $M$ equals 1.

4. $M$ has bounded Gaussian curvature. Furthermore, the maximum asymptotic curvature outside balls $\lim _{r \rightarrow \infty} \sup \left|K_{M-B(r)}\right|$ is bounded from above in terms only of the horizontal component of its flux ${ }^{10}$. In the case $M$ has genus zero,

${ }^{10}$ This means that if $\left\{M_{n}\right\}_{n}$ is a sequence of properly embedded minimal surfaces with fixed finite genus, horizontal limit tangent plane at infinity, the vertical components of the fluxes of the $M_{n}$ along compact horizontal sections are all 1 and the horizontal components of such fluxes are bounded by above, then there are compact balls $B_{n}$ centered at the origin so that $\left\{M_{n}-B_{n}\right\}_{n}$ has uniformly bounded Gaussian curvature. 
the bound on the Gaussian curvature of $M$ depends only on a bound of its horizontal flux.

5. The spacings $S(n)=x_{3}\left(e_{n+1}\right)-x_{3}\left(e_{n}\right)$ between consecutive ends are bounded from above and below (by Corollary 2 in Section 7, the lower bound $2|\lambda|$ depends only on the curvature estimate $\lambda$ for $M$ given in the last point 4).

6. $M$ is quasiperiodic in the following sense. There exists a divergent sequence $V(n) \in \mathbb{R}^{3}$ such that the translated surfaces $M+V(n)$ converge to a properly embedded minimal surface of genus zero, two limit ends and horizontal limit tangent plane at infinity.

7. If $M$ has genus zero, then $M$ has no points with vertical normal vector and the conclusions in point 2 above hold for every horizontal plane in $\mathbb{R}^{3}$.

Using the Shiffman Jacobi function, Meeks, Pérez and Ros have proven the uniqueness of the Riemann minimal examples among properly embedded genus zero minimal surfaces under the hypotheses of Theorem 32, assuming additionally that the horizontal component of the flux along a compact horizontal section is sufficiently small. We will devote Section 11 to a more detailed discussion of this result and to further analysis on the Shiffman Jacobi function.

We finish this Subsection with a brief comment about properly embedded minimal surfaces of infinite genus. The collection of such surfaces with one end is extremely rich. One reason for this is that there are many doubly periodic examples (note that any triply periodic example can be viewed as a doubly periodic one), and as mentioned in Subsection 10.1, every doubly periodic properly embedded minimal surface in $\mathbb{R}^{3}$ has one end (Callahan, Hoffman and Meeks [7]). Besides Theorem 19 on the nonexistence of middle limit ends, very little is known about properly embedded minimal surfaces with infinite genus and infinitely many ends. The first known examples arise from singly periodic surfaces with planar ends and positive genus (Callahan, Hoffman and Meeks [6]), but these examples are better studied as finite total curvature minimal surfaces in the corresponding quotient space. A tentative example of (truly) infinite genus and one limit end might be constructed as follows. Weber and Wolf [127] proved the existence of a sequence $M_{n} \subset \mathbb{R}^{3}$ of properly immersed minimal surfaces of odd genus and $n+2$ horizontal planar ends. Computer graphics pictures indicate that all these surfaces are embedded. Assuming this embeddedness property holds, a suitable normalization of these surfaces should give as a limit a properly embedded minimal surface with a bottom catenoid end, infinitely many middle planar ends and a top limit end. By Theorem 31, this limit surface could not have finite genus.

10.3. Completeness versus properness and minimal laminations. In his beautiful recent survey on minimal surfaces, Harold Rosenberg [114] introduces the subject of his paper through a question asked to him by Andre Haefliger about twenty years ago: "Is there a foliation of $\mathbb{R}^{3}$ by minimal surfaces, other than a foliation by parallel planes?"

Any leaf $L$ of a minimal foliation of $\mathbb{R}^{3}$ is a complete limit leaf and it can be proved to be stable. Now Theorem 8 implies $L$ is a plane (provided $L$ is orientable; but this technical assumption can be supposed after passing to the universal covering, which also turns out to be stable, see the proof of Lemma 1.1 in [80]). Thus the answer to Haefliger's question is no. Immediately one is tempted to extend this question to minimal laminations. 


\section{QUESTION 3. What are the minimal laminations of $\mathbb{R}^{3}$ ?}

There are only two known types of minimal laminations of $\mathbb{R}^{3}$ : a lamination with exactly one leaf which is a properly embedded minimal surface, or a lamination consisting of a closed set of parallel planes. Meeks and Rosenberg [80] have conjectured that these are the unique possible examples. Since every leaf of a minimal lamination of $\mathbb{R}^{3}$ is complete, the above question is closely related to the following one.

QUESTION 4. When is a complete embedded minimal surface $M \subset \mathbb{R}^{3}$ proper?

Given a minimal lamination $\mathcal{L}$ of $\mathbb{R}^{3}$, the function that assigns to each point $p$ in $\mathcal{L}$ the Gaussian curvature of the leaf $L \in \mathcal{L}$ passing through $p$ is continuous in the subspace topology. Since the intersection of $\mathcal{L}$ with any closed ball is compact, we conclude that the intersection of any leaf $L \in \mathcal{L}$ with any ball has Gaussian curvature bounded from below by a constant that only depends on the ball (in other words, $L$ has locally bounded Gaussian curvature). Reciprocally, if $M$ is a complete embedded minimal surface in $\mathbb{R}^{3}$ with locally bounded Gaussian curvature, then the closure $\bar{M}$ of $M$ is a minimal lamination of $\mathbb{R}^{3}$ (Lemma 1.1 in [80]). With this perspective, it is natural to study complete embedded minimal surfaces $M \subset \mathbb{R}^{3}$ with locally bounded Gaussian curvature, as a first stage for possible answers to Questions 3 and 4 . If $M$ is such a minimal surface and it is not proper, then $\bar{M}-M$ may or may not be nonempty; but since $M$ has locally bounded curvature, $\mathcal{L}=\bar{M}$ is a nontrivial minimal lamination of $\mathbb{R}^{3}$ and some leaf $L \in \mathcal{L}$ must be a limit leaf, hence stable. Now an argument similar to the one we used to answer Haefliger's question at the beginning of this Subsection insures that $L$ is a plane; so in this case, $\bar{M}-M$ is always nonempty. This can be stated as follows.

Lemma 4 (Meeks, Rosenberg [80]). Let $M \subset \mathbb{R}^{3}$ be a connected complete embedded minimal surface with locally bounded Gaussian curvature. Then exactly one of the following holds:

1. $M$ is properly embedded in $\mathbb{R}^{3}$.

2. $M$ is properly embedded in an open halfspace, with limit set the boundary plane of this halfspace.

3. $M$ is properly embedded in an open slab, with limit set consisting of the boundary planes of the slab.

It should be mentioned that in a previous work, Xavier [128] proved that a complete, immersed, nonflat minimal surface of bounded curvature in $\mathbb{R}^{3}$ cannot be contained in a halfspace. This result together with Lemma 4 gives a partial answer to Question 4.

Corollary 4. [80] If $M \subset \mathbb{R}^{3}$ is a connected nonflat complete embedded minimal surface with bounded Gaussian curvature, then $M$ is proper.

The next step in the study of complete embedded nonproper minimal surfaces consists of understanding how they accumulate to the limit set described in Lemma 4.

Lemma 5. [80] Let $M \subset \mathbb{R}^{3}$ be a connected complete embedded minimal surface with locally bounded Gaussian curvature. Suppose that $M$ is not proper and let $\Pi$ be a limit plane of $M$. Then, for any $\varepsilon>0$, the closed $\varepsilon$-neighborhood of $\Pi$ intersects $M$ in a path connected set. 
OUtline OF THE PROOF. The argument is by contradiction. Assuming its failure, one can produce a stable minimal surface $\Sigma$ between two components of the intersection of $M$ with the slab $\left\{0<x_{3}<\varepsilon\right\}$ (we do not loss generality by assuming that $\Pi=\left\{x_{3}=0\right\}$ and that $M$ limits to $\Pi$ from above) by the usual barrier construction argument. Since $\Sigma$ satisfies curvature estimates away from its boundary (Theorem 15), we conclude that for sufficiently small $\delta>0$, the orthogonal projection $\pi$ to $\Pi$ restricted to $\Sigma(\delta)=\Sigma \cap\left\{0<x_{3}<\delta\right\}$ is a local diffeomorphism. A topological argument shows that $\left.\pi\right|_{\Sigma(\delta)}$ is in fact bijective, so it is a diffeomorphism. This implies $\Sigma(\delta)$ is properly embedded in the slab $\left\{0 \leq x_{3} \leq \delta\right\}$. Now the argument in the sketch of the proof of Theorem 7 applies to give a contradiction and proves Lemma 5 .

A refinement of the argument in the previous paragraph shows that if $M \subset \mathbb{R}^{3}$ is a connected complete nonproper embedded minimal surface with locally bounded Gaussian curvature that limits to the plane $\Pi=\left\{x_{3}=0\right\}$ from above, then for any $\varepsilon>0$ the Gaussian curvature of $M \cap\left\{0<x_{3} \leq \varepsilon\right\}$ cannot be bounded from below. In other words, there exists a sequence $\left\{p_{n}\right\}_{n} \subset M$ with $x_{3}\left(p_{n}\right) \searrow 0$ and $\left|K_{M}\left(p_{n}\right)\right| \rightarrow \infty$ as $n$ goes to infinity. Such a sequence must diverge in space because $K_{M}$ is locally bounded. If we additionally assume $M$ has finite topology, then an application of the Colding-Minicozzi one-sided curvature estimate (Theorem 23) contradicts that $\left|K_{M}\left(p_{n}\right)\right| \rightarrow \infty$. This is a rough sketch of the proof of the following statement.

THEOREM 33. [80] If $M \subset \mathbb{R}^{3}$ is a connected complete embedded minimal surface in $\mathbb{R}^{3}$ with finite topology and locally bounded Gaussian curvature, then $M$ is proper.

Meeks, Pérez and Ros (Theorem 5 in [74]) have combined the last statement with deeper arguments using the results of Colding and Minicozzi, which let us exchange the finite topology assumption by the weaker hypothesis of finite genus.

THEOREM 34. [74] If $M \subset \mathbb{R}^{3}$ is a connected complete embedded minimal surface in $\mathbb{R}^{3}$ with finite genus and locally bounded Gaussian curvature, then $M$ is proper.

In conclusion, we can state the following descriptive result for minimal laminations of $\mathbb{R}^{3}$.

Theorem 35 (Meeks and Rosenberg [80], Meeks, Pérez and Ros [74]). For a given minimal lamination $\mathcal{L}$ of $\mathbb{R}^{3}$, one of the following possibilities hold.

i) $\mathcal{L}$ has one leaf which consists of a properly embedded minimal surface in $\mathbb{R}^{3}$.

ii) $\mathcal{L}$ has more that one leaf and consists of the disjoint union of a nonempty closed set of parallel planes $\mathcal{P} \subset \mathcal{L}$ together with a collection of complete minimal surfaces of unbounded Gaussian curvature and infinite genus that are properly embedded in the open slabs and halfspaces of $\mathbb{R}^{3}-\mathcal{P}$. Furthermore, each of the open slabs and halfspaces in $\mathbb{R}^{3}-\mathcal{P}$ contains at most one leaf of $\mathcal{L}$, every plane parallel to but different from the planes in $\mathcal{P}$ intersects at most one of the leaves of $\mathcal{L}$ and separates such an intersecting leaf into exactly two components.

To conclude this Subsection, we would like to mention that all the above results depend heavily on the embeddedness and properness assumptions. One sees this in 
part because of the existence of complete immersed minimal surfaces in a ball of $\mathbb{R}^{3}$. The first such an example was a minimal disk constructed by Nadirashvili [94] by a smart application of Runge's Theorem together with the López-Ros deformation. Later on, Martín and Morales [65] gave a bounded complete minimal annulus in $\mathbb{R}^{3}$, and using these techniques together with the Implicit Function Theorem,

López, Martín and Morales $[\mathbf{6 1}, \mathbf{6 0}]$ generalized these complete bounded minimal surfaces to similar examples with any finite topology. As we mentioned in Subsection 3.2, Martín and Morales [64] have recently generalized these results to prove that the interior of any convex, possibly noncompact or nonsmooth, region of $\mathbb{R}^{3}$ admits a proper complete minimal immersion of the unit disk. An interesting question is whether or not such a minimal disk can be embedded.

\section{The Shiffman Jacobi function on properly embedded planar domains.}

Next we explain how an interesting Jacobi function, called the Shiffman function, can help in our attempt to solve Conjecture 11. Let $M \subset \mathbb{R}^{3}$ be a properly embedded minimal surface with genus zero and infinitely many ends. By Theorems 19 and 31, $M$ has two limit ends. After a rotation, we will assume $M$ has horizontal tangent plane at infinity. By Theorem 32, the Gauss map $g$ of $M$ (stereographically projected from the sphere) is a holomorphic function without zeros or poles on $M$. In particular, $M$ intersects each horizontal plane transversally. For minimal surfaces with this last property, Shiffman [119] introduced in 1956 a function that incorporates the curvature variation of the horizontal sections of the surface.

The Shiffman function can be defined locally. Assume that $(g(z), d h=d z)$ is the Weierstrass pair of a minimal surface $M \subset \mathbb{R}^{3}$, where $z$ is a local conformal coordinate in $M$ (in particular, $g$ has no zeros or poles and any minimal surface admits such a local representation around a point with nonvertical normal vector). By (6), the induced metric $d s^{2}$ by the inner product of $\mathbb{R}^{3}$ is $d s^{2}=\Lambda^{2}|d z|^{2}$, where $\Lambda=\frac{1}{2}\left(|g|+|g|^{-1}\right)$. The horizontal level curves $x_{3}=c$ correspond to $z_{c}(y)=c+i y$ in the $z$-plane (here $z=x+i y$ with $x, y \in \mathbb{R}$ and $i^{2}=-1$ ) and the planar curvature of this level curve can be computed as

$$
\kappa_{c}(y)=\left.\left[\frac{|g|}{1+|g|^{2}} \Re\left(\frac{g^{\prime}}{g}\right)\right]\right|_{z=z_{c}(y)},
$$

where the prime stands for derivative with respect to $z$.

Definition 25. We define the Shiffman function of $M$ as

$$
u=\Lambda \frac{\partial \kappa_{c}}{\partial y}=\Im\left[\frac{3}{2}\left(\frac{g^{\prime}}{g}\right)^{2}-\frac{g^{\prime \prime}}{g}-\frac{1}{1+|g|^{2}}\left(\frac{g^{\prime}}{g}\right)^{2}\right],
$$

where $\Im$ stands for imaginary part.

Since $\Lambda$ is a positive function, the zeros of $u$ coincide with the critical points of $\kappa_{c}(y)$. Thus, $u=0$ vanishes identically if and only if $M$ is foliated by pieces of circles and straight lines in horizontal planes. In a posthumously published paper, B. Riemann $[\mathbf{1 1 0}, \mathbf{1 1 1}]$ classified all minimal surfaces with such a foliation property: they reduce to the plane, catenoid, helicoid and the 1-parameter family of surfaces which, since then, have been known as Riemann minimal examples (see Subsection 2.5). 
Coming back to our properly embedded minimal surface $M \subset \mathbb{R}^{3}$ with genus zero, infinitely many ends and horizontal tangent plane at infinity, we now see that a way to solve Conjecture 11 consists of proving that the Shiffman function of $M$ vanishes identically. A crucial property of the Shiffman function is that it satisfies $\Delta u-2 K u=0$ on $M$ (here $K$ is the Gaussian curvature of $M$ ), i.e. $u$ is a Jacobi function. This observation allows one to weaken the condition $u=0$ that characterizes the Riemann minimal examples to the following condition. Recall from Subsection 2.8 that a linear Jacobi function is any function of the type $\langle N, v\rangle$ with fixed $v \in \mathbb{R}^{3}$, where $N$ is the Gauss map of $M$.

LEMma 6. $[\mathbf{1 0 2}, \mathbf{1 0 3}]$ Let $M \subset \mathbb{R}^{3}$ be a properly embedded planar domain with infinitely many ends and transverse to horizontal planes. If the Shiffman function of $M$ is linear, then $M$ is a Riemann minimal example.

Sketch OF PROOF. The argument uses the Montiel-Ros correspondence between Jacobi functions on $M$ and branched minimal immersions with Gauss map $N$ (Theorem 10) to conclude that if the Shiffman function $u=\Im(f)$ of $M$ is linear, then its Jacobi-conjugate function ${ }^{11} u^{*}=\Re(f)$ is also linear, where $f=$ $\frac{3}{2}\left(\frac{g^{\prime}}{g}\right)^{2}-\frac{g^{\prime \prime}}{g}-\frac{1}{1+|g|^{2}}\left(\frac{g^{\prime}}{g}\right)^{2}$. Thus $f=\left\langle N, z_{0}\right\rangle$ for a certain $z_{0} \in \mathbb{C}^{3}$, which in turns implies that $g$ satisfies a differential equation of the type $\left(g^{\prime}\right)^{2}=g\left(\alpha g^{2}+\beta g+\delta\right)$ with $\alpha, \beta, \delta \in \mathbb{C}$. From here it is not difficult to deduce that $M$ is an unbranched covering of a properly embedded minimal torus with two planar ends in a certain quotient of $\mathbb{R}^{3}$ by a translation. Now the classification Theorem by Meeks, Pérez, Ros $[\mathbf{7 8}]$ in the periodic setting applies to finish the proof of the Lemma.

We devote the remainder of this Section to proving that the Shiffman function vanishes on a properly embedded planar domain $M$ with two limit ends and horizontal tangent plane at infinity, provided that the ratio between the horizontal and vertical components of its flux is small enough. To prove this result, we first need to understand the global behavior of the Shiffman function $u$ on such a surface $M$. Using that the Weierstrass pair of $M$ around any of its middle ends is $\left(g(z)=z^{2} t(z), d h=d z\right)$ where $t$ is a holomorphic function of $z$ with $t(0) \neq 0$ and $t^{\prime}(0)=0$ (here $z=0$ corresponds to the puncture, we have assumed that the limit normal vector at the end points to the South Pole of $\mathbb{S}^{2}$ and $t^{\prime}(0)=0$ comes from the fact that the end has no period), a straightforward calculation shows that $u(0)=-\Im\left(\frac{t^{\prime \prime}(0)}{t(0)}\right)$, which implies that $u$ extends smoothly through $z=0$. A similar result holds at the ends where $g$ has a pole. Thus $u$ can be viewed as a continuous function on the conformal cylinder $\bar{M}$ obtained after attaching the middle ends to $M$. By elliptic regularity, $u$ is smooth on $\bar{M}$.

Lemma 7. Let $\delta \in(0,1)$ and let $\Omega \subset \mathbb{R}^{3}$ be a complete noncompact minimal surface with nonempty compact boundary and finite total curvature, such that its Gauss map $N$ satisfies $N_{3}=\left\langle N, e_{3}\right\rangle \geq 1-\delta$ in $\Omega$. Then, for every bounded Jacobi function $v$ on $\Omega$,

$$
(1-\delta) \sup _{\Omega}|v| \leq \sup _{\partial \Omega}|v|
$$

${ }^{11}$ Two Jacobi functions $v, v^{*}$ on $M$ are called Jacobi-conjugate if there exists a globally defined complex solution $f$ of the Jacobi equation $\Delta f-2 K f=0$ on $M$ such that $v=\Re(f)$ and $v^{*}=\Im(f)$. 
Proof. Since $\Omega$ has finite total curvature, $\Omega$ compactifies after attaching its ends to a compact Riemann surface $\bar{\Omega}$ with boundary. Since $v$ is bounded on $\Omega$, $v$ extends smoothly across the punctures to a Jacobi function on $\bar{\Omega}$. We will let $a=\sup _{\partial \Omega}|v|$. Since $N_{3} \geq 1-\delta>0$ in $\Omega$ and $N_{3}$ is Jacobi, we conclude that $\Omega$ is strictly stable and so, $a>0$. Now, $v+\frac{a}{1-\delta} N_{3} \geq 0$ in $\partial \Omega$ and $v+\frac{a}{1-\delta} N_{3}$ is Jacobi on $\Omega$, thus by stability $v+\frac{a}{1-\delta} N_{3} \geq 0$ in $\Omega$. Analogously, $v-\frac{a}{1-\delta} N_{3} \leq 0$ in $\partial \Omega$, hence $v-\frac{a}{1-\delta} N_{3} \leq 0$ in $\Omega$. These inequalities together with $N_{3} \leq 1$ give $|v| \leq \frac{a}{1-\delta}$ in $\Omega$, as desired.

Theorem 32 implies that each properly embedded minimal planar domain $M$ with infinite topology and horizontal tangent plane at infinity has a well-defined flux vector, which is the flux of $M$ along any compact horizontal section, and this vector is neither vertical nor horizontal. In the sequel, we will normalize $M$ by a homothety so that its flux vector has the form $(F(M), 1) \in \mathbb{C} \times \mathbb{R} \equiv \mathbb{R}^{3}$. Let $\mathcal{S}$ be the space of all properly embedded minimal planar domains with infinitely many ends, horizontal tangent plane at infinity and vertical component of the flux equal to one.

Theorem 36 (Meeks, Pérez, Ros [102]). There exists $\varepsilon>0$ such that if $M \in \mathcal{S}$ has $|F(M)|<\varepsilon$, then $M$ is a Riemann minimal example.

Proof. We will present here a different proof from the one in [102]. By contradiction, assume we have a sequence $\left\{M_{n}\right\}_{n} \subset \mathcal{S}$ with $F\left(M_{n}\right) \rightarrow 0$, and none of the $M_{n}$ is a Riemann minimal example. Point 4 in Theorem 32 insures that $\left\{M_{n}\right\}_{n}$ has uniformly bounded Gaussian curvature. A suitable modification of the arguments in the proof of Lemma 3 in [78] can be used to show that as $n \rightarrow \infty$, the surfaces $M_{n}$ become arbitrarily close to an infinite discrete collection of larger and larger translated pieces of a vertical catenoid with flux $e_{3}=(0,0,1)$ joined by flatter and flatter graphs containing the ends of $M_{n}$. For each $n$, let $\bar{M}_{n}$ be the conformal cylinder obtained by attaching the middle ends to $M_{n}$, and let $u_{n}$ be the Shiffman function of $M_{n}$.

Assertion 1. For $n \in \mathbb{N}$ large enough, $u_{n}$ is bounded on $\bar{M}_{n}$.

Proof of Assertion 1. Suppose that the Assertion fails. To simplify the notation, we will denote the surface $M_{n}$ only by $M$, and think of $M$ as being arbitrarily close to pieces of translated catenoids and flat graphs as above. The failure of the Assertion allows us to find a subsequence of points $p_{k} \in M$ such that $\left|u\left(p_{k}\right)\right| \rightarrow \infty$ as $k$ goes to $\infty$ (here $u$ is the Shiffman function of $M$ ). Note that $\left\{p_{k}\right\}_{k}$ must be a divergent sequence in height (otherwise we contradict that $u$ stays bounded at any middle end of $M)$. By Theorem $32,\left\{M-p_{k}\right\}_{k}$ is a sequence of properly embedded minimal surfaces in $\mathbb{R}^{3}$ with uniform bounds for the Gaussian curvature and area. After passing to a subsequence, $M-p_{k}$ converges to a properly embedded minimal surface $M_{\infty} \in \mathbb{R}^{3}$ with $0 \in M_{\infty}$.

First suppose that the value of the Gauss map $N$ of $M$ at $p_{k}$ does not converge to vertical as $k \rightarrow \infty$. Then, a suitable modification of the arguments in the proof of Lemma 2 in [78] insures that $M_{\infty}$ cannot be flat. This implies the sequence $\left\{M-p_{k}\right\}_{k}$ converges smoothly to $M_{\infty}$ with multiplicity one, and a lifting argument shows that $M_{\infty}$ has genus zero. Since $M_{\infty}$ has no points with vertical normal vector (which comes from the open mapping Theorem applied to the Gauss map of $M_{\infty}$ ), $M_{\infty}$ must have a well-defined Shiffman function $u_{\infty}$, which is nothing but the limit 
of $u_{k}(q)=u\left(q+p_{k}\right), q \in M-p_{k}$. In particular $u\left(p_{k}\right)$ converges to $u_{\infty}(0)$, which is a contradiction.

Now assume that, after extracting a subsequence, $N\left(p_{k}\right)$ converges to $e_{3}$ as $k \rightarrow \infty$. By taking $k$ sufficiently large, we conclude that $p_{k}$ lies in one of the almost flat graphs $\Omega \subset M=M_{n}$ that joins two consecutive catenoids forming. This contradicts Lemma 7 , since $|u|$ is arbitrarily small in $\partial \Omega$ but $\left|u\left(p_{k}\right)\right| \rightarrow \infty$. Now Assertion 1 is proved.

In the sequel, we will work with $n$ large so that Assertion 1 holds. Note that for fixed $n$, the function $\left|u_{n}\right|$ needs not attain its maximum on $M_{n}$, but in that case we can exchange each $M_{n}$ by a limit of suitable translations of $M_{n}$ so that the Shiffman function in absolute value reaches its maximum on this limit. Since the flux of a surface in $\mathcal{S}$ does not change under translations, we do not loss generality by assuming that for all $n$ large, $\left|u_{n}\right|$ attains its maximum at a point $p_{n} \in M_{n}$. We now define $v_{n}=\frac{1}{\left|u_{n}\left(p_{n}\right)\right|} u_{n}$.

Take a sequence $\{\delta(n)\}_{n} \subset(0,1)$ converging to 1 . For $n$ large, let $C_{n} \subset M_{n}$ be one of the connected components of $\left\langle N_{n}, e_{3}\right\rangle^{-1}[-\delta(n), \delta(n)]$ which contains $p_{n}$ or is adjacent to a horizontal graphical region containing $p_{n}$. By our previous arguments, $C_{n}$ is arbitrarily close to a translated image of the intersection of a vertical catenoid $C_{\infty}$ of vertical flux $e_{3}$ centered at the origin with a ball of arbitrarily large radius also centered at the origin.

Assertion 2. $\left\{\sup _{C_{n}}\left|v_{n}\right|\right\}_{n}$ tends to zero as $n \rightarrow \infty$.

Proof of Assertion 2. Since $\left\{\left.v_{n}\right|_{C_{n}}\right\}_{n}$ is a bounded sequence of Jacobi functions on the $C_{n}$ and suitable translations of the $C_{n}$ converge to the catenoid $C_{\infty}$, it is not difficult to check that a subsequence of $\left\{\left.v_{n}\right|_{C_{n}}\right\}_{n}$ (denoted in the same way) converges to a bounded Jacobi function on $C_{\infty}$. Since bounded Jacobi functions on a catenoid are linear, we conclude that $\left\{\left.v_{n}\right|_{C_{n}}\right\}_{n}$ converges to a linear Jacobi function $v$ on $C_{\infty}$ (or by identifying $C_{\infty}$ with the sphere $\mathbb{S}^{2}$ through its Gauss map, we can see $v$ as a linear function on $\mathbb{S}^{2}$ ). We now check that $v$ is identically zero on $\mathbb{S}^{2}$.

By contradiction, suppose $v$ is not identically zero on $\mathbb{S}^{2}$. Recall that the Shiffman function $\left.u_{n}\right|_{C_{n}}$ measures the derivative of the curvature of each planar section of $C_{n}$ with respect to a certain parameter times a positive function. By the Four Vertex Theorem, each horizontal section of $C_{n}$ contains at least four zeros of $u_{n}$ and so, also at least four zeros of $v_{n}$. Since horizontal sections of the $C_{n}$ (suitably translated) converge to horizontal sections of $C_{\infty}$ and any nontrivial linear function on $\mathbb{S}^{2}$ has at most two zeros on each horizontal section (with a possible exceptional horizontal section if the linear function is the vertical coordinate, but this does not affect to our argument by taking a different horizontal section), we conclude that at least two zeros of $v_{n}$ in a certain horizontal section must collapse into a zero of $v$, hence the gradient of $v$ will vanish at such a collapsing zero. But the gradient of a nontrivial linear function on $\mathbb{S}^{2}$ never vanishes at a zero of the function. This contradiction proves Assertion 2.

Recall that $\left|v_{n}\left(p_{n}\right)\right|=1$ for all $n$. By Assertion 2, $N_{n}\left(p_{n}\right)$ must converge to the vertical or equivalently, $p_{n}$ must lie in one of the graphical components of the complement of all the catenoidal pieces in $M_{n}$, a noncompact minimal graph which we will denote by $\Omega_{n}$. Note that $\Omega_{n}$ is a graph over an unbounded domain in the plane $\left\{x_{3}=0\right\}, \partial \Omega_{n}$ consists of two almost-circular almost-horizontal curves with $\left.\left\langle N_{n}, e_{3}\right\rangle\right|_{\partial \Omega_{n}}= \pm \delta(n)$ and $\Omega_{n}$ contains exactly one end of $M_{n}$. Hence we can apply 
Lemma 7 to the minimal surface $\Omega_{n}$ and to the bounded Jacobi function $\left.v_{n}\right|_{\Omega_{n}}$, contradicting that $\left.v_{n}\right|_{\partial \Omega_{n}}$ converges to zero (Assertion 2) but $\left|v\left(p_{n}\right)\right|=1$. This contradiction finishes the proof of the Theorem.

The proof of Theorem 36 that appears in $[\mathbf{1 0 2}]$ is based on the relationship between the Shiffman function and the index form $Q(v, v)=\int_{\Omega}\left(|\nabla v|^{2}+2 K v^{2}\right)$ on any connected complement $\Omega$ of $\{|g|=1\}$ in a planar domain $M \in \mathcal{S}$. A similar technique with the nodal domains of a linear function can be applied to conclude the following description of all bounded Jacobi functions on any Riemann minimal example.

Theorem 37 (Meeks, Pérez, Ros [77]). Let $M \subset \mathbb{R}^{3}$ be a Riemann minimal example. Then any bounded Jacobi function on $M$ is linear.

The above Theorem plays a central role in our program to prove that any finite genus limit end of a properly embedded minimal surface converges exponentially quickly to a limit end of one of the Riemann minimal examples. The Theorem should also be useful in proving that the moduli space of genus 1 properly embedded minimal surfaces with horizontal limit tangent plane at infinity and two limit ends is an open interval parametrized by the ratio of the horizontal and vertical components of the flux, in the same way that the Riemann examples are parametrized, and for finite $g \geq 2$, the moduli space of the genus $g$ examples with two limit ends has an infinite number of path components.

\section{References}

[1] S. Bernstein. Uber ein geometrisches theorem und seine anwendung auf die partiellen differentialglechungen vom elliptischen typus. Math. Z., 26:551-558, 1927.

[2] A. I. Bobenko. Helicoids with handles and Baker-Akhiezer spinors. Math. Z., (1):9-29, 1998.

[3] A. I. Bobenko and M. Schmies. Computer graphics experiments for helicoids with handles. Personal communication.

[4] O. Bonnet. Mémoire sur l'emploi d'un nouveau systeme de variables dans l'etude des surfaces courbes. J. Mathemém. p. appl., 2:153-266, 1860.

[5] E. Calabi. Quelques applications de l'Analyse complexe aux surfaces d'Aire minima. In Topics in Complex Manifolds, pages 59-81. Les Presses de l'Université de Montréal, 1967. H. Rossi, editor.

[6] M. Callahan, D. Hoffman, and W. H. Meeks III. Embedded minimal surfaces with an infinite number of ends. Invent. Math., 96:459-505, 1989.

[7] M. Callahan, D. Hoffman, and W. H. Meeks III. The structure of singly-periodic minimal surfaces. Invent. Math., 99:455-481, 1990.

[8] E. Catalan. Sur les surfaces réglées dont l'aire est un minimum. J. Mathem. p. appl., 7:203211, 1842 .

[9] I. Chavel. Riemannian Geometry: a modern introduction. Cambridge University Press, 1993.

[10] J. Cheeger and D. G. Ebin. Comparison theorems in Riemannian Geometry. North-Holland Mathematical Library, Vol. 9., 1975.

[11] H. I. Choi and R. Schoen. The space of minimal embeddings of a surface into a threedimensional manifold of positive Ricci curvature. Invent. Math., 81:387-394, 1985.

[12] T. Choi, W. H. Meeks III, and B. White. A rigidity theorem for properly embedded minimal surfaces in $\mathbb{R}^{3}$. J. of Differential Geometry, 32:65-76, 1990.

[13] T. H. Colding and W. P. Minicozzi II. Embedded minimal disks: proper versus nonproper - global versus local. Transactions of A.M.S. (to appear).

[14] T. H. Colding and W. P. Minicozzi II. An excursion into geometric analysis. J. Diff. Geom.

[15] T. H. Colding and W. P. Minicozzi II. The space of embedded minimal surfaces of fixed genus in a 3-manifold I; Estimates off the axis for disks. Annals of Math. (to appear). 
[16] T. H. Colding and W. P. Minicozzi II. The space of embedded minimal surfaces of fixed genus in a 3-manifold II; Multi-valued graphs in disks. Annals of Math. (to appear).

[17] T. H. Colding and W. P. Minicozzi II. The space of embedded minimal surfaces of fixed genus in a 3-manifold III; Planar domains. Annals of Math. (to appear).

[18] T. H. Colding and W. P. Minicozzi II. The space of embedded minimal surfaces of fixed genus in a 3-manifold IV; Locally simply-connected. Annals of Math. (to appear).

[19] T. H. Colding and W. P. Minicozzi II. The space of embedded minimal surfaces of fixed genus in a 3-manifold V; Fixed genus. in preparation.

[20] T. H. Colding and W. P. Minicozzi II. Minimal surfaces. Courant Institute of Mathematical Sciences Lecture Notes 4, 1999.

[21] T. H. Colding and W. P. Minicozzi II. Complete properly embedded minimal surfaces in $\mathbb{R}^{3}$. Duke Math. J., 107:421-426, 2001.

[22] T. H. Colding and W. P. Minicozzi II. Estimates for parametric elliptic integrands. Int. Math. Res. Not., (6):291-297, 2002.

[23] T. H. Colding and W. P. Minicozzi II. Multi-valued minimal graphs and properness of disks. International Mathematical Research Notices, (21):1111-1127, 2002.

[24] T. H. Colding and W. P. Minicozzi II. Disks that are double spiral staircases. Notices of A.M.S., 50(3):327-339, 2003.

[25] P. Collin. Topologie et courbure des surfaces minimales de $\mathbb{R}^{3}$. Annals of Math. 2nd Series, 145-1:1-31, 1997.

[26] P. Collin, R. Kusner, W. H. Meeks III, and H. Rosenberg. The geometry, conformal structure and topology of minimal surfaces with infinite topology. Preprint.

[27] C. Costa. Imersöes minimas en $\mathbb{R}^{3}$ de gênero un e curvatura total finita. PhD thesis, IMPA, Rio de Janeiro, Brasil, 1982.

[28] C. Costa. Example of a complete minimal immersion in $\mathbb{R}^{3}$ of genus one and three embedded ends. Bull. Soc. Bras. Mat., 15:47-54, 1984.

[29] C. Costa. Uniqueness of minimal surfaces embedded in $\mathbb{R}^{3}$ with total curvature $12 \pi$. J. of Differential Geometry, 30(3):597-618, 1989.

[30] C. Costa. Classification of complete minimal surfaces in $\mathbb{R}^{3}$ with total curvature $12 \pi$. Invent. Math., 105(2):273-303, 1991.

[31] M. do Carmo and C. K. Peng. Stable minimal surfaces in $\mathbb{R}^{3}$ are planes. Bulletin of the AMS, 1:903-906, 1979.

[32] H. M. Farkas and I. Kra. Riemann Surfaces. Number 72 in Graduate texts in mathematics. Springer-Verlag, 1980.

[33] H. Federer. Geometric measure theory. Springer Verlag, Berlin-Heidelberg, New York, 1969.

[34] D. Fischer-Colbrie. On complete minimal surfaces with finite Morse index in 3-manifolds. Invent. Math., 82:121-132, 1985.

[35] D. Fischer-Colbrie and R. Schoen. The structure of complete stable minimal surfaces in 3-manifolds of non-negative scalar curvature. Comm. on Pure and Appl. Math., 33:199-211, 1980.

[36] M. Freedman. An unknotting result for complete minimal surfaces in $\mathbb{R}^{3}$. Invent. Math, 109(1):41-46, 1992.

[37] C. Frohman. The topological uniqueness of triply-periodic minimal surfaces in $\mathbb{R}^{3}$. J. of Differential Geometry, 31:277-283, 1990.

[38] C. Frohman and W. H. Meeks III. The topological classification of minimal surfaces in $\mathbb{R}^{3}$. Preprint.

[39] C. Frohman and W. H. Meeks III. The ordering theorem for the ends of properly embedded minimal surfaces. Topology, 36(3):605-617, 1997.

[40] C. Frohman and W. H. Meeks III. The topological uniqueness of complete one-ended minimal surfaces and Heegaard surfaces in $\mathbb{R}^{3}$. J. of the Amer. Math. Soc., 10(3):495-512, 1997.

[41] A. Grigor'yan. Analytic and geometric background of recurrence and non-explosion of Brownian motion on Riemannian manifolds. Bull. of A.M.S, 36(2):135-249, 1999.

[42] P. Hall. Two topological examples in minimal surface theory. J. of Differential Geometry, 19:475-481, 1984.

[43] D. Hoffman. The computer-aided discovery of new embedded minimal surfaces. Mathematical Intelligencer, 9(3):8-21, 1987. 
[44] D. Hoffman and H. Karcher. Complete embedded minimal surfaces of finite total curvature. In R. Osserman, editor, Encyclopedia of Mathematics, Vol. 90, Geometry V, pages 5-93. Springer Verlag, 1997.

[45] D. Hoffman, H. Karcher, and W. H. Meeks III. One-parameter families of embedded complete minimal surfaces of finite topology. GANG preprint in preparation.

[46] D. Hoffman, H. Karcher, and F. Wei. Adding handles to the helicoid. Bulletin of the AMS, New Series, 29(1):77-84, 1993.

[47] D. Hoffman, H. Karcher, and F. Wei. The genus one helicoid and the minimal surfaces that led to its discovery. In Global Analysis and Modern Mathematics. Publish or Perish Press, 1993. K. Uhlenbeck, editor, p. 119-170.

[48] D. Hoffman and W. H. Meeks III. A complete embedded minimal surface in $\mathbb{R}^{3}$ with genus one and three ends. J. of Differential Geometry, 21:109-127, 1985.

[49] D. Hoffman and W. H. Meeks III. Embedded minimal surfaces of finite topology. Annals of Math., 131:1-34, 1990.

[50] D. Hoffman and W. H. Meeks III. The strong halfspace theorem for minimal surfaces. Invent. Math., 101:373-377, 1990.

[51] D. Hoffman, M. Weber, and M. Wolf. The existence of the genus-one helicoid. Preprint.

[52] A. Huber. On subharmonic functions and differential geometry in the large. Comment. Math. Helvetici, 32:181-206, 1957.

[53] L. Jorge and W. H. Meeks III. The topology of complete minimal surfaces of finite total Gaussian curvature. Topology, 22(2):203-221, 1983.

[54] N. Kapouleas. Complete embedded minimal surfaces of finite total curvature. J. Differential Geom., 47(1):95-169, 1997.

[55] N. Korevaar, R. Kusner, and B. Solomon. The structure of complete embedded surfaces with constant mean curvature. J. of Differential Geometry, 30:465-503, 1989.

[56] R. Langevin and H. Rosenberg. A maximum principle at infinity for minimal surfaces and applications. Duke Math. J., 57(3):819-828, 1988.

[57] H. B. Lawson. The unknottedness of minimal embeddings. Invent. Math., 11:183-187, 1970.

[58] H. B. Lawson, Jr. Lectures on Minimal Submanifolds. Publish or Perish Press, Berkeley, 1971.

[59] H. Lazard-Holly and W. H. Meeks III. The classification of embedded doubly-periodic minimal surfaces of genus zero. Invent. Math., 143:1-27, 2001.

[60] F. J. López, F. Martín, and S. Morales. Complete nonorientable minimal surfaces in a ball of $\mathbb{R}^{3}$. Preprint.

[61] F. J. López, F. Martín, and S. Morales. Adding handles to Nadirashvili's surfaces. Journal of Diff. Geom., 60:155-175, 2002.

[62] F. J. López and J. Pérez. Parabolicity and Gauss map of minimal surfaces. Indiana J. of Math., 52(4):1017-1026, 2003.

[63] F. J. López and A. Ros. On embedded complete minimal surfaces of genus zero. J. of Differential Geometry, 33(1):293-300, 1991.

[64] F. Martin and S. Morales. Complete and proper minimal surfaces in convex bodies. Preprint.

[65] F. Martin and S. Morales. On the asymptotic behavior of a complete bounded minimal surface in $\mathbb{R}^{3}$. Trans. of the A.M.S. (to appear).

[66] W. H. Meeks III. The geometry and topology of singly-periodic minimal surfaces. to appear in the Asian J. of Math.

[67] W. H. Meeks III. Global problems in classical minimal surface theory. To appear in the Clay Minimal Surface Conference Proceedings.

[68] W. H. Meeks III. The regularity of the singular set in the Colding and Minicozzi lamination theorem. To appear in Duke Math. J.

[69] W. H. Meeks III. The classification of complete minimal surfaces with total curvature greater than $-8 \pi$. Duke Math. J., 48:523-535, 1981.

[70] W. H. Meeks III. The topological uniqueness of minimal surfaces in three-dimensional Euclidean space. Topology, 20:389-410, 1981.

[71] W. H. Meeks III. The theory of triply-periodic minimal surfaces. Indiana Univ. Math. J., 39(3):877-936, 1990.

[72] W. H. Meeks III. The geometry, topology, and existence of periodic minimal surfaces. Proceedings of Symposia in Pure Math., 54:333-374, 1993. Part I. 
[73] W. H. Meeks III and J. Pérez. Recent advances in classical minimal surface theory. Work in progress.

[74] W. H. Meeks III, J. Pérez, and A. Ros. The geometry of minimal surfaces of finite genus I; curvature estimates and quasiperiodicity. To appear in J. Diff. Geom.

[75] W. H. Meeks III, J. Pérez, and A. Ros. The geometry of minimal surfaces of finite genus II; nonexistence of one limit end examples. To appear in Invent. Math.

[76] W. H. Meeks III, J. Pérez, and A. Ros. The geometry of minimal surfaces of finite genus III; bounds on the topology and index of classical minimal surfaces. Preprint.

[77] W. H. Meeks III, J. Pérez, and A. Ros. The geometry of minimal surfaces of finite genus IV; Jacobi fields and uniqueness for small flux. Work in progress.

[78] W. H. Meeks III, J. Pérez, and A. Ros. Uniqueness of the Riemann minimal examples. Invent. Math., 131:107-132, 1998.

[79] W. H. Meeks III and H. Rosenberg. Maximum principles at infinity with applications to minimal and constant mean curvature surfaces. Preprint.

[80] W. H. Meeks III and H. Rosenberg. The uniqueness of the helicoid and the asymptotic geometry of properly embedded minimal surfaces with finite topology. To appear in Annals of Math.

[81] W. H. Meeks III and H. Rosenberg. The global theory of doubly periodic minimal surfaces. Invent. Math., 97:351-379, 1989.

[82] W. H. Meeks III and H. Rosenberg. The maximum principle at infinity for minimal surfaces in flat three-manifolds. Comment. Math. Helvetici, 65:255-270, 1990.

[83] W. H. Meeks III and H. Rosenberg. The geometry and conformal structure of properly embedded minimal surfaces of finite topology in $\mathbb{R}^{3}$. Invent. Math., 114:625-639, 1993.

[84] W. H. Meeks III and H. Rosenberg. The geometry of periodic minimal surfaces. Comment. Math. Helvetici, 68:538-578, 1993.

[85] W. H. Meeks III, L. Simon, and S. T. Yau. The existence of embedded minimal surfaces, exotic spheres and positive Ricci curvature. Annals of Math., 116:221-259, 1982.

[86] W. H. Meeks III and M. Weber. Existence of bent helicoids and the geometry of the singular set in the Colding-Minicozzi lamination theorem. Preprint.

[87] W. H. Meeks III and M. Wolf. Solution to the finite flux conjecture in dimension two. Work in progress.

[88] W. H. Meeks III and S. T. Yau. The classical Plateau problem and the topology of threedimensional manifolds. Topology, 21(4):409-442, 1982.

[89] W. H. Meeks III and S. T. Yau. The existence of embedded minimal surfaces and the problem of uniqueness. Math. Z., 179:151-168, 1982.

[90] W. H. Meeks III and S. T. Yau. The topological uniqueness of complete minimal surfaces of finite topological type. Topology, 31(2):305-316, 1992.

[91] S. Montiel and A. Ros. Schrödinger operators associated to a holomorphic map. In Global Differential Geometry and Global Analysis (Berlin, 1990), volume 1481 of Lecture Notes in Mathematics, pages 147-174. Springer-Verlag, 1990.

[92] S. Morales. On the existence of a proper minimal surface in $\mathbb{R}^{3}$ with the conformal type of a disk. To appear in GAFA.

[93] C. B. Morrey. The problem of Plateau in a Riemannian manifold. Annals of Math., 49:807851, 1948.

[94] N. Nadirashvili. Hadamard's and Calabi-Yau's conjectures on negatively curved and minimal surfaces. Invent. Math., 126(3):457-465, 1996.

[95] J. C. C. Nitsche. A characterization of the catenoid. J. of Math. Mech., 11:293-302, 1962.

[96] J. C. C. Nitsche. A new uniqueness theorem for minimal surfaces. Arch. Rat. Mech. Anal., $52: 319-329,1973$.

[97] J. C. C. Nitsche. Lectures on Minimal Surfaces, volume 1. Cambridge University Press, 1989.

[98] R. Osserman. Global properties of minimal surfaces in $E^{3}$ and $E^{n}$. Annals of Math., 80(2):340-364, 1964.

[99] R. Osserman. A Survey of Minimal Surfaces. Dover Publications, New York, 2nd edition, 1986.

[100] R. Osserman and M. Schiffer. Doubly-connected minimal surfaces. Arch. Rat. Mech. Anal., 58:285-307, 1975. 
[101] J. Pérez. Parabolicity and minimal surfaces. To appear in the Proceedings of the Conference on Global Theory of Minimal Surfaces, Berkeley 2001.

[102] J. Pérez. Uniqueness of the Riemann minimal surfaces. To appear in the Proceedings of the Conference on Global Theory of Minimal Surfaces, Berkeley 2001.

[103] J. Pérez. On singly-periodic minimal surfaces with planar ends. Trans. of the A.M.S., 6:2371-2389, 1997.

[104] J. Pérez. A rigidity theorem for periodic minimal surfaces. Comm. in Analysis and Geom., 7(1):95-104, 1999.

[105] J. Pérez and A. Ros. Some uniqueness and nonexistence theorems for embedded minimal surfaces. Math.Ann., 295(3):513-525, 1993.

[106] J. Pérez and A. Ros. The space of properly embedded minimal surfaces with finite total curvature. Indiana Univ. Math. J., 45(1):177-204, 1996.

[107] J. Pérez and A. Ros. Properly embedded minimal surfaces with finite total curvature. In The Global Theory of Minimal Surfaces in Flat Spaces-LN M-1775, pages 15-66. Springer Verlag, 1999. G. P. Pirola, editor.

[108] J. Pitts and H. Rubenstein. Applications of minimax to minimal surfaces and the topology of three-manifolds. In Proceedings for the Center for Mathematical Analysis, pages 137-170, Canberra, Australia, 1987. Australian National University.

[109] A. V. Pogorelov. On the stability of minimal surfaces. Soviet Math. Dokl., 24:274-276, 1981.

[110] B. Riemann. Über die Fläche vom kleinsten Inhalt bei gegebener Begrenzung. Abh. Königl, d. Wiss. Göttingen, Mathem. Cl., 13:3-52, 1867. K. Hattendorf, editor.

[111] B. Riemann. Ouevres Mathématiques de Riemann. Gauthiers-Villars, Paris, 1898.

[112] A. Ros. Compactness of spaces properly embedded minimal surfaces with finite total curvature. Indiana Univ. Math. J., 44(1):139-152, 1995.

[113] A. Ros. The Gauss map of minimal surfaces. In Differential Geometry, Valencia 2001, Proceedings of the conference in honour of Antonio M. Naveira, pages 235-252. World Scientific, 2002.

[114] H. Rosenberg. Some topics in minimal surface theory. Preprint.

[115] H. Rosenberg and E. Toubiana. A cylindrical type complete minimal surface in a slab of $\mathbb{R}^{3}$. Bull. Sc. Math. III, pages 241-245, 1987.

[116] H. F. Scherk. Bemerkungen über die kleinste Fläche innerhalb gegebener Grenzen. J. $R$. Angew. Math., 13:185-208, 1835.

[117] R. Schoen. Estimates for Stable Minimal Surfaces in Three Dimensional Manifolds, volume 103 of Annals of Math. Studies. Princeton University Press, 1983.

[118] R. Schoen. Uniqueness, symmetry, and embeddedness of minimal surfaces. J. of Differential Geometry, 18:791-809, 1983.

[119] M. Shiffman. On surfaces of stationary area bounded by two circles, or convex curves, in parallel planes. Annals of Math., 63:77-90, 1956.

[120] B. Solomon. On foliations of $\mathbb{R}^{n+1}$ by minimal hypersurfaces. Comm. Math. Helv., 61:67-83, 1986.

[121] M. Soret. Maximum principle at infinity for complete minimal surfaces in flat 3-manifolds. Annals of Global Analysis and Geometry, 13:101-116, 1995.

[122] M. Traizet. A balancing condition for weak limits of minimal surfaces. Preprint.

[123] M. Traizet. An embedded minimal surface with no symmetries. J. Differential Geometry, 60(1):103-153, 2002.

[124] M. Traizet and M. Weber. Hermite polynomials and helicoidal minimal surfaces. Preprint.

[125] J. Tysk. Eigenvalue estimates with applications to minimal surfaces. Pacific J. of Math., 128:361-366, 1987.

[126] M. Weber and M. Wolf. personal communication.

[127] M. Weber and M. Wolf. Teichmuller theory and handle addition for minimal surfaces. Annals of Math., 156:713-795, 2002.

[128] F. Xavier. Convex hulls of complete minimal surfaces. Math. Ann., 269:179-182, 1984.

Mathematics Department, University of Massachusetts, Amherst, MA 01003, USA

E-mail address: bill@gang.umass.edu

Universidad de Granada, Departamento de Geometria y Topologia, Fuentenueva S/n 18071 Granada, Spain

E-mail address: jperez@ugr.es 